\title{
Conformally Invariant Systems of Differential Equations and Prehomogeneous Vector Spaces of Heisenberg Parabolic Type
}

\author{
By \\ L. Barchini, Anthony C. Kable and Roger ZieraU*
}

\begin{abstract}
Several systems of partial differential operators are associated to each complex simple Lie algebra of rank greater than one. Each system is conformally invariant under the given algebra. The systems so constructed yield explicit reducibility results for a family of scalar generalized Verma modules attached to the Heisenberg parabolic subalgebra of the given Lie algebra. Points of reducibility for such families lie in the union of several arithmetic progressions, possibly overlapping. For classical algebras, enough systems are constructed to account for the first point of reducibility in each progression. The relationship between these results and a conjecture of Akihiko Gyoja is explored.
\end{abstract}

\section{$\S 1$. Introduction}

To describe our results, it is first necessary to set the scene. In the body of the paper we shall work most of the time in an exclusively algebraic framework, but it will be useful here to take a more inclusive viewpoint, mixing the analytic and the algebraic. We shall first attempt to explain the significance of our results and place them in context. To some extent, these remarks may be taken as an introduction to a broader investigation of which this work and [2] are the first fruits. Then we shall draw a map to aid the reader in navigating on the admittedly lengthy journey through the proofs.

Communicated by M. Kashiwara. Received March 19, 2007. Revised November 5, 2007. 2000 Mathematics Subject Classification(s): 22E47.

Key words: generalized Verma modules, Gyoja's conjecture, covariant maps.

The second-named author was partially supported by NSF grant DMS-0244741.

*All authors: Oklahoma State University, Stillwater, OK 74078, USA. 
Let $G$ be a split adjoint semisimple real Lie group whose complexified Lie algebra $\mathfrak{g}$ is simple and of rank greater than one. By a Heisenberg algebra we mean a two-step nilpotent Lie algebra with one-dimensional center. It is known that $\mathfrak{g}$ contains a unique conjugacy class of parabolic subalgebras whose nilradicals are Heisenberg algebras, and we call any such parabolic subalgebra a Heisenberg parabolic. Let $\mathfrak{q}$ be a Heisenberg parabolic in $\mathfrak{g}$ with nilradical $\mathfrak{n}$, $Q$ be a connected parabolic subgroup of $G$ whose complexified Lie algebra is $\mathfrak{q}$, and $\bar{Q}$ be the parabolic subgroup opposite to $Q$. Let $L$ be a Levi subgroup of $Q$. Then $\mathfrak{n}$ decomposes uniquely under the adjoint action of $L$ as $\mathfrak{n}=V^{+} \oplus Z(\mathfrak{n})$. It is known that the triple $\left(L, \mathrm{Ad}, V^{+}\right)$is a prehomogeneous vector space. Any prehomogeneous vector space constructed in this way will be said to be of Heisenberg parabolic type.

At this point, we would like to draw the reader's attention to Section 8 where we have summarized information about each of the simple complex Lie algebras and their Heisenberg parabolics. In particular, we identify the prehomogeneous vector space $\left(L, \mathrm{Ad}, V^{+}\right)$for each one. It is interesting to note that the five exceptional algebras are the simplest and most uniform from our current perspective. In contrast, each of the classical algebras displays some peculiarity or other. The reader may find it useful to refer to the data in this section as the discussion proceeds.

Having explained the meaning of the latter half of the title, we shall now address the former. Along with the Heisenberg parabolic $Q$ comes a nontrivial character $\chi$ of $L$, and we may consider the representation of $G$ smoothly parabolically induced from $\chi^{-s}$ on the parabolic $\bar{Q}$. Here $s$ is a complex parameter. If we identify the space of the induced representation with a space of smooth functions on $\mathfrak{n}$ in the usual way then we obtain a family of representations $\Pi_{s}$ of $\mathfrak{g}$. For each $Y \in \mathfrak{g}, \Pi_{s}(Y)$ is the sum of a vector field and a multiplication operator on $\mathfrak{n}$. It gives the infinitesimal action of $Y$ on $C^{\infty}(\mathfrak{n})$ via the induced representation associated to the parameter $s$. Suppose that $D_{1}, \ldots, D_{n}$ is a list of differential operators on $\mathfrak{n}$. We shall say that they constitute a conformally invariant system if, for some choice of $s$, we have identities of the form

$$
\left[\Pi_{s}(Y), D_{i}\right]=\sum_{j=1}^{n} c_{j i}^{Y} D_{j}
$$

for all $Y \in \mathfrak{g}$ and $1 \leq i \leq n$, where each $c_{j i}^{Y}$ is a smooth function on $\mathfrak{n}$. The superscript indicates the possible dependence on $Y$. The particular value of $s$ appearing in these identities will be called a special $s$ for the given system. It is possible for a given system to have more than one special $s$. 
Notice that if $D_{1}, \ldots, D_{n}$ is a conformally invariant system of differential operators then the Lie algebra $\mathfrak{g}$ acting via $\Pi_{s}$ leaves the common solution space of the system invariant. One might hope to obtain interesting representations of $\mathfrak{g}$ or, more optimistically, of $G$ on such spaces of functions. This hope provides the initial representation-theoretic motivation for considering conformally invariant systems in the context we have just described. The theory of conformally invariant systems in this sort of context was begun by Kostant in [16]. In that work, he considered the case where $n=1$; that is, where there is a single conformally invariant operator. His theory was generalized in a certain direction by Huang [10], who abandoned the framework of conformal invariance in favor of that of differential intertwining operators. We show elsewhere [2] that Kostant's original framework can also be generalized successfully. The remarks we make here on conformally invariant systems will be justified there. We note that a technical non-degeneracy assumption is a part of the definition as given in [2]. This condition is automatically satisfied in our examples and is ignored here for simplicity.

In [2], the authors relate the existence of conformally invariant systems to the existence of homomorphisms between suitable generalized Verma modules. On the other hand, the results of Collingwood and Shelton [6] relate the existence of homomorphisms between generalized Verma modules to the existence of differential intertwining operators between the corresponding smooth degenerate principal series representations. Such differential intertwining operators have been the object of intensive investigation, particularly in the case where $G$ is of Hermitian type. Jakobsen's work [11] provides some classification results for such operators. We also wish to mention that many particular examples of conformally invariant systems have appeared in the literature, without their necessarily being identified as such. One notable class of examples may be found in the work of Davidson, Enright, and Stanke [7].

There is a close connection between the existence of a conformally invariant system of differential operators with given special $s$ and the reducibility of the generalized Verma module $M(-s)=\mathfrak{U}(\mathfrak{g}) \otimes \mathfrak{u}(\mathfrak{q}) \mathbb{C}_{-s}$. Here $\mathbb{C}_{s}$ denotes the one-dimensional $\mathfrak{q}$-module obtained by letting the nilradical of $\mathfrak{q}$ act trivially on $\mathbb{C}$, and the Levi subalgebra $\mathfrak{l}$ act by $s$ times the derived character of the character $\chi$ of $L$. Indeed, with respect to a certain natural equivalence relation, equivalence classes of suitable conformally invariant systems of differential operators with a given special $s$ are in one-to-one correspondence with the irreducible $\mathfrak{l}$-submodules of the space $M(-s)^{\mathfrak{n}}$; we do not presently need to make explicit the additional conditions subsumed in the word "suitable". 
The identity operator (a differential operator of order zero) by itself forms a conformally invariant system for which every $s$ is special. The equivalence class of this system corresponds to the l-submodule of $M(-s)^{\mathfrak{n}}$ spanned by the standard generator of $M(-s)$. If, for a given $s$, there is a suitable conformally invariant system not equivalent to the system given by the identity operator then it follows that $M(-s)$ is reducible.

We have now described the prehomogeneous vector spaces of Heisenberg parabolic type and given the definition of a conformally invariant system of differential operators that is relevant in the current context. How then are these two objects to be connected? The connection is made via the classical invariant theory of the spaces $\left(L, \mathrm{Ad}, V^{+}\right)$, and we now explain this. By a covariant of $\left(L, \mathrm{Ad}, V^{+}\right)$we mean a representation $(L, \rho, W)$ of $L$ on a finitedimensional complex vector space $W$, together with an $L$-equivariant polynomial map $F: V^{+} \rightarrow W$. It is usual to speak of $F$ as being the covariant, with the target representation implicit. Some goals of classical invariant theory are to obtain a complete description of the covariants of a given space, to determine which are fundamental (in some sense to be made precise in context) and which derived, and to elucidate the algebraic and geometric meaning of the fundamental covariants. The reader may find a useful discussion of the invariant theory of the prehomogeneous vector spaces of Heisenberg parabolic type in Section 5.5 of [9].

Observe that if $F$ is a covariant then the ideal $(F) \triangleleft \mathbb{C}\left[V^{+}\right]$generated by the components of $F$ (with respect to any basis of $W$ ) is $L$-invariant. In particular, the zero set $Z(F)$ of this ideal is the union of the Zariski closures of certain $L$-orbits in $V^{+}$. In the current situation, if $\mathcal{O}$ is an $L$-orbit in $V^{+}$then its vanishing ideal $I(\mathcal{O})$ is a homogeneous ideal in $\mathbb{C}\left[V^{+}\right]$, and its homogeneous components are covariants of $V^{+}$. Note, however, that the ideal $(F)$ will not in general be radical, so that thinking in terms of covariants and $L$-invariant homogeneous ideals gives a finer perspective than thinking in terms of $L$-orbit closures in $V^{+}$.

We show that each of the spaces $\left(L, \mathrm{Ad}, V^{+}\right)$has four natural covariants, which we call $\tau_{1}, \ldots, \tau_{4}$. The subscript indicates the degree of the polynomials comprising the covariant. When $\mathfrak{g}$ is a symplectic algebra, $\tau_{3}$ and $\tau_{4}$ are identically zero; with this exception, all the $\tau_{j}$ are non-zero. Up to twists by powers of $\chi$, which we shall presently ignore, $\tau_{1}$ takes its values in the dual of $V^{+}, \tau_{2}$ takes its values in the adjoint representation of $L$ on $\mathfrak{l}, \tau_{3}$ is a self-map of $V^{+}$, and $\tau_{4}$ has one-dimensional image and is essentially a relative invariant of $V^{+}$. Thus the existence of $\tau_{1}, \ldots, \tau_{4}$ reflects the decomposition of $\mathfrak{g}$ as an $\mathfrak{l}$-module. 
This list of covariants includes some much-studied examples. For instance, if $\mathfrak{g}$ has type $\mathrm{G}_{2}$ then $V^{+}$is the space of binary cubic forms, $\tau_{2}$ is the Hessian covariant, and $\tau_{3}$ is the so-called cubocubic covariant.

Naively stated, what we do is to associate to each covariant $\tau_{j}$ a conformally invariant system $\Omega_{j}\left(Y_{1}\right), \ldots, \Omega_{j}\left(Y_{n}\right)$, where $Y_{1}, \ldots, Y_{n}$ is an ordered basis for the codomain of $\tau_{j}$. We generally refer to this conformally invariant system simply as $\Omega_{j}$. In this way, for each simple complex Lie algebra $\mathfrak{g}$, except for $\mathfrak{s l}(2)$, we produce several systems of differential operators conformally invariant under a suitable action of $\mathfrak{g}$. There are a two caveats to this naive formulation. First, in some cases the covariant $\tau_{2}$ is reducible and we actually attach a system $\Omega_{2}$ to each of its irreducible components. Secondly, we only construct $\Omega_{3}$ when $\mathfrak{g}$ is an exceptional algebra. In a sense that will be explained below, we do not require $\Omega_{3}$ for the classical algebras, so this omission is not harmful. Moreover, it is not currently clear that such a system exists for the classical algebras. The systems and their special $s$ values are summarized in Subsection 8.10 .

We must, of course, explain what we mean by saying that the system $\Omega_{j}$ is associated to the covariant $\tau_{j}$. Let $\mathbb{D}[\mathfrak{n}]$ be the Weyl algebra of $\mathfrak{n}$; that is, the ring of polynomial-coefficient differential operators on $\mathfrak{n}$. We construct a subring of $\mathbb{D}[\mathfrak{n}]$, which we shall here denote by $\mathbb{C}[\nabla]$. It arises from a right action of $N=\exp (\mathfrak{n})$ on functions on $\mathfrak{n}$, and is naturally isomorphic to $\mathcal{U}(\mathfrak{n})$. As we show in [2], every equivalence class of conformally invariant systems of differential operators on $\mathfrak{n}$ has a representative all of whose members lie in the subring $\mathbb{C}[\nabla]$. (For the present purpose, it is enough to observe that each of the differential operators $\Omega_{j}(Y)$ lies in $\left.\mathbb{C}[\nabla]\right)$. Suppose then that we have a conformally invariant system $D=D_{1}, \ldots, D_{n}$ whose members lie in $\mathbb{C}[\nabla]$. We may regard each $D_{j}$ as an element of $\mathcal{U}(\mathfrak{n})$ and consider its image in $\operatorname{gr}(\mathcal{U}(\mathfrak{n})) \cong S(\mathfrak{n})$. There is a homomorphism $S(\mathfrak{n}) \rightarrow S\left(V^{+}\right)$that extends the projection map $\mathfrak{n} \rightarrow V^{+}$and, after applying this homomorphism, we obtain a list of elements of $S\left(V^{+}\right)$. There is an $L$-relatively invariant symplectic form on $V^{+}$, unique up to proportionality, and we may use it to identify $S\left(V^{+}\right)$with $S\left(\left(V^{+}\right)^{*}\right)=\mathbb{C}\left[V^{+}\right]$. Under this identification, we obtain a list of elements of $\mathbb{C}\left[V^{+}\right]$. Let us denote by $J(D)$ the ideal in $\mathbb{C}\left[V^{+}\right]$generated by these elements. When we say that $\Omega_{j}$ is associated to $\tau_{j}$, the precise content of this statement is that $J\left(\Omega_{j}\right)=\left(\tau_{j}\right)$. When $\tau_{2}$ is reducible, the obvious refinement of this statement holds for the systems and covariants associated to each irreducible summand. The reader will see below that the construction of $\Omega_{j}$ is guided by the requirement that $J\left(\Omega_{j}\right)=\left(\tau_{j}\right)$, which thus comes for free. The difficulty is 
in establishing the conformal invariance of the resulting system for some $s$.

Because of the connection between conformally invariant systems and reducibility of generalized Verma modules, our results can be tested against Jantzen's results [12] and against the expectations arising from a conjecture of Gyoja [8]. Recall that Jantzen [12] gave a necessary and sufficient condition for reducibility of a generalized Verma module. This condition allows us to test any particular $M(s)$ for reducibility, but does not give an explicit proper submodule when reducibility is indicated. Thus it allows us to decide that a particular $s$ is a special $s$ for some conformally invariant system, without giving a hint as to what the corresponding system might be.

We now describe a modification of Gyoja's conjecture as it applies in our setting. For Gyoja's original formulation, the reader is encouraged to consult [8]. For any parabolic subalgebra $\mathfrak{q}=\mathfrak{l} \oplus \mathfrak{n}$ we follow Gyoja in defining an $L$-quasi-invariant polynomial $P \in \mathbb{C}[\mathfrak{n}]$. In our cases, we always have $P=$ $y^{2}-\Delta$, where $y$ is a coordinate on the center of $\mathfrak{n}$ and $\Delta \in \mathbb{C}\left[V^{+}\right]$is a suitably normalized relative invariant. Let $b_{P}(s)$ be the Bernstein-Sato polynomial of this quasi-invariant. Gyoja's conjecture suggests that $M(s)$ is reducible if and only if $s=r+j$, where $b_{P}(r)=0$ and $j \geq 1$ is a natural number. If $s$ is special for one of our conformally invariant systems then $M(-s)$ is reducible and so we expect that $s=-r-j$, where $r$ is a root of $b_{P}(s)$ and $j \geq 1$. In order to test this, one needs to know the Bernstein-Sato polynomial $b_{P}(s)$. The determination of this polynomial is itself a non-trivial problem, which we do not completely solve. What we do is to produce a quartic polynomial $b(s)$ such that $b_{P}(s) \mid b(s)$. We conjecture that $b_{P}(s)=b(s)$; this has been verified for low rank cases and should not be out of reach in general. In hindsight, it emerges that the special $s$ for the conformally invariant systems we construct are precisely all the numbers $-r-1$, where $r$ is a root of $b(s)$. Thus our constructions make explicit the first point of reducibility in each of the arithmetic progressions expected from Gyoja's conjecture and the conjecture that $b_{P}(s)=b(s)$. We have verified, using Jantzen's result, that this point is in fact the first reducibility point in all cases. We do not include the verification in the current work, since it would add substantially to its length; we shall return to the general question in the future. The intimate relationship between the systems we construct and the roots of $b_{P}(s)$ is one striking feature of our results.

After the present work was completed, we found the article [1] of Astashkevich and Brylinski. In this work, the authors construct a number of so-called exotic differential operators on the ring of regular functions of the complex minimal nilpotent orbit in the non-symplectic classical simple algebras. Among their 
operators may be found versions of the conformally invariant systems that we shall later refer to as $\Omega_{2}\left(Z_{0}\right), \Omega_{2}^{\text {small }}$, and $\Omega_{4}$ for these algebras. Astashkevich and Brylinski were not seeking conformally invariant systems and did not consider the conformality of the systems they constructed, yet they were led from their own starting point to some of the systems that we construct herein. This convergence may serve to support the claim that conformally invariant systems of differential operators for the simple algebras are rather remarkable objects and will tend to appear in contexts not obviously connected with conformality.

Now that we have described our results in general terms, and discussed their relationship with other results and conjectures, it is time to describe the organization of the current work. Section 2 is devoted to defining the covariants $\tau_{1}, \ldots, \tau_{4}$, establishing their general properties, and proving a number of other algebraic facts that apply uniformly to all simple complex Lie algebras and their Heisenberg subalgebras. Most of these results are routine, but we wish to draw the reader's attention to the four final propositions in this section, beginning with Proposition 2.1. In this result, we define a constant $c(\mathfrak{g}, \mathcal{C})$ associated to the algebra $\mathfrak{g}$ and a component $\mathcal{C}$ of the graph obtained by taking the Dynkin diagram of $\mathfrak{g}$ and deleting those nodes joined to the highest root in the extended Dynkin diagram and the edges that touch them. This constant is defined as the constant of proportionality between two expressions, the content of Proposition 2.1 being that they are indeed proportional. It emerges that all our special $s$ can be expressed in terms of the constants $c(\mathfrak{g}, \mathcal{C})$ and $\operatorname{dim}\left(V^{+}\right)$. Thus the constants $c(\mathfrak{g}, \mathfrak{C})$ are critical for our work. The values of these constants for all $\mathfrak{g}$ and $\mathcal{C}$ are given in Section 8; the true significance of $c(\mathfrak{g}, \mathcal{C})$ perhaps remains to be uncovered. In Propositions 2.2, 2.3, and 2.4, further proportionalities are established. The constants that appear in these proportionalities all turn out to be expressible in terms of $c(\mathfrak{g}, \mathcal{C})$ and $\operatorname{dim}\left(V^{+}\right)$. The notation introduced in Section 2 is in force for the remainder of the paper.

In Section 3, we identify a simple condition on the root system of $\mathfrak{g}$ and study the further algebraic properties of those algebras that satisfy it. The condition turns out to be equivalent to the non-vanishing of the covariants $\tau_{3}$ and $\tau_{4}$, and to be satisfied for all algebras except the symplectic algebras. Among all prehomogeneous vector space of Heisenberg parabolic type, only those associated to a symplectic algebra have no non-zero relative invariants, and this is closely connected with their failure to satisfy our condition. The notation introduced in this section remains in force subsequently; in particular, the root $\delta$ continues to play a significant role.

We describe the embedding of $\mathfrak{g}$ into the Weyl algebra of $\mathfrak{n}$ in Section 4. A 
general expression for the differential operator $\Pi_{s}(Y)$ associated to an element $Y$ of $\mathfrak{g}$ is given in Proposition 4.1. It is particularly important to have a good expression for the differential operator $D_{-\gamma}^{(s)}=\Pi_{s}\left(X_{-\gamma}\right)$ associated to the root vector $X_{-\gamma}$, where $\gamma$ is the highest root of $\mathfrak{g}$, and such an expression is given in Proposition 4.2. Next we define the operators $\nabla_{\alpha}$, where $\alpha$ is a root in $\mathfrak{n}$ other than $\gamma$. Along with the partial derivative $\partial_{\gamma}$ in the direction of the center of $\mathfrak{n}$, these operators generate a copy of $\mathcal{U}(\mathfrak{n})$ inside the Weyl algebra; this is what we denoted by $\mathbb{C}[\nabla]$ above. The operators $\nabla_{\alpha}$ are the fundamental building blocks for our conformally invariant systems. We show in Proposition 4.3 that $\nabla_{\alpha}$ commutes with $\Pi_{s}(Y)$ for $Y \in \mathfrak{n}$, and that the $\nabla_{\alpha}$ span a copy of $V^{+}$under the action of $\mathfrak{l}$ via $\Pi_{s}$. The crucial result in this section is Theorem 4.1 , which gives an expression for the commutator $\left[D_{-\gamma}^{(s)}, \nabla_{\alpha}\right]$. Note that we generally suppress the dependence on $s$ in the notation; it is included here for clarity.

Before describing the contents of Sections 5 and 6, we first explain our general strategy for obtaining conformal invariance. As observed in Lemma 2.1 , the subalgebras $\mathfrak{l}$ and $\mathfrak{n}$, and the root space $\mathfrak{g}_{-\gamma}$ generate the algebra $\mathfrak{g}$. Thus, in order to establish the relation (1.1) for all $Y \in \mathfrak{g}$, it suffices to establish it for $Y \in \mathfrak{n}$, for $Y \in \mathfrak{l}$, and for $Y=X_{-\gamma}$. The elements in our conformally invariant systems lie in $\mathbb{C}[\nabla]$ and hence commute with $\Pi_{s}(Y)$ for all $Y \in \mathfrak{n}$. Moreover, it will be clear from our construction of each system that (1.1) holds for $Y \in \mathfrak{l}$, with the coefficients $c_{i j}^{Y}$ constant. This is simply another expression of the $L$-equivariance that is built into everything we do. It therefore remains to obtain a suitable formula for the commutator $\left[D_{-\gamma}^{\left(s_{0}\right)}, \Omega\left(W_{i}\right)\right]$, where $\Omega\left(W_{i}\right)$ is a member of the system under consideration and $s_{0}$ is the special $s$ for that system. In fact, we do more than this. In each case, we obtain an identity of the form

$$
\left[D_{-\gamma}^{(s)}, \Omega\left(W_{i}\right)\right]=\sum_{j=1}^{n} c_{j i} \Omega\left(W_{j}\right)+\left(s-s_{0}\right) \Upsilon_{i}
$$

valid for all $s$, where each $\Upsilon_{i}$ is itself a differential operator. This identity simultaneously reveals the value of the special $s$ and verifies the required conformality relation for the system $\Omega\left(W_{1}\right), \ldots, \Omega\left(W_{n}\right)$ when $s$ takes this special value.

Section 5 is devoted to defining the systems $\Omega_{1}$ and $\Omega_{2}$ and obtaining (1.2) for them. The main results are Theorems 5.1, 5.2, and 5.3. In Section 6 , we continue by defining $\Omega_{3}$ (for the exceptional algebras) and $\Omega_{4}$ (for all algebras) and obtaining (1.2) for them. The main results are Theorems 6.1, 6.2, and 6.3. We have a couple of further remarks on these sections. First, 
the reader should note that the symbol $X$ has a special meaning throughout Sections 5 and 6. As explained in the introduction to Section 5 , it denotes the "generic element" of the vector space $V^{+}$, and it is necessary to understand this convention in order to interpret the statements of the above-cited theorems correctly. Secondly, the computations leading up to the conformality of the system $\Omega_{3}$ are disproportionately elaborate, but the theory of the $\Omega_{4}$ system does not rely on the $\Omega_{3}$ result and may be read independently.

The brief Section 7 describes the determination of the polynomial $b(s)$ that was mentioned above. It is largely self-contained and may be read immediately after Section 2. Finally, Section 8 is a compendium of data concerning the simple complex Lie algebras, their Heisenberg parabolic subalgebras, the polynomials $b(s)$, and the systems $\Omega_{1}, \ldots, \Omega_{4}$. By inspection of the data given in this section, the reader may verify our assertion that the special $s$ for the systems we construct are precisely the numbers $-r-1$, where $r$ is any root of the polynomial $b(s)$.

\section{$\S 2 . \quad$ The Algebraic Setting}

Let $\mathfrak{g}$ be a finite-dimensional complex simple Lie algebra. Choose a Cartan subalgebra $\mathfrak{h}$ in $\mathfrak{g}$ and let $R$ be the set of roots of $\mathfrak{g}$ with respect to $\mathfrak{h}$. Fix a positive system $R^{+} \subset R$ and let $R_{0} \subset R^{+}$be the corresponding set of simple roots. Let $\mathbf{B}_{\mathfrak{g}}$ be a positive multiple of the Killing form of $\mathfrak{g}$ and denote by $(\cdot, \cdot)$ the corresponding inner product induced on $\mathfrak{h}^{*}$. We shall specify a normalization of $\mathbf{B}_{\mathfrak{g}}$ below. For $\alpha \in R$ we denote by $\mathfrak{g}_{\alpha}$ the root space of $\mathfrak{g}$ corresponding to $\alpha$. If $U$ is any ad(h)-invariant subspace of $\mathfrak{g}$ then we denote by $R(U)$ the subset of $R$ consisting of those roots $\alpha$ such that $\mathfrak{g}_{\alpha} \subset U$.

If $\alpha, \beta \in R$ then define

$$
\begin{aligned}
& p_{\alpha, \beta}=\max \{j \in \mathbb{N} \mid \beta-j \alpha \in R\}, \\
& q_{\alpha, \beta}=\max \{j \in \mathbb{N} \mid \beta+j \alpha \in R\} .
\end{aligned}
$$

For any $\alpha, \beta \in R$ we have

$$
\frac{2(\alpha, \beta)}{(\alpha, \alpha)}=p_{\alpha, \beta}-q_{\alpha, \beta} .
$$

By Lemma 4.1.1 of [5], if $\alpha, \beta, \alpha+\beta \in R$ then we have the relation

$$
\frac{(\alpha+\beta, \alpha+\beta)}{(\beta, \beta)}=\frac{p_{\alpha, \beta}+1}{q_{\alpha, \beta}} .
$$

It is known (see Chapter 4, Section 2 of [5] or Chapter 8, Section 4.4 of [4]) that we may find a Chevalley system in $\mathfrak{g}$. That is, we may choose $X_{\alpha} \in \mathfrak{g}_{\alpha}$ and 
$H_{\alpha} \in \mathfrak{h}$ for each $\alpha \in R$ in such a way that the following conditions hold. The reader should note that we are following the normalizations of [5], although our notation is closer to that used in [4].

(C1) For each $\alpha \in R, X_{\alpha}, H_{\alpha}, X_{-\alpha}$ is an $\mathfrak{s l}(2)$-triple; in particular, $\left[X_{\alpha}, X_{-\alpha}\right]=$ $H_{\alpha}$.

(C2) For each $\alpha, \beta \in R,\left[H_{\alpha}, X_{\beta}\right]=\beta\left(H_{\alpha}\right) X_{\beta}$.

(C3) The inner product $(\cdot, \cdot)$ is positive-definite on the real span of $\left\{H_{\alpha} \mid \alpha \in\right.$ $R\}$.

(C4) For $\alpha \in R$ we have $\mathbf{B}_{\mathfrak{g}}\left(X_{\alpha}, X_{-\alpha}\right)=2 /(\alpha, \alpha)$.

(C5) For $\alpha, \beta \in R$ we have $\beta\left(H_{\alpha}\right)=2(\beta, \alpha) /(\alpha, \alpha)$.

(C6) If $\alpha, \beta, \alpha+\beta \in R$ then there is a non-zero integer $N_{\alpha, \beta}$ such that $\left[X_{\alpha}, X_{\beta}\right]=$ $N_{\alpha, \beta} X_{\alpha+\beta}$.

(C7) If $\alpha, \beta, \alpha+\beta \in R$ then $N_{\alpha, \beta} N_{-\alpha,-\beta}=-\left(p_{\alpha, \beta}+1\right)^{2}$.

(C8) If $\alpha_{1}, \alpha_{2}, \alpha_{3} \in R$ and $\alpha_{1}+\alpha_{2}+\alpha_{3}=0$ then

$$
\frac{N_{\alpha_{1}, \alpha_{2}}}{\left(\alpha_{3}, \alpha_{3}\right)}=\frac{N_{\alpha_{2}, \alpha_{3}}}{\left(\alpha_{1}, \alpha_{1}\right)}=\frac{N_{\alpha_{3}, \alpha_{1}}}{\left(\alpha_{2}, \alpha_{2}\right)} \text {. }
$$

(C9) The linear map $\omega: \mathfrak{g} \rightarrow \mathfrak{g}$ that satisfies $\omega(H)=-H$ for all $H \in \mathfrak{h}$ and $\omega\left(X_{\alpha}\right)=-X_{-\alpha}$ for all $\alpha \in R$ is an automorphism of $\mathfrak{g}$.

It will be convenient to extend the notation by defining $N_{\alpha, \beta}=0$ if $\alpha+\beta \notin R$. Note that (C9) implies that $N_{-\alpha,-\beta}=-N_{\alpha, \beta}$ for all $\alpha, \beta \in R$.

We shall call $\omega$ the Weyl automorphism of $\mathfrak{g}$; note that its square is the identity. The only freedom that remains in the choice of the $X_{\alpha}$ once all these conditions are in place is that we may multiply both $X_{\alpha}$ and $X_{-\alpha}$ by -1 for any $\alpha \in R^{+}$. Later on, we shall exploit this freedom to normalize the structure constants still further. Denote by $\gamma$ the highest root in $R$. We fix a choice of $\mathbf{B}_{\mathfrak{g}}$ by requiring that $\mathbf{B}_{\mathfrak{g}}\left(X_{\gamma}, X_{-\gamma}\right)=1$. By condition (C4), this is equivalent to requiring that $(\gamma, \gamma)=2$.

Assume now that the rank of $\mathfrak{g}$ is greater than one. Let $\mathfrak{q} \subset \mathfrak{g}$ be the standard parabolic subalgebra corresponding to the subset $\left\{\alpha \in R_{0} \mid(\alpha, \gamma)=\right.$ $0\}$ of $R_{0}$. Denote by $\mathfrak{l}$ the standard Levi subalgebra of $\mathfrak{q}$ and by $\mathfrak{n}$ the radical of $\mathfrak{q}$. Recall that $\mathfrak{n}$ is a Heisenberg algebra; that is, a two-step nilpotent algebra with one-dimensional center. In fact, $\mathfrak{z}(\mathfrak{n})=\mathfrak{g}_{\gamma}$ and $\mathfrak{n}$ has a unique ad(l)invariant subspace $V^{+}$such that $\mathfrak{n}=V^{+} \oplus \mathfrak{g}_{\gamma}$. Let $G=\operatorname{Aut}(\mathfrak{g})^{\circ}$ and $L$ be the 
connected subgroup of $G$ corresponding to the subalgebra l. The space $V^{+}$is stable under the adjoint action of $L$ and, by Vinberg's Theorem (Theorem 10.19 of [15]), the triple $\left(L, \mathrm{Ad}, V^{+}\right)$is a prehomogeneous vector space. Since $\mathfrak{g}_{\gamma}$ is one-dimensional, there is a character $\chi: L \rightarrow \mathbb{C}^{\times}$such that $\operatorname{Ad}(l) Y=\chi(l) Y$ for all $l \in L$ and $Y \in \mathfrak{g}_{\gamma}$. Note that, because $\mathbf{B}_{\mathfrak{g}}$ provides a non-zero $L$-invariant pairing between $\mathfrak{g}_{\gamma}$ and $\mathfrak{g}_{-\gamma}, \operatorname{Ad}(l) Y=\chi(l)^{-1} Y$ for all $l \in L$ and $Y \in \mathfrak{g}_{-\gamma}$.

Lemma 2.1. The algebra $\mathfrak{g}$ is generated by $\mathfrak{l}, \mathfrak{n}$, and $\mathfrak{g}_{-\gamma}$.

Proof. Let $\mathfrak{r}$ be the algebra generated by $\mathfrak{l}, \mathfrak{n}$, and $\mathfrak{g}_{-\gamma}$. Then $\mathfrak{r} \supset \mathfrak{q}$ and so $\mathfrak{r}$ is itself a parabolic subalgebra of $\mathfrak{g}$. It is strictly larger than $\mathfrak{q}$ because $\mathfrak{g}_{-\gamma} \not \subset \mathfrak{q}$. If $\mathfrak{g}$ is not of type $A_{r}$ then $\mathfrak{q}$ is a maximal parabolic subalgebra, and it follows that $\mathfrak{r}=\mathfrak{g}$. If $\mathfrak{g}=\mathfrak{s l}(n)(n \geq 3)$ then one verifies directly that $\mathfrak{r}=\mathfrak{g}$.

Let $\overline{\mathfrak{n}}$ be the radical of the parabolic opposite to $\mathfrak{q}$ so that $\mathfrak{g}=\overline{\mathfrak{n}} \oplus \mathfrak{l} \oplus \mathfrak{n}$. Then $\overline{\mathfrak{n}}$ is also a Heisenberg algebra and we have a unique decomposition $\overline{\mathfrak{n}}=V^{-} \oplus \mathfrak{g}_{-\gamma}$ with $V^{-}$an $\operatorname{ad}(\mathfrak{l})$-invariant subspace. It follows that

$$
\mathfrak{g}=\mathfrak{g}_{-\gamma} \oplus V^{-} \oplus \mathfrak{l} \oplus V^{+} \oplus \mathfrak{g}_{\gamma}
$$

is the $\operatorname{ad}\left(H_{\gamma}\right)$-weight space decomposition of $\mathfrak{g}$, where the weights, read from left to right, are $-2,-1,0,1$, and 2 . Because we have normalized $(\cdot, \cdot)$ so that $(\gamma, \gamma)=2$, we have $\left[H_{\gamma}, X_{\alpha}\right]=(\alpha, \gamma) X_{\alpha}$ for all $\alpha \in R$. Thus $(\alpha, \gamma)=1$ for all $\alpha \in R\left(V^{+}\right),(\alpha, \gamma)=0$ for all $\alpha \in R(\mathfrak{l})$, and $(\alpha, \gamma)=-1$ for all $\alpha \in R\left(V^{-}\right)$. The non-degeneracy of the bracket on $V^{+}$implies that $R\left(V^{+}\right)$is stable under the map $\alpha \mapsto \alpha^{\prime}=\gamma-\alpha$. This map is fixed-point-free because the root system $R$ is necessarily reduced. Note that $\left(\alpha^{\prime}, \alpha^{\prime}\right)=(\alpha, \alpha)$ for all $\alpha \in R\left(V^{+}\right)$. Let us write $\|\alpha\|^{2}=(\alpha, \alpha)$ for any $\alpha \in R$. It is a consequence of (C8) and the fact that $\alpha+\alpha^{\prime}+(-\gamma)=0$ that

$$
N_{\alpha,-\gamma}=-(1 / 2)\|\alpha\|^{2} N_{\alpha, \alpha^{\prime}}
$$

for all $\alpha \in R\left(V^{+}\right)$.

Lemma 2.2. For $\alpha \in R\left(V^{+}\right)$we have $N_{\alpha, \alpha^{\prime}}= \pm 2 /\|\alpha\|^{2}$.

Proof. It follows from the properties of Chevalley bases that $N_{\alpha, \alpha^{\prime}}=$ $\pm\left(p_{\alpha, \alpha^{\prime}}+1\right)$ (see [5], Section 4.2, for example). Now $\alpha^{\prime}+\alpha \in R$ and $\alpha^{\prime}+2 \alpha \notin R$ and so $q_{\alpha, \alpha^{\prime}}=1$. Therefore

$$
p_{\alpha, \alpha^{\prime}}-1=\frac{2\left(\alpha, \alpha^{\prime}\right)}{\|\alpha\|^{2}}=\frac{2-2\|\alpha\|^{2}}{\|\alpha\|^{2}},
$$


which gives $p_{\alpha, \alpha^{\prime}}+1=2 /\|\alpha\|^{2}$.

We now construct the covariant maps of $V^{+}$that will be at the heart of our work. For $0 \leq k \leq 4$ and $X \in \mathfrak{n}$, define $\tau_{k}(X) \in \mathfrak{g}$ by

$$
\tau_{k}(X)=\frac{1}{k !} \operatorname{ad}(X)^{k}\left(X_{-\gamma}\right) .
$$

We shall mostly consider $\tau_{k}(X)$ when $X \in V^{+}$; if $X$ satisfies this condition then each application of $\operatorname{ad}(X)$ increases the $H_{\gamma}$-weight by 1 and so we obtain maps

$$
\begin{aligned}
& \tau_{1}: V^{+} \rightarrow V^{-}, \\
& \tau_{2}: V^{+} \rightarrow \mathfrak{l}, \\
& \tau_{3}: V^{+} \rightarrow V^{+}, \\
& \tau_{4}: V^{+} \rightarrow \mathfrak{g}_{\gamma} .
\end{aligned}
$$

The constant map $\tau_{0}$ is defined for convenience only. Note that for $1 \leq k \leq 4$ we have

$$
\tau_{k}(X)=\frac{1}{k} \operatorname{ad}(X)\left(\tau_{k-1}(X)\right) .
$$

Lemma 2.3. For $l \in L, X \in V^{+}$, and $0 \leq k \leq 4$, we have

$$
\tau_{k}(\operatorname{Ad}(l) X)=\chi(l) \operatorname{Ad}(l) \tau_{k}(X) .
$$

Proof. For $k=0$ the claim is that $X_{-\gamma}=\chi(l) \operatorname{Ad}(l) X_{-\gamma}$ and we noted above that this is true. If $1 \leq k \leq 4$ and the equation holds of $\tau_{k-1}$ then

$$
\begin{aligned}
\tau_{k}(\operatorname{Ad}(l) X) & =\frac{1}{k} \operatorname{ad}(\operatorname{Ad}(l) X)\left(\tau_{k-1}(\operatorname{Ad}(l) X)\right) \\
& =\frac{1}{k} \operatorname{ad}(\operatorname{Ad}(l) X)\left(\chi(l) \operatorname{Ad}(l) \tau_{k-1}(X)\right) \\
& =\frac{1}{k} \chi(l) \operatorname{ad}(\operatorname{Ad}(l) X)\left(\operatorname{Ad}(l) \tau_{k-1}(X)\right) \\
& =\frac{1}{k} \chi(l) \operatorname{Ad}(l) \operatorname{ad}(X)\left(\tau_{k-1}(X)\right) \\
& =\chi(l) \operatorname{Ad}(l) \tau_{k}(X) .
\end{aligned}
$$

Since $\mathfrak{g}_{\gamma}$ is one-dimensional, there is a quartic polynomial $\Delta$ on $V^{+}$such that $\tau_{4}(X)=\Delta(X) X_{\gamma}$ for all $X \in V^{+}$. Lemma 2.3 implies that $\Delta(\operatorname{Ad}(l) X)=$ 
$\chi(l)^{2} \Delta(X)$. That is, if $\Delta$ is non-zero then it is a relatively invariant polynomial on $\left(L, \mathrm{Ad}, V^{+}\right)$associated to the character $\chi^{2}$.

It is immediate that $\left[X_{\gamma}, \tau_{j}(X)\right]=0$ for $j=3,4$. The value of this expression for $j=1,2$ is given in the next result, along with another useful commutator.

Lemma 2.4. For $X \in V^{+}$we have

$$
\begin{aligned}
& {\left[X_{\gamma}, \tau_{1}(X)\right]=-X} \\
& {\left[X_{\gamma}, \tau_{2}(X)\right]=\left[X_{-\gamma}, \tau_{2}(X)\right]=0 .}
\end{aligned}
$$

Proof. Note that

$$
\begin{aligned}
{\left[X_{\gamma}, \tau_{1}(X)\right] } & =\left[X_{\gamma},\left[X, X_{-\gamma}\right]\right] \\
& =\left[\left[X_{\gamma}, X\right], X_{-\gamma}\right]+\left[X,\left[X_{\gamma}, X_{-\gamma}\right]\right] \\
& =\left[X, H_{\gamma}\right] \\
& =-X
\end{aligned}
$$

and

$$
\begin{aligned}
{\left[X_{\gamma}, \tau_{2}(X)\right] } & =\frac{1}{2}\left[X_{\gamma},\left[X, \tau_{1}(X)\right]\right] \\
& =\frac{1}{2}\left[\left[X_{\gamma}, X\right], \tau_{1}(X)\right]+\frac{1}{2}\left[X,\left[X_{\gamma}, \tau_{1}(X)\right]\right] \\
& =-\frac{1}{2}[X, X] \\
& =0
\end{aligned}
$$

A very similar computation shows that

$$
\left[X_{-\gamma}, \tau_{2}(X)\right]=-(1 / 2)\left[\tau_{1}(X), \tau_{1}(X)\right],
$$

which is also equal to 0 .

Lemma 2.5. For $X \in V^{+}$and a scalar $y$, we have

$$
\begin{aligned}
& \tau_{1}\left(X+y X_{\gamma}\right)=\tau_{1}(X)+y H_{\gamma} \\
& \tau_{2}\left(X+y X_{\gamma}\right)=\tau_{2}(X)-y X-y^{2} X_{\gamma} \\
& \tau_{3}\left(X+y X_{\gamma}\right)=\tau_{3}(X) \\
& \tau_{4}\left(X+y X_{\gamma}\right)=\tau_{4}(X) .
\end{aligned}
$$


Proof. The first identity is immediate from the definition. From Lemma 2.4 and the first identity it follows that

$$
\begin{aligned}
\tau_{2}\left(X+y X_{\gamma}\right) & =\frac{1}{2}\left[X+y X_{\gamma}, \tau_{1}(X)+y H_{\gamma}\right] \\
& =\tau_{2}(X)-\frac{1}{2} y X+\frac{1}{2} y\left[X_{\gamma}, \tau_{1}(X)\right]-y^{2} X_{\gamma} \\
& =\tau_{2}(X)-y X-y^{2} X_{\gamma} .
\end{aligned}
$$

From Lemma 2.4 again,

$$
\begin{aligned}
\tau_{3}\left(X+y X_{\gamma}\right) & =\frac{1}{3}\left[X+y X_{\gamma}, \tau_{2}(X)-y X-y^{2} X_{\gamma}\right] \\
& =\tau_{3}(X)+\frac{1}{3} y\left[X_{\gamma}, \tau_{2}(X)\right] \\
& =\tau_{3}(X) .
\end{aligned}
$$

The last equation is immediate from this.

Lemma 2.5 may be given a more memorable form by using it to write an expression for $\operatorname{Ad}\left(\exp \left(X+y X_{\gamma}\right)\right)\left(X_{-\gamma}\right)$ as a sum of terms, one in each weight space for $\operatorname{ad}\left(H_{\gamma}\right)$. The result is that, for $X \in V^{+}$and a scalar $y$,

$$
\begin{aligned}
& \operatorname{Ad}\left(\exp \left(X+y X_{\gamma}\right)\right)\left(X_{-\gamma}\right)= \\
& \quad X_{-\gamma}+\tau_{1}(X)+\left(\tau_{2}(X)+y H_{\gamma}\right)+\left(\tau_{3}(X)-y X\right)+\left(\Delta(X)-y^{2}\right) X_{\gamma} .
\end{aligned}
$$

Lemma 2.6. For all $X \in V^{+}$we have

$$
\Delta(X)=-\frac{1}{4} \mathbf{B}_{\mathfrak{g}}\left(\tau_{1}(X), \tau_{3}(X)\right)
$$

and

$$
\Delta(X)=\frac{1}{6} \mathbf{B}_{\mathfrak{g}}\left(\tau_{2}(X), \tau_{2}(X)\right)
$$

Proof. We have

$$
\begin{aligned}
\Delta(X) & =\Delta(X) \mathbf{B}_{\mathfrak{g}}\left(X_{-\gamma}, X_{\gamma}\right) \\
& =\mathbf{B}_{\mathfrak{g}}\left(X_{-\gamma}, \tau_{4}(X)\right) \\
& =\frac{1}{4} \mathbf{B}_{\mathfrak{g}}\left(X_{-\gamma}, \operatorname{ad}(X)\left(\tau_{3}(X)\right)\right) \\
& =-\frac{1}{4} \mathbf{B}_{\mathfrak{g}}\left(\operatorname{ad}(X)\left(X_{-\gamma}\right), \tau_{3}(X)\right) \\
& =-\frac{1}{4} \mathbf{B}_{\mathfrak{g}}\left(\tau_{1}(X), \tau_{3}(X)\right) .
\end{aligned}
$$


A similar computation, taking the first identity as its starting point, yields the second identity.

Let $P\left(X+y X_{\gamma}\right)=y^{2}-\Delta(X)$. Inspired by Gyoja [8], we shall refer to $P \in \mathbb{C}[\mathfrak{n}]$ as the quasi-invariant of $\mathfrak{n}$. Note that $P(\operatorname{Ad}(l) Y)=\chi^{2}(l) P(Y)$ for all $Y \in \mathfrak{n}$ and $l \in L$, so that $P$ is indeed a quasi-invariant. Also, from (2.2),

$$
P(Y)=-\mathbf{B}_{\mathfrak{g}}\left(\operatorname{Ad}(\exp (Y))\left(X_{-\gamma}\right), X_{-\gamma}\right),
$$

which serves to distinguish $P$ in the two-dimensional space of polynomials on $\mathfrak{n}$ that share the same transformation law under $L$. We do not refer to $P$ as a relatively invariant polynomial, because we wish to reserve this term for suitable polynomials on prehomogeneous vector spaces.

We now study the $L$ action on $V^{+}$in greater detail. In particular, we shall obtain several identities that will be needed later, as well as introducing some further notation. For $X_{1}, X_{2} \in \mathfrak{n}$ we define $\left\langle X_{1}, X_{2}\right\rangle$ by

$$
\left[X_{1}, X_{2}\right]=\left\langle X_{1}, X_{2}\right\rangle X_{\gamma}
$$

Note that $\langle\cdot, \cdot\rangle$ is a degenerate alternating form on $\mathfrak{n}$. Its kernel is $\mathfrak{g}_{\gamma}$ and so its restriction to $V^{+}$is non-degenerate. We have

$$
\left\langle\operatorname{Ad}(l) X_{1}, \operatorname{Ad}(l) X_{2}\right\rangle=\chi(l)\left\langle X_{1}, X_{2}\right\rangle
$$

for all $l \in L$ and $X_{1}, X_{2} \in \mathfrak{n}$. For $\alpha \in R\left(V^{+}\right)$, we define $X_{\alpha}^{*} \in V^{+}$by $X_{\alpha}^{*}=$ $N_{\alpha, \alpha^{\prime}}^{-1} X_{\alpha^{\prime}}$. The characteristic property of these elements is that $\left\langle X_{\alpha}, X_{\beta}^{*}\right\rangle=$ $\kappa_{\alpha, \beta}$ for all $\alpha, \beta \in R\left(V^{+}\right)$; here we are using $\kappa_{\alpha, \beta}$ for the so-called Kronecker delta. In addition, we let $\left\{\xi_{\alpha}\right\}_{\alpha \in R\left(V^{+}\right)} \subset\left(V^{+}\right)^{*}$ be the dual basis to the basis $\left\{X_{\alpha}\right\}_{\alpha \in R\left(V^{+}\right)} \subset V^{+}$.

Define the functional $\lambda_{\chi}: \mathfrak{l} \rightarrow \mathbb{C}$ to be the derivative of $\chi$; that is,

$$
\lambda_{\chi}(Z)=\left.\frac{d}{d t}\right|_{t=0} \chi(\exp (t Z))
$$

By substituting $l=\exp (t Z)$ into the expression $\operatorname{Ad}(l) X_{\gamma}=\chi(l) X_{\gamma}$, differentiating, and setting $t=0$, we obtain $\left[Z, X_{\gamma}\right]=\lambda_{\chi}(Z) X_{\gamma}$.

For $l \in L, Z \in \mathfrak{l}$ and $\alpha \in R\left(V^{+}\right)$, define scalars $m_{\alpha \mu}(l)$ and $M_{\alpha \mu}(Z)$ by

$$
\operatorname{Ad}(l) X_{\alpha}=\sum_{\mu \in R\left(V^{+}\right)} m_{\alpha \mu}(l) X_{\mu}
$$

and

$$
\left[Z, X_{\alpha}\right]=\sum_{\mu \in R\left(V^{+}\right)} M_{\alpha \mu}(Z) X_{\mu}
$$


Let $m(l)=\left[m_{\alpha \beta}(l)\right]$ and $M(Z)=\left[M_{\alpha \beta}(Z)\right]$ be the matrices with these matrix coefficients as entries. The numbers $M_{\alpha \beta}(Z)$ may be expressed in terms of the structure constants of $\mathfrak{g}$, but it will be convenient to have this general notation available in addition. It is an immediate consequence of the definitions that

$$
M(\operatorname{Ad}(l) Z)=m\left(l^{-1}\right) M(Z) m(l)
$$

for all $l \in L$ and $Z \in \mathfrak{l}$. This in turn implies that

$$
M([Z, W])=M(W) M(Z)-M(Z) M(W)
$$

for all $Z, W \in \mathfrak{l}$; that is, $M$ provides a representation of the algebra $\mathfrak{l}^{\text {op }}$.

Lemma 2.7. Let $\alpha, \beta \in R\left(V^{+}\right), l \in L$ and $Z \in \mathfrak{l}$. Then

$$
\chi(l) N_{\alpha, \alpha^{\prime}} m_{\beta \alpha^{\prime}}\left(l^{-1}\right)=-N_{\beta, \beta^{\prime}} m_{\alpha \beta^{\prime}}(l)
$$

and

$$
N_{\alpha, \alpha^{\prime}} M_{\beta \alpha^{\prime}}(Z)-N_{\beta, \beta^{\prime}} M_{\alpha \beta^{\prime}}(Z)=\kappa_{\alpha \beta^{\prime}} N_{\alpha, \beta} \lambda_{\chi}(Z) .
$$

Proof. We have

$$
\chi(l)\left\langle X_{\alpha}, \operatorname{Ad}\left(l^{-1}\right) X_{\beta}\right\rangle=\left\langle\operatorname{Ad}(l) X_{\alpha}, X_{\beta}\right\rangle
$$

and the first identity follows. The second is obtained by differentiating the first.

Lemma 2.8. For all $Z \in \mathfrak{l}$ we have

$$
\sum_{\alpha \in R\left(V^{+}\right)} M_{\alpha \alpha}(Z)=(1 / 2) \operatorname{dim}\left(V^{+}\right) \lambda_{\chi}(Z) .
$$

Proof. Taking $\alpha=\beta^{\prime}$ in Lemma 2.7 gives $\lambda_{\chi}(Z)=M_{\alpha \alpha}(Z)+M_{\alpha^{\prime} \alpha^{\prime}}(Z)$ for all $Z \in \mathfrak{l}$ and $\alpha \in R\left(V^{+}\right)$. By summing over $\alpha$ we obtain the stated relation.

If $W$ is a finite-dimensional vector space and $F: V^{+} \rightarrow W$ is a map then we define

$$
\left(\partial_{\alpha} F\right)(X)=\lim _{t \rightarrow 0}\left(F\left(X+t X_{\alpha}\right)-F(X)\right) / t .
$$

We shall sometimes write $F_{\alpha}$ for $\partial_{\alpha} F$. For higher derivatives, we shall write $\partial_{\alpha \beta} F$ or $F_{\alpha \beta}$ for $\partial_{\alpha}\left(\partial_{\beta} F\right)$ and so on. 
Lemma 2.9. Let $\alpha, \beta \in R\left(V^{+}\right)$and $l \in L$. Then, for all $X \in V^{+}$,

$$
\Delta_{\alpha}(\operatorname{Ad}(l) X)=\chi(l)^{2} \sum_{\mu \in R\left(V^{+}\right)} m_{\alpha \mu}\left(l^{-1}\right) \Delta_{\mu}(X)
$$

and

$$
\Delta_{\alpha \beta}(\operatorname{Ad}(l) X)=\chi(l)^{2} \sum_{\mu, \nu \in R\left(V^{+}\right)} m_{\alpha \mu}\left(l^{-1}\right) m_{\beta \nu}\left(l^{-1}\right) \Delta_{\mu \nu}(X) .
$$

Proof. The formulas follow on differentiating the identity $\Delta(\operatorname{Ad}(l) X)=$ $\chi(l)^{2} \Delta(X)$.

For $l \in L, Z \in \mathfrak{l}$ and $\alpha, \mu \in R\left(V^{+}\right)$, we define matrix coefficients $m_{\alpha \mu}^{-}(l)$ and $M_{\alpha \mu}^{-}(Z)$ by

$$
\operatorname{Ad}(l) X_{-\alpha}=\sum_{\mu \in R\left(V^{+}\right)} m_{\alpha \mu}^{-}(l) X_{-\mu}
$$

and

$$
\left[Z, X_{-\alpha}\right]=\sum_{\mu \in R\left(V^{+}\right)} M_{\alpha \mu}^{-}(Z) X_{-\mu} .
$$

Lemma 2.10. For $l \in L, Z \in \mathfrak{l}$ and $\alpha, \beta \in R\left(V^{+}\right)$we have

$$
m_{\alpha \beta}^{-}(l)=\left(\|\beta\|^{2} /\|\alpha\|^{2}\right) m_{\beta \alpha}\left(l^{-1}\right)
$$

and

$$
M_{\alpha \beta}^{-}(Z)=-\left(\|\beta\|^{2} /\|\alpha\|^{2}\right) M_{\beta \alpha}(Z)
$$

Proof. We take the $\mathbf{B}_{\mathfrak{g}}$-inner product with $X_{\beta}$ on both sides of the equation defining $m_{\alpha \mu}^{-}(l)$ and use the $\operatorname{Ad}(l)$ invariance of $\mathbf{B}_{\mathfrak{g}}$. The first equation follows. The second is obtained by differentiating the first.

Let $\mathcal{D}(\mathfrak{g}, \mathfrak{h})$ be the Dynkin graph of $\mathfrak{g}$ with respect to $\mathfrak{h}$ and denote by $\mathcal{D}_{\gamma}(\mathfrak{g}, \mathfrak{h})$ the subgraph of $\mathcal{D}(\mathfrak{g}, \mathfrak{h})$ obtained by deleting from $\mathcal{D}(\mathfrak{g}, \mathfrak{h})$ those nodes that are joined to $-\gamma$ in the extended Dynkin diagram and the edges that involve them. The graph $\mathcal{D}_{\gamma}(\mathfrak{g}, \mathfrak{h})$ is connected except when $\mathfrak{g}$ is of type $\mathrm{B}_{r}$ or $\mathrm{D}_{r}$; in these cases, $\mathcal{D}_{\gamma}(\mathfrak{g}, \mathfrak{h})$ has two or three components, with three occurring only for $\mathrm{D}_{4}$. Let $\mathcal{C}$ be a component of $\mathcal{D}_{\gamma}(\mathfrak{g}, \mathfrak{h})$ and let $R(\mathfrak{l}, \mathcal{C})$ be the subset of $R(\mathfrak{l})$ containing those roots that are linear combinations of simple roots whose nodes lie in $\mathcal{C}$. The sets $R(\mathfrak{l}, \mathcal{C})$ form a partition of $R(\mathfrak{l})$. 
Proposition 2.1. $\quad$ Let $\mathcal{C}$ be a component of $\mathcal{D}_{\gamma}(\mathfrak{g}, \mathfrak{h})$. Then there is a constant $c(\mathfrak{g}, \mathcal{C})$ such that

$$
\sum_{\beta \in R\left(V^{+}\right)}(\alpha, \beta)(\beta, \lambda)=c(\mathfrak{g}, \mathcal{C})(\alpha, \lambda)
$$

for all $\alpha \in R\left(V^{+}\right)$and $\lambda \in R(\mathfrak{l}, \mathcal{C})$.

Proof. In the real vector space $\mathfrak{h}^{*}(\mathbb{R})$, define

$$
E=\left\{\sum_{\beta \in R\left(V^{+}\right)} a_{\beta} \beta \mid a_{\beta} \in \mathbb{R}, \sum_{\beta \in R\left(V^{+}\right)} a_{\beta}=1\right\}
$$

and

$$
v_{0}=\frac{1}{\left|R\left(V^{+}\right)\right|} \sum_{\beta \in R\left(V^{+}\right)} \beta \in E .
$$

By pairing $\beta$ and $\beta^{\prime}$ in the sum defining $v_{0}$, one sees that $v_{0}=(1 / 2) \gamma$. Thus $\left(v_{0}, v\right)=1 / 2$ for all $v \in E$ and $\left(v_{0}, \lambda\right)=0$ for all $\lambda \in R(\mathfrak{l})$. Define operations $\square$ and $\boxplus$ by $r \boxminus v=r v+(1-r) v_{0}$ and $v_{1} \boxplus v_{2}=v_{1}+v_{2}-v_{0}$ for $r \in \mathbb{R}$ and $v, v_{1}, v_{2} \in E$. With these operations, $E$ is a real vector space with zero element $v_{0}$. Choose $\lambda \in R(\mathfrak{l})$ and define $f_{1}, f_{2}: E \rightarrow \mathbb{R}$ by $f_{1}(v)=(v, \lambda)$ and

$$
f_{2}(v)=\sum_{\beta \in R\left(V^{+}\right)}(v, \beta)(\beta, \lambda) .
$$

By making use of the equations $\left(v_{0}, \lambda\right)=0$ and $\left(v_{0}, v\right)=1 / 2$ for all $v \in E$, it is easy to check that both $f_{1}$ and $f_{2}$ are linear functionals. Moreover, $f_{1}$ is non-zero, for otherwise $H_{\lambda}$ would centralize $\mathfrak{n}$. Suppose that $v \in \operatorname{ker}\left(f_{1}\right)$ and let $s_{\lambda}$ be the reflection corresponding to $\lambda$ in the Weyl group of $R$. Note that $s_{\lambda}(\gamma)=\gamma$ and so $s_{\lambda}$ leaves the set $R\left(V^{+}\right)$stable. Since $v \in \operatorname{ker}\left(f_{1}\right), s_{\lambda}(v)=v$. Thus

$$
\begin{aligned}
f_{2}(v) & =\sum_{\beta \in R\left(V^{+}\right)}(v, \beta)(\beta, \lambda) \\
& =\sum_{\beta \in R\left(V^{+}\right)}\left(s_{\lambda}(v), \beta\right)(\beta, \lambda) \\
& =\sum_{\beta \in R\left(V^{+}\right)}\left(v, s_{\lambda}(\beta)\right)(\beta, \lambda) \\
& =\sum_{\beta \in R\left(V^{+}\right)}(v, \beta)\left(s_{\lambda}(\beta), \lambda\right)
\end{aligned}
$$




$$
\begin{aligned}
& =\sum_{\beta \in R\left(V^{+}\right)}(v, \beta)\left(\beta, s_{\lambda}(\lambda)\right) \\
& =\sum_{\beta \in R\left(V^{+}\right)}(v, \beta)(\beta,-\lambda) \\
& =-f_{2}(v),
\end{aligned}
$$

and so $f_{2}(v)=0$. That is, $\operatorname{ker}\left(f_{1}\right) \subset \operatorname{ker}\left(f_{2}\right)$, and it follows that there is a constant $c(\lambda)$ such that $f_{2}=c(\lambda) f_{1}$.

Now suppose that $\lambda_{1}, \lambda_{2} \in R(\mathfrak{l})$ are such that $\lambda_{1}+\lambda_{2} \in R(\mathfrak{l})$. By using the defining property of the constants $c(\lambda)$, one obtains the equation

$$
\left(c\left(\lambda_{1}+\lambda_{2}\right)-c\left(\lambda_{1}\right)\right) \lambda_{1}=\left(c\left(\lambda_{2}\right)-c\left(\lambda_{1}+\lambda_{2}\right)\right) \lambda_{2} .
$$

However, $\lambda_{1}$ and $\lambda_{2}$ are necessarily linearly independent and so we conclude that $c\left(\lambda_{1}\right)=c\left(\lambda_{2}\right)=c\left(\lambda_{1}+\lambda_{2}\right)$. A downward induction on the height of a root in $R(\mathfrak{l}, \mathrm{e})$ now suffices to show that the function $\lambda \mapsto c(\lambda)$ is constant on each $R(\mathfrak{l}, \mathcal{C})$. This is equivalent to our claim.

If $\mathcal{C}$ is a component of $\mathcal{D}_{\gamma}(\mathfrak{g}, \mathfrak{h})$ then we let $\mathfrak{l}(\mathfrak{C})$ denote the ideal of $\mathfrak{l}$ generated by the set $\left\{X_{\lambda} \mid \lambda \in R(\mathfrak{l}, \mathcal{C})\right\}$. In every case, $\mathfrak{l}(\mathcal{C})$ is a simple complex Lie algebra. In particular, $\mathfrak{l}(\mathcal{C}) \subset \operatorname{ker}\left(\lambda_{\chi}\right)$ for all $\mathfrak{C}$. The ideal $[\mathfrak{l}, \mathfrak{l}]$ of $\mathfrak{l}$ is the direct sum of the $\mathfrak{l}(\mathcal{C})$ over all components. We let $\operatorname{pr}_{\mathcal{C}}: \mathfrak{l} \rightarrow \mathfrak{l}(\mathcal{C})$ be the projection map associated with this direct sum.

Lemma 2.11. Suppose that $\mathfrak{g}$ is not of type $\mathrm{A}_{r}$, and let $\mathfrak{l}_{0}=[\mathfrak{l}, \mathfrak{l}]$. Then the $\mathfrak{I}_{0}$-module $V^{+} \otimes V^{+}$is multiplicity-free.

Proof. The proof is by case-by-case consideration. We number the fundamental weights for each simple summand of $\mathfrak{l}_{0}$ as in the tables in [3]. For any $\mathfrak{l}_{0}$-module $W$, we write $\varpi(W)$ for the list of highest weights that occur in $W$. Let us first consider the exceptional algebras. The following table gives the decomposition data in these cases.

\begin{tabular}{cccc}
$\mathfrak{g}$ & $\mathfrak{l}_{0}$ & $\varpi\left(V^{+}\right)$ & $\varpi\left(V^{+} \otimes V^{+}\right)$ \\
\hline $\mathrm{E}_{6}$ & $\mathrm{~A}_{5}$ & $\varpi_{3}$ & $0, \varpi_{1}+\varpi_{5}, \varpi_{2}+\varpi_{4}, 2 \varpi_{3}$ \\
$\mathrm{E}_{7}$ & $\mathrm{D}_{6}$ & $\varpi_{6}$ & $0, \varpi_{2}, \varpi_{4}, 2 \varpi_{6}$ \\
$\mathrm{E}_{8}$ & $\mathrm{E}_{7}$ & $\varpi_{7}$ & $0, \varpi_{1}, \varpi_{6}, 2 \varpi_{7}$ \\
$\mathrm{~F}_{4}$ & $\mathrm{C}_{3}$ & $\varpi_{3}$ & $0,2 \varpi_{1}, 2 \varpi_{2}, 2 \varpi_{3}$ \\
$\mathrm{G}_{2}$ & $\mathrm{~A}_{1}$ & $3 \varpi_{1}$ & $0,2 \varpi_{1}, 4 \varpi_{1}, 6 \varpi_{1}$ \\
\hline
\end{tabular}


In each case, $V^{+} \otimes V^{+}$is visibly multiplicity-free. To aid in obtaining these facts, several observations are helpful. First, if $V^{+}$has highest weight $\varpi$ then $2 \varpi$ must occur as a highest weight of $V^{+} \otimes V^{+}$. Secondly, the trivial representation necessarily occurs precisely once, because $V^{+} \cong\left(V^{+}\right)^{*}$ as an $\mathfrak{l}_{0}$-module. Thirdly, $V^{-} \cong V^{+}$as an $\mathfrak{l}_{0}$-module, and the existence of the bracket map $[\cdot, \cdot]: V^{+} \otimes V^{-} \rightarrow \mathfrak{l}$ forces the adjoint representation of $\mathfrak{l}_{0}$ to appear at least once in $V^{+} \otimes V^{+}$. Finally, if $\alpha$ is a simple root such that $(\varpi, \alpha) \neq 0$ then $2 \varpi-\alpha$ is a highest weight of $V^{+} \otimes V^{+}$. These observations and the Weyl dimension formula are sufficient to complete the table.

The statement for the algebras of types $\mathrm{B}_{r}, \mathrm{C}_{r}$, and $\mathrm{D}_{r}$ is substantially simpler to obtain, since it requires nothing more than the decomposition of the tensor square of the standard representation of a classical algebra.

Proposition 2.2. $\quad$ There is a constant $p(\mathfrak{g}, \mathcal{C})$ associated to each component $\mathcal{C}$ of $\mathcal{D}_{\gamma}(\mathfrak{g}, \mathfrak{h})$ such that

$$
\sum_{\epsilon \in R\left(V^{+}\right)}\|\epsilon\|^{2}\left[\left[X, X_{-\epsilon}\right],\left[X_{\epsilon}, Y\right]\right]=\sum_{\mathcal{C}} p(\mathfrak{g}, \mathcal{C}) \operatorname{pr}_{\mathfrak{C}}([X, Y])
$$

for all $X \in V^{+}$and $Y \in V^{-}$.

Proof. When $\mathfrak{g}$ has type $\mathrm{A}_{r}$, the formula can be checked by direct computation, with the constant $p\left(\mathrm{~A}_{r}\right)=2(r-1)$ for the single component of $\mathcal{D}_{\gamma}(\mathfrak{g}, \mathfrak{h})$. We now assume that $\mathfrak{g}$ is not of type $\mathrm{A}_{r}$. Define $f: V^{+} \otimes V^{-} \rightarrow \mathfrak{l}$ by sending the simple tensor $X \otimes Y$ to the formula on the left-hand side of the proposed identity. It is evident that the image of $f$ lies in $[\mathfrak{l}, \mathfrak{l}]$ and, since $\mathfrak{g}$ does not have type $\mathrm{A}_{r}$, this is equal to $\sum_{\mathcal{C}} \mathfrak{l}(\mathcal{C})$. For $l \in L, X \in V^{+}$, and $Y \in V^{-}$, we have

$$
\begin{aligned}
& f(\operatorname{Ad}(l) X \otimes \operatorname{Ad}(l) Y) \\
& \quad=\sum_{\epsilon}\|\epsilon\|^{2}\left[\left[\operatorname{Ad}(l) X, X_{-\epsilon}\right],\left[X_{\epsilon}, \operatorname{Ad}(l) Y\right]\right] \\
& \quad=\operatorname{Ad}(l) \sum_{\epsilon}\|\epsilon\|^{2}\left[\left[X, \operatorname{Ad}\left(l^{-1}\right) X_{-\epsilon}\right],\left[\operatorname{Ad}\left(l^{-1}\right) X_{\epsilon}, Y\right]\right] \\
& \quad=\operatorname{Ad}(l) \sum_{\epsilon, \mu, \nu}\|\epsilon\|^{2} m_{\epsilon \mu}^{-}\left(l^{-1}\right) m_{\epsilon \nu}\left(l^{-1}\right)\left[\left[X, X_{-\mu}\right],\left[X_{\nu}, Y\right]\right] \\
& =\operatorname{Ad}(l) \sum_{\epsilon, \mu, \nu}\|\mu\|^{2} m_{\mu \epsilon}(l) m_{\epsilon \nu}\left(l^{-1}\right)\left[\left[X, X_{-\mu}\right],\left[X_{\nu}, Y\right]\right] \\
& =\operatorname{Ad}(l) \sum_{\mu, \nu}\|\mu\|^{2} m_{\mu \nu}(e)\left[\left[X, X_{-\mu}\right],\left[X_{\nu}, Y\right]\right]
\end{aligned}
$$




$$
\begin{aligned}
& =\operatorname{Ad}(l) \sum_{\mu}\|\mu\|^{2}\left[\left[X, X_{-\mu}\right],\left[X_{\mu}, Y\right]\right] \\
& =\operatorname{Ad}(l) f(X \otimes Y),
\end{aligned}
$$

where we have used Lemma 2.10 from line four to line five. That is, $f$ is an $L$-homomorphism and, consequently, an $\mathfrak{l}_{0}$-homomorphism. Since $V^{+} \otimes$ $V^{-} \cong V^{+} \otimes V^{+}$is multiplicity-free, any two homomorphisms from $V^{+} \otimes V^{-}$ to $\mathfrak{l}(\mathrm{C})$ are proportional. By applying this observation to the homomorphisms $\operatorname{pr}_{\mathcal{C}} \circ f$ and $X \otimes Y \mapsto \operatorname{pr}_{\mathcal{C}}([X, Y])$ for each component $\mathcal{C}$, we obtain the required statement.

Proposition 2.3. For $Z \in \mathfrak{l}_{0}$ we have

$$
\sum_{\alpha \in R\left(V^{+}\right)}\|\alpha\|^{2}\left[\left[Z, X_{\alpha}\right], X_{-\alpha}\right]=2 \sum_{\mathcal{C}} c(\mathfrak{g}, \mathcal{C}) \operatorname{pr}_{\mathcal{C}}(Z) .
$$

Proof. We define a map $f: \mathfrak{l} \rightarrow \mathfrak{l}$ by

$$
f(W)=\sum_{\alpha \in R\left(V^{+}\right)}\|\alpha\|^{2}\left[\left[W, X_{\alpha}\right], X_{-\alpha}\right] .
$$

One checks that $f$ is an $L$-homomorphism and hence an $\mathfrak{l}_{0}$-homomorphism. Since $\mathfrak{l}_{0}$ does not contain the trivial representation of $\mathfrak{l}_{0}$, it follows that $f\left(\mathfrak{l}_{0}\right) \subset$ $\mathfrak{l}_{0}$. The ideals $\mathfrak{l}(\mathcal{C})$ are non-isomorphic irreducible $\mathfrak{l}_{0}$-modules and it follows that there are constants $k(\mathcal{C})$ such that

$$
f(Z)=\sum_{\mathcal{C}} k(\mathcal{C}) \operatorname{pr}_{\mathfrak{C}}(Z)
$$

for all $Z \in \mathfrak{l}_{0}$. It remains to show that $k(\mathcal{C})=2 c(\mathfrak{g}, \mathcal{C})$. Let $\lambda \in R(\mathfrak{l}, \mathcal{C})$ and take $Z=H_{\lambda}$. After some simplification, we obtain

$$
\frac{2}{\|\lambda\|^{2}} \sum_{\alpha}\|\alpha\|^{2}(\lambda, \alpha) H_{\alpha}=k(\mathcal{C}) H_{\lambda} .
$$

Let $\beta \in R\left(V^{+}\right)$and apply $\beta$ to both sides of the previous equality. The result is

$$
2 \sum_{\alpha}(\lambda, \alpha)(\alpha, \beta)=k(\mathcal{C})(\lambda, \beta) .
$$

By comparing this with the result of Proposition 2.1, we obtain $2 c(\mathfrak{g}, \mathcal{C})(\lambda, \beta)=$ $k(\mathcal{C})(\lambda, \beta)$ for all $\lambda \in R(\mathfrak{l}, \mathcal{C})$ and all $\beta \in R\left(V^{+}\right)$. The fact that $H_{\lambda}$ does not centralize $\mathfrak{n}$ implies that we may find some $\beta \in R\left(V^{+}\right)$such that $(\lambda, \beta) \neq 0$ and so $k(\mathcal{C})=2 c(\mathfrak{g}, \mathcal{C})$. 
Proposition 2.4. There is a constant $q(\mathfrak{g}, \mathcal{C})$ associated to each component $\mathcal{C}$ of $\mathcal{D}_{\gamma}(\mathfrak{g}, \mathfrak{h})$ such that

$$
\sum_{\epsilon \in R\left(V^{+}\right)}\|\epsilon\|^{2}\left[\left[Z,\left[X, X_{-\epsilon}\right]\right], X_{\epsilon}\right]=\sum_{\mathcal{C}} q(\mathfrak{g}, \mathcal{C})\left[\operatorname{pr}_{\mathcal{C}}(Z), X\right]
$$

for all $X \in V^{+}$and $Z \in \mathfrak{l}$.

Proof. The formula can be checked by direct computation when $\mathfrak{g}$ is of type $\mathrm{A}_{r}$. In this case, the constant $q(\mathfrak{g}, \mathcal{C})$ has the value $2(r-1)$ for the unique component $\mathcal{C}$ of $\mathcal{D}_{\gamma}(\mathfrak{g}, \mathfrak{h})$. We now assume that $\mathfrak{g}$ is not of this type. Define $f: \mathfrak{l} \otimes V^{+} \rightarrow V^{+}$to be the linear map that sends the simple tensor $Z \otimes X$ to the left-hand side of the proposed identity. By using Lemma 2.10, one checks that $f$ is an $L$-homomorphism and so an $\mathfrak{l}_{0}$-homomorphism. Clearly $f$ vanishes on the center of $\mathfrak{l}$. By restriction, one obtains an $\mathfrak{l}_{0}$-homomorphism $\mathfrak{l}(\mathcal{C}) \otimes V^{+} \rightarrow V^{+}$. However,

$$
\operatorname{Hom}_{\mathfrak{l}_{0}}\left(\mathfrak{l}(\mathcal{C}) \otimes V^{+}, V^{+}\right) \cong \operatorname{Hom}_{\mathfrak{l}_{0}}\left(\mathfrak{l}(\mathcal{C}), V^{+} \otimes V^{+}\right) \cong \mathbb{C}
$$

by Lemma 2.11, and consequently this homomorphism is proportional to any non-zero homomorphism $\mathfrak{l}(\mathcal{C}) \otimes V^{+} \rightarrow V^{+}$. The linear map that satisfies $Z \otimes$ $X \mapsto[Z, X]$ is such a homomorphism. The claim follows.

In Section 3, we shall see that $p(\mathfrak{g}, \mathcal{C})=q(\mathfrak{g}, \mathcal{C})$, and obtain a relationship between this constant and $c(\mathfrak{g}, \mathcal{C})$.

\section{$\S 3 . \quad$ Further Algebraic Properties}

In Section 2 we did not consider the question of whether the maps $\tau_{1}, \ldots, \tau_{4}$ are non-zero on $V^{+}$, and nothing done there requires that they be so. It is easy to see that $\tau_{1}$ is always a linear isomorphism between $V^{+}$and $V^{-}$, and that $\tau_{2}$ cannot be identically zero. In contrast, $\tau_{3}$ and $\tau_{4}$ can be identically zero. In fact, this occurs if and only if $\mathfrak{g}$ is of type $\mathrm{C}_{r}$ (including $\mathrm{C}_{2}=\mathrm{B}_{2}$ ). That $\tau_{3}$ and $\tau_{4}$ are identically zero in this case may be verified directly. That they are not in any other case will follow from the discussion below.

In this section, we place an additional condition on the algebra $\mathfrak{g}$. Namely, we assume that there is a root $\delta \in R\left(V^{+}\right)$such that $\delta^{\prime}-\delta=\gamma-2 \delta \notin R$. Reference to the data presented in Section 8 will show that this condition holds for all $\mathfrak{g}$ except for those of type $\mathrm{C}_{r}$ with $r \geq 2$. In all cases, $\delta$ may be chosen to be a simple root in $R\left(V^{+}\right)$if desired. Our purpose in this section is to investigate the consequences of the existence of $\delta$. 
The $\delta$-string through $\delta^{\prime}$ is $\delta^{\prime}, \gamma$ and so $p_{\delta, \delta^{\prime}}=0$ and $q_{\delta, \delta^{\prime}}=1$. From (2.1), it follows that $(\delta, \delta)=(\gamma, \gamma)=2$. We know that $(\gamma, \delta)=1$ and so $\left(\delta, \delta^{\prime}\right)=-1$. Condition (C7) implies that $N_{\delta, \delta^{\prime}} N_{-\delta,-\delta^{\prime}}=-1$ and, since $N_{\delta, \delta^{\prime}}$ and $N_{-\delta,-\delta^{\prime}}$ are both integers, we conclude that $N_{\delta, \delta^{\prime}}= \pm 1$. Recall that we are free to multiply both $X_{\gamma}$ and $X_{-\gamma}$ by -1 if we wish. Doing so while leaving all other $X_{\alpha}$ unchanged will result in replacing $N_{\delta, \delta^{\prime}}$ by $-N_{\delta, \delta^{\prime}}$. We may thus assume that the Chevalley system has been normalized so that $N_{\delta, \delta^{\prime}}=1$. Let $\mathfrak{m}$ denote the subalgebra of $\mathfrak{g}$ generated by the set $\left\{X_{ \pm \delta}, X_{ \pm \delta^{\prime}}\right\}$.

Lemma 3.1. We have

$$
\begin{aligned}
& N_{\delta, \delta^{\prime}}=N_{-\delta, \gamma}=N_{\delta^{\prime},-\gamma}=1, \\
& N_{\delta,-\gamma}=N_{-\delta,-\delta^{\prime}}=N_{-\delta^{\prime}, \gamma}=-1 .
\end{aligned}
$$

The linear map $\mathfrak{m} \rightarrow \mathfrak{s l}(3)$ satisfying

$$
\begin{array}{lll}
H_{\delta} \mapsto E_{11}-E_{22}, & X_{\delta} \mapsto E_{12}, & X_{-\delta} \mapsto E_{21}, \\
H_{\delta^{\prime}} \mapsto E_{22}-E_{33}, & X_{\delta^{\prime}} \mapsto E_{23}, & X_{-\delta^{\prime}} \mapsto E_{32}, \\
H_{\gamma} \mapsto E_{11}-E_{33}, & X_{\gamma} \mapsto E_{13}, & X_{-\gamma} \mapsto E_{31}
\end{array}
$$

is an isomorphism of Lie algebras.

Proof. Since $\delta+\delta^{\prime}+(-\gamma)=0$, condition (C8) implies that $N_{\delta, \delta^{\prime}}, N_{\delta^{\prime},-\gamma}$, and $N_{-\gamma, \delta}$ are all equal. The constant $N_{\delta, \delta^{\prime}}$ has been normalized to be 1 and this confirms three of the six required values. We have seen that $N_{\delta, \delta^{\prime}} N_{-\delta,-\delta^{\prime}}=-1$ and so $N_{-\delta,-\delta^{\prime}}=-1$. But $(-\delta)+\left(-\delta^{\prime}\right)+\gamma=0$ and hence, by (C8) again, $N_{-\delta,-\delta^{\prime}}, N_{-\delta^{\prime}, \gamma}$, and $N_{\gamma,-\delta}$ are all equal. This confirms the other three values.

By hypothesis, $\delta-\delta^{\prime}=\gamma-2 \delta \notin R$ and so $\left[X_{\delta}, X_{-\delta^{\prime}}\right]=0$ and $\left[X_{-\delta}, X_{\delta^{\prime}}\right]=$ 0 . Since $\delta+\delta^{\prime}=\gamma$ and all three roots have the same length, $H_{\delta}+H_{\delta^{\prime}}=H_{\gamma}$. It follows that the set $\left\{X_{ \pm \delta}, X_{ \pm \delta^{\prime}}, X_{ \pm \gamma}, H_{\delta}, H_{\delta^{\prime}}\right\}$ is a basis for the algebra $\mathfrak{m}$. We now have enough information to determine all the structure constants of $\mathfrak{m}$ with respect to this basis and they match those of $\mathfrak{s l}(3)$ with respect to its standard basis. The second claim follows.

Lemma 3.2. Let $\alpha \in R\left(V^{+}\right)-\left\{\delta, \delta^{\prime}\right\}$. Then exactly one of $\alpha-\delta$ and $\alpha-\delta^{\prime}$ is a root, and $\alpha-2 \delta$ and $\alpha-2 \delta^{\prime}$ are not roots.

Proof. First note that $\alpha+\delta$ and $\alpha+\delta^{\prime}$ are not roots and so $q_{\delta, \alpha}=q_{\delta^{\prime}, \alpha}=0$. Thus

$$
p_{\delta, \alpha}+p_{\delta^{\prime}, \alpha}=\frac{2(\alpha, \delta)}{(\delta, \delta)}+\frac{2\left(\alpha, \delta^{\prime}\right)}{\left(\delta^{\prime}, \delta^{\prime}\right)}=(\alpha, \delta)+\left(\alpha, \delta^{\prime}\right)=(\alpha, \gamma)=1,
$$


from which it follows that $\left\{p_{\delta, \alpha}, p_{\delta^{\prime}, \alpha}\right\}=\{0,1\}$. This is equivalent to the required statements.

We define $S(\delta)$ by $S(\delta)=\left\{\alpha \in R\left(V^{+}\right) \mid \alpha-\delta \in R\right\}$ and $S\left(\delta^{\prime}\right)$ similarly with $\delta$ replaced by $\delta^{\prime}$. Equivalently, $S(\delta)$ consists of those $\alpha \in R\left(V^{+}\right)$such that $(\alpha, \delta)=1$ and $S\left(\delta^{\prime}\right)$ consists of those such that $(\alpha, \delta)=0$. It follows from Lemma 3.2 that $R\left(V^{+}\right)$is the disjoint union of $\left\{\delta, \delta^{\prime}\right\}, S(\delta)$ and $S\left(\delta^{\prime}\right)$. Moreover, it is clear from the definition that $S(\delta)^{\prime}=S\left(\delta^{\prime}\right)$. For later use, we note that if $\alpha, \beta \in R\left(V^{+}\right)$and $\alpha+\beta=2 \delta$ then $\alpha=\beta=\delta$. This follows on taking the inner product with $\delta$ on both sides of the equation $\alpha+\beta=2 \delta$ and using the above observations. A similar remark applies to the equation $\alpha+\beta=2 \delta^{\prime}$, which implies that $\alpha=\beta=\delta^{\prime}$.

Lemma 3.3. $\quad$ Let $\mathcal{C}$ be a component of $\mathcal{D}_{\gamma}(\mathfrak{g}, \mathfrak{h})$ and $\lambda \in R(\mathfrak{l}, \mathcal{C})$. Then

$$
(c(\mathfrak{g}, \mathcal{C})-3)(\delta, \lambda)=\left(\sum_{\beta \in S(\delta)} \beta, \lambda\right) .
$$

Proof. This follows immediately from Proposition 2.1 on taking $\alpha=\delta$ and using the definition of the set $S(\delta)$.

Proposition 3.1. Let $\mathcal{C}$ be a component of $\mathcal{D}_{\gamma}(\mathfrak{g}, \mathfrak{h})$. Then

$$
c(\mathfrak{g}, \mathcal{C})+(1 / 2) p(\mathfrak{g}, \mathcal{C})=(1 / 2)\left(\operatorname{dim}\left(V^{+}\right)+4\right) .
$$

Proof. If $\mathfrak{g}$ has type $\mathrm{A}_{r}$ then the formula may be verified directly, since in this case $p(\mathfrak{g}, \mathfrak{C})=2(r-1), c(\mathfrak{g}, \mathfrak{C})=2$, and $\operatorname{dim}\left(V^{+}\right)=2(r-1)$. We now assume that $\mathfrak{g}$ is not of type $\mathrm{A}_{r}$.

Take $X=X_{\delta}$ and $Y=X_{-\delta}$ in Proposition 2.2. After a little simplification, the left-hand side of the formula becomes

$$
\begin{aligned}
& \sum_{\epsilon \in S(\delta)}\|\epsilon\|^{2} N_{\delta,-\epsilon} N_{\epsilon,-\delta} H_{\delta-\epsilon} \\
& =\sum_{\epsilon \in S(\delta)}\|\epsilon\|^{2}\left(p_{\delta,-\epsilon}+1\right)^{2} H_{\delta-\epsilon} \\
& =\sum_{\epsilon \in S(\delta)}\|\epsilon\|^{2} H_{\delta-\epsilon},
\end{aligned}
$$

because $p_{\delta,-\epsilon}=0$. On the other hand, $\left[X_{\delta}, X_{-\delta}\right]=H_{\delta}$ and so

$$
\sum_{\epsilon \in S(\delta)}\|\epsilon\|^{2} H_{\delta-\epsilon}=\sum_{\mathcal{C}^{\prime}} p\left(\mathfrak{g}, \mathcal{C}^{\prime}\right) \operatorname{pr}_{\mathcal{C}^{\prime}}\left(H_{\delta}\right)
$$


where the sum is over all components of $\mathcal{D}_{\gamma}(\mathfrak{g}, \mathfrak{h})$. This formula is valid for any choice of $\delta$. Let us assume now that $\delta$ has been chosen to be a simple root, which is always possible, and then choose a simple root $\lambda$ in the component $\mathcal{C}$ such that $(\lambda, \delta) \neq 0$. We evaluate $\lambda$ on both sides of (3.1). Note that $s_{\delta}(\epsilon)=\epsilon-\delta$ and so $\|\epsilon-\delta\|=\|\epsilon\|$. Armed with this observation, the value of $\lambda$ on the left-hand side of (3.1) simplifies to

$$
\begin{aligned}
2 \sum_{\epsilon \in S(\delta)}(\lambda, \delta-\epsilon) & =2|S(\delta)|(\lambda, \delta)-2 \sum_{\epsilon \in S(\delta)}(\lambda, \epsilon) \\
& =2|S(\delta)|(\lambda, \delta)-2(c(\mathfrak{g}, \mathcal{C})-3)(\lambda, \delta),
\end{aligned}
$$

where the last equality follows from Lemma 3.3. Since $\mathfrak{g}$ is not of type $\mathrm{A}_{r}$,

$$
\sum_{\mathcal{C}^{\prime}} \operatorname{pr}_{\mathcal{C}^{\prime}}\left(H_{\delta}\right)=H_{\delta}-(1 / 2) H_{\gamma} .
$$

The root $\lambda$ vanishes identically on $\mathfrak{l}\left(\mathfrak{C}^{\prime}\right) \cap \mathfrak{h}$ with $\mathfrak{C}^{\prime} \neq \mathcal{C}$ and $\lambda\left(H_{\gamma}\right)=0$. Thus

$$
\lambda\left(\operatorname{pr}_{\mathfrak{C}}\left(H_{\delta}\right)\right)=\lambda\left(H_{\delta}\right)=(\lambda, \delta) .
$$

By using this equation, the value of $\lambda$ on the right-hand side of (3.1) is found to be $p(\mathfrak{g}, \mathcal{C})(\lambda, \delta)$. Since $(\lambda, \delta) \neq 0$, we conclude that

$$
p(\mathfrak{g}, \mathcal{C})=2|S(\delta)|-2 c(\mathfrak{g}, \mathcal{C})+6 .
$$

Finally, $|S(\delta)|=(1 / 2)\left(\operatorname{dim}\left(V^{+}\right)-2\right)$ and the claim follows.

By using Lemma 3.3 , it is easy to find $c(\mathfrak{g}, \mathcal{C})$ in all cases but type $\mathrm{C}_{r}$, to which the lemma does not apply. In the remaining case, $c(\mathfrak{g}, \mathcal{C})$ may be found directly. Once $c(\mathfrak{g}, \mathcal{C})$ is known, the value of $p(\mathfrak{g}, \mathcal{C})$ follows from Proposition 3.1 , again excepting type $\mathrm{C}_{r}$.

Proposition 3.2. $\quad$ Let $\mathcal{C}$ be a component of $\mathcal{D}_{\gamma}(\mathfrak{g}, \mathfrak{h})$. Then

$$
c(\mathfrak{g}, \mathcal{C})+(1 / 2) q(\mathfrak{g}, \mathcal{C})=(1 / 2)\left(\operatorname{dim}\left(V^{+}\right)+4\right) .
$$

In particular, $p(\mathfrak{g}, \mathcal{C})=q(\mathfrak{g}, \mathcal{C})$.

Proof. If $\mathfrak{g}$ is of type $\mathrm{A}_{r}$ then the formula follows from the fact that $q(\mathfrak{g}, \mathcal{C})=2(r-1)$ in this case. We now suppose that $\mathfrak{g}$ is not of this type. Let us choose $\delta$ to be a simple root and $\lambda \in R(\mathfrak{l}, \mathcal{C})$ to be a simple root such that $(\delta, \lambda) \neq 0$. In Proposition 2.4 we take $Z=H_{\lambda}$ and $X=X_{\delta}$ to obtain

$$
\sum_{\epsilon \in R\left(V^{+}\right)}\|\epsilon\|^{2}\left[\left[H_{\lambda},\left[X_{\delta}, X_{-\epsilon}\right]\right], X_{\epsilon}\right]=q(\mathfrak{g}, \mathcal{C})\left[H_{\lambda}, X_{\delta}\right] .
$$


The right-hand side is equal to $2 q(\mathfrak{g}, \mathcal{C})\left((\lambda, \delta) /\|\lambda\|^{2}\right) X_{\delta}$. The left-hand side evaluates to

$$
\frac{2}{\|\lambda\|^{2}} \sum_{\epsilon \in S(\delta)}\|\epsilon\|^{2} N_{\delta,-\epsilon} N_{\delta-\epsilon, \epsilon}(\lambda, \delta-\epsilon) X_{\delta} .
$$

Now $N_{\delta-\epsilon, \epsilon} /\|\delta\|^{2}=N_{\delta,-\epsilon} /\|\epsilon\|^{2}, N_{\delta,-\epsilon}^{2}=1$, and $\|\delta\|^{2}=2$, and so the previous expression is equal to

$$
\frac{4}{\|\lambda\|^{2}} \sum_{\epsilon \in S(\delta)}(\lambda, \delta-\epsilon) X_{\delta}
$$

By equating this to the evaluation of the right-hand side, we obtain

$$
\sum_{\epsilon \in S(\delta)}(\lambda, \delta-\epsilon)=(1 / 2) q(\mathfrak{g}, \mathcal{C})(\lambda, \delta) .
$$

Lemma 3.3 gives the value of the sum on the left and we obtain

$$
|S(\delta)|-c(\mathfrak{g}, \mathcal{C})+3=(1 / 2) q(\mathfrak{g}, \mathcal{C}) .
$$

Since $|S(\delta)|=(1 / 2)\left(\operatorname{dim}\left(V^{+}\right)-2\right)$, this gives the required formula. The last claim follows on comparing this formula with that given in Proposition 3.1.

Lemma 3.4. If $\alpha \in S(\delta)$ then

$$
\frac{N_{\delta^{\prime},-\alpha^{\prime}} N_{\delta,-\alpha}}{\|\alpha-\delta\|^{2}}=-(1 / 2) N_{\alpha, \alpha^{\prime}}
$$

Proof. The roots $\delta^{\prime},-\alpha^{\prime}, \delta$, and $-\alpha$ sum to zero and no two are opposite. By part (iv) of Theorem 4.1.2 of [5], we have the relation

$$
\frac{N_{\delta^{\prime},-\alpha^{\prime}} N_{\delta,-\alpha}}{\|\alpha-\delta\|^{2}}+\frac{N_{-\alpha^{\prime}, \delta} N_{\delta^{\prime},-\alpha}}{\left\|\alpha^{\prime}-\delta\right\|^{2}}+\frac{N_{\delta, \delta^{\prime}} N_{-\alpha^{\prime},-\alpha}}{\|\gamma\|^{2}}=0 .
$$

Since $\alpha-\delta^{\prime}$ is not a root, the middle term is zero. We have assumed that $N_{\delta, \delta^{\prime}}=1$ and so we obtain

$$
\frac{N_{\delta^{\prime},-\alpha^{\prime}} N_{\delta,-\alpha}}{\|\alpha-\delta\|^{2}}=-(1 / 2) N_{-\alpha^{\prime},-\alpha} .
$$

By using the properties of a Chevalley basis, we have

$$
N_{\alpha, \alpha^{\prime}}=-N_{-\alpha,-\alpha^{\prime}}=N_{-\alpha^{\prime},-\alpha}
$$

and the claim follows.

Lemma 3.5. For scalars $x$ and $y$ we have 
(1) $\tau_{1}\left(x X_{\delta}+y X_{\delta^{\prime}}\right)=y X_{-\delta}-x X_{-\delta^{\prime}}$,

(2) $\tau_{2}\left(x X_{\delta}+y X_{\delta^{\prime}}\right)=(x y / 2)\left(H_{\delta}-H_{\delta^{\prime}}\right)$,

(3) $\tau_{3}\left(x X_{\delta}+y X_{\delta^{\prime}}\right)=-\left(x^{2} y / 2\right) X_{\delta}+\left(x y^{2} / 2\right) X_{\delta^{\prime}}$,

(4) $\tau_{4}\left(x X_{\delta}+y X_{\delta^{\prime}}\right)=\left(x^{2} y^{2} / 4\right) X_{\gamma}$

Proof. In light of Lemma 3.1, it is sufficient to carry out the calculations in $\mathfrak{s t}(3)$. This is easily done.

Continuing with the above notation, we define

$$
X_{0}=\sqrt{2}\left(X_{\delta}+X_{\delta^{\prime}}\right) \in V^{+} .
$$

This point has been chosen so that $\Delta\left(X_{0}\right)=1$.

Proposition 3.3. The point $X_{0}$ is a generic point of the prehomogeneous vector space $\left(L, \mathrm{Ad}, V^{+}\right)$.

Proof. This follows, by the easy direction in Rubenthaler's Criterion [17, 18], from the observation that

$$
\left(2 H_{\gamma}, \sqrt{2}\left(X_{\delta}+X_{\delta^{\prime}}\right), \sqrt{2}\left(X_{-\delta}+X_{-\delta^{\prime}}\right)\right)
$$

is an $\mathfrak{s l}(2)$-triple. We remark that this implication appears as Problem 7 in Chapter X of [15], for which the proof of Theorem 10.10 in [15] may serve as a hint.

Lemma 3.6. For $\epsilon, \nu \in R\left(V^{+}\right)$we have

$$
\partial_{\epsilon} \tau_{2}(X)=(1 / 2)\left\langle X_{\epsilon}, X\right\rangle H_{\gamma}+N_{\epsilon,-\gamma}\left[X, X_{-\epsilon^{\prime}}\right]
$$

and

$$
\partial_{\epsilon \nu} \tau_{2}(X)=(1 / 2) \kappa_{\epsilon, \nu^{\prime}} N_{\epsilon, \nu} H_{\gamma}+N_{\epsilon,-\gamma}\left[X_{\nu}, X_{-\epsilon^{\prime}}\right] .
$$

Proof. The definition of $\tau_{2}$ implies that $\partial_{\epsilon} \tau_{2}(X)=(1 / 2)\left[X_{\epsilon}, \tau_{1}(X)\right]+$ $(1 / 2)\left[X, \tau_{1}\left(X_{\epsilon}\right)\right]$. However,

$$
\begin{aligned}
\left\langle X_{\epsilon}, X\right\rangle H_{\gamma} & =\left[X_{-\gamma},\left\langle X, X_{\epsilon}\right\rangle X_{\gamma}\right] \\
& =\left[X_{-\gamma},\left[X, X_{\epsilon}\right]\right] \\
& =\left[\left[X_{-\gamma}, X\right], X_{\epsilon}\right]+\left[X,\left[X_{-\gamma}, X_{\epsilon}\right]\right]
\end{aligned}
$$




$$
=\left[X_{\epsilon}, \tau_{1}(X)\right]-\left[X, \tau_{1}\left(X_{\epsilon}\right)\right]
$$

and so $\partial_{\epsilon} \tau_{2}(X)=(1 / 2)\left\langle X_{\epsilon}, X\right\rangle H_{\gamma}+\left[X, \tau_{1}\left(X_{\epsilon}\right)\right]$. The first formula follows on noting that $\tau_{1}\left(X_{\epsilon}\right)=N_{\epsilon,-\gamma} X_{-\epsilon^{\prime}}$. The second formula is an immediate consequence of the first.

Lemma 3.7. We have $\Delta_{\delta}\left(X_{0}\right)=\Delta_{\delta^{\prime}}\left(X_{0}\right)=\sqrt{2}$ and $\Delta_{\alpha}\left(X_{0}\right)=0$ for $\alpha \in R\left(V^{+}\right)-\left\{\delta, \delta^{\prime}\right\}$.

Proof. The first two values follow directly from Lemma 3.5. From the second formula of Lemma 2.6 we obtain

$$
\Delta_{\alpha}(X)=(1 / 3) \mathbf{B}_{\mathfrak{g}}\left(\left(\partial_{\alpha} \tau_{2}\right)(X), \tau_{2}(X)\right) .
$$

If $\alpha \notin\left\{\delta, \delta^{\prime}\right\}$ then it follows from Lemma 3.6 that $\left(\partial_{\alpha} \tau_{2}\right)\left(X_{0}\right)$ lies in either $\mathfrak{g}_{\delta-\alpha^{\prime}}$ or $\mathfrak{g}_{\delta^{\prime}-\alpha^{\prime}}$ and is therefore orthogonal to $\tau_{2}\left(X_{0}\right)=H_{\delta}-H_{\delta^{\prime}}$. This observation gives the remaining values.

The proof of the next result will follow a pattern that we shall use again several times. Thus it may be worth mentioning the simple general principle behind it. It is this: If $(L, \rho, W)$ is a representation of $L$ and $F: V^{+} \rightarrow W$ is an $L$-equivariant polynomial map such that $F\left(X_{0}\right)=0$ then $F$ vanishes identically. The proof is immediate from the fact that $\left(L, \mathrm{Ad}, V^{+}\right)$is prehomogeneous and $X_{0} \in V^{+}$is generic.

Proposition 3.4. For all $X \in V^{+}$we have

$$
\tau_{3}(X)=\sum_{\alpha \in R\left(V^{+}\right)} N_{\alpha, \alpha^{\prime}}^{-1}\left(\partial_{\alpha} \Delta\right)(X) X_{\alpha^{\prime}} .
$$

Proof. Lemma 3.5 and Lemma 3.7 imply that the two sides of the proposed identity agree at $X_{0}$. It therefore suffices to verify that they both transform in the same way under $L$. Lemma 2.3 implies that the left-hand side transforms by $\chi(l) \operatorname{Ad}(l)$ when $X$ is replaced by $\operatorname{Ad}(l) X$. Lemmas 2.9 and 2.7 imply that the right-hand side transforms in the same way under this substitution.

Lemma 3.8. We have

$$
\begin{aligned}
& \Delta_{\delta \delta}\left(X_{0}\right)=\Delta_{\delta^{\prime} \delta^{\prime}}\left(X_{0}\right)=1 \\
& \Delta_{\delta \delta^{\prime}}\left(X_{0}\right)=\Delta_{\delta^{\prime} \delta}\left(X_{0}\right)=2 .
\end{aligned}
$$

If $\alpha \in S(\delta)$ then $\Delta_{\alpha \alpha^{\prime}}\left(X_{0}\right)=N_{\alpha, \alpha^{\prime}}$. If $\alpha \in S\left(\delta^{\prime}\right)$ then $\Delta_{\alpha \alpha^{\prime}}\left(X_{0}\right)=-N_{\alpha, \alpha^{\prime}}$. All other second derivatives of $\Delta$ vanish at $X_{0}$. 
Proof. The values of $\Delta_{\delta \delta}\left(X_{0}\right), \ldots, \Delta_{\delta^{\prime} \delta^{\prime}}\left(X_{0}\right)$ follow from Lemma 3.5. From the second formula of Lemma 2.6 we obtain

$$
\begin{aligned}
& \Delta_{\alpha \beta}(X)= \\
& (1 / 3) \mathbf{B}_{\mathfrak{g}}\left(\left(\partial_{\alpha} \tau_{2}\right)(X),\left(\partial_{\beta} \tau_{2}\right)(X)\right)+(1 / 3) \mathbf{B}_{\mathfrak{g}}\left(\left(\partial_{\alpha \beta} \tau_{2}\right)(X), \tau_{2}(X)\right) .
\end{aligned}
$$

Let $\alpha, \beta \in R\left(V^{+}\right)-\left\{\delta, \delta^{\prime}\right\}$. We have $\tau_{2}\left(X_{0}\right)=H_{\delta}-H_{\delta^{\prime}}$ and, by Lemma 3.6,

$$
\begin{aligned}
& \left(\partial_{\alpha} \tau_{2}\right)\left(X_{0}\right)=\sqrt{2} N_{\alpha,-\gamma}\left[X_{\delta}+X_{\delta^{\prime}}, X_{-\alpha^{\prime}}\right], \\
& \left(\partial_{\beta} \tau_{2}\right)\left(X_{0}\right)=\sqrt{2} N_{\beta,-\gamma}\left[X_{\delta}+X_{\delta^{\prime}}, X_{-\beta^{\prime}}\right], \\
& \left(\partial_{\alpha \beta} \tau_{2}\right)\left(X_{0}\right)=(1 / 2) \kappa_{\alpha, \beta^{\prime}} N_{\alpha, \beta} H_{\gamma}+N_{\alpha,-\gamma}\left[X_{\beta}, X_{-\alpha^{\prime}}\right] .
\end{aligned}
$$

It is easy to see that if $\alpha \neq \beta^{\prime}$ then $\left(\partial_{\alpha} \tau_{2}\right)\left(X_{0}\right)$ and $\left(\partial_{\beta} \tau_{2}\right)\left(X_{0}\right)$ are orthogonal, and $\left(\partial_{\alpha \beta} \tau_{2}\right)\left(X_{0}\right)$ is orthogonal to $\tau_{2}\left(X_{0}\right)$. Thus $\Delta_{\alpha \beta}\left(X_{0}\right)=0$ in this case. Now assume that $\beta=\alpha^{\prime}$ and $\alpha \in S(\delta)$. Then

$$
\begin{aligned}
& \left(\partial_{\alpha} \tau_{2}\right)\left(X_{0}\right)=\sqrt{2} N_{\alpha,-\gamma} N_{\delta^{\prime},-\alpha^{\prime}} X_{\alpha-\delta}, \\
& \left(\partial_{\alpha^{\prime}} \tau_{2}\right)\left(X_{0}\right)=\sqrt{2} N_{\alpha^{\prime},-\gamma} N_{\delta,-\alpha} X_{\delta-\alpha}, \\
& \left(\partial_{\alpha \alpha^{\prime}} \tau_{2}\right)\left(X_{0}\right)=(1 / 2) N_{\alpha, \alpha^{\prime}} H_{\gamma}+N_{\alpha,-\gamma} H_{\alpha^{\prime}} .
\end{aligned}
$$

It follows that

$$
\begin{aligned}
\mathbf{B}_{\mathfrak{g}}\left(\left(\partial_{\alpha} \tau_{2}\right)\left(X_{0}\right),\left(\partial_{\alpha^{\prime}} \tau_{2}\right)\left(X_{0}\right)\right) & =4 N_{\alpha,-\gamma} N_{\alpha^{\prime},-\gamma} \frac{N_{\delta^{\prime},-\alpha^{\prime}} N_{\delta,-\alpha}}{\|\alpha-\delta\|^{2}} \\
& =-2 N_{\alpha,-\gamma} N_{\alpha^{\prime},-\gamma} N_{\alpha, \alpha^{\prime}} \\
& =(1 / 2)\|\alpha\|^{4} N_{\alpha, \alpha^{\prime}}^{3},
\end{aligned}
$$

where we have used Lemma 3.4 to get from the first line to the second, and $N_{\alpha,-\gamma}=-(1 / 2)\|\alpha\|^{2} N_{\alpha, \alpha^{\prime}}$ and $N_{\alpha^{\prime},-\gamma}=(1 / 2)\|\alpha\|^{2} N_{\alpha, \alpha^{\prime}}$ to get from the second line to the last. Now $\mathbf{B}_{\mathfrak{g}}\left(H_{\gamma}, \tau_{2}\left(X_{0}\right)\right)=0$ and so

$$
\begin{aligned}
\mathbf{B}_{\mathfrak{g}}\left(\left(\partial_{\alpha \alpha^{\prime}} \tau_{2}\right)\left(X_{0}\right), \tau_{2}\left(X_{0}\right)\right) & =N_{\alpha,-\gamma} \mathbf{B}_{\mathfrak{g}}\left(H_{\alpha^{\prime}}, H_{\delta}-H_{\delta^{\prime}}\right) \\
& =-N_{\alpha,-\gamma} \frac{4\left(\alpha^{\prime}, \delta^{\prime}\right)}{\|\alpha\|^{2}\|\delta\|^{2}} \\
& =-\left(2 /\|\alpha\|^{2}\right) N_{\alpha,-\gamma} \\
& =N_{\alpha, \alpha^{\prime}} .
\end{aligned}
$$

Thus

$$
\begin{aligned}
\Delta_{\alpha \alpha^{\prime}}\left(X_{0}\right) & =(1 / 6)\left(2 N_{\alpha, \alpha^{\prime}}+\|\alpha\|^{4} N_{\alpha, \alpha^{\prime}}^{3}\right) \\
& =(1 / 6) N_{\alpha, \alpha^{\prime}}\left(2+\|\alpha\|^{4} N_{\alpha, \alpha^{\prime}}^{2}\right) .
\end{aligned}
$$


By Lemma 2.2, $\|\alpha\|^{4} N_{\alpha, \alpha^{\prime}}^{2}=4$, and it follows that $\Delta_{\alpha \alpha^{\prime}}\left(X_{0}\right)=N_{\alpha, \alpha^{\prime}}$. This completes the evaluation when $\alpha \in S(\delta)$. Finally, if $\alpha \in S\left(\delta^{\prime}\right)$ then $\alpha^{\prime} \in S(\delta)$ and the required evaluation follows from the previous one and the equality of mixed partials.

Proposition 3.5. For all $X \in V^{+}$, we have

$$
\Delta_{\alpha \beta}(X)=(1 / 2)\left\langle X_{\alpha}, X\right\rangle\left\langle X_{\beta}, X\right\rangle-N_{\beta, \beta^{\prime}} M_{\alpha \beta^{\prime}}\left(\tau_{2}(X)\right) .
$$

Proof. The proof is in two steps. First, we verify that the two sides agree at $X_{0}$. Secondly, we verify that they transform in the same way under $L$. By Lemma 3.5, $\tau_{2}\left(X_{0}\right)=H_{\delta}-H_{\delta^{\prime}}$, and using this we obtain the expression

$$
\left(\kappa_{\alpha \delta}-\kappa_{\alpha \delta^{\prime}}\right)\left(\kappa_{\beta \delta}-\kappa_{\beta \delta^{\prime}}\right)-N_{\beta, \beta^{\prime}}\left(\kappa_{\alpha \beta^{\prime}}(\alpha, \delta)-\kappa_{\alpha \beta^{\prime}}\left(\alpha, \delta^{\prime}\right)\right)
$$

for the value of the right-hand side at $X_{0}$. It is routine to check that this agrees with the values of $\Delta_{\alpha \beta}\left(X_{0}\right)$ given in Lemma 3.8. This completes the first step.

The transformation law for $\Delta_{\alpha \beta}$ under the substitution $X \mapsto \operatorname{Ad}(l) X$ is given in Lemma 2.9, and it is easy to verify that the functions given by $X \mapsto\left\langle X_{\alpha}, X\right\rangle\left\langle X_{\beta}, X\right\rangle$ share the same transformation law. We finish the proof by determining the transformation law for the second term on the right-hand side, since this is more difficult. For $X \in V^{+}$and $l \in L$ we have

$$
\begin{aligned}
& N_{\beta, \beta^{\prime}} M_{\alpha \beta^{\prime}}\left(\tau_{2}(\operatorname{Ad}(l) X)\right) \\
& =N_{\beta, \beta^{\prime}} M_{\alpha \beta^{\prime}}\left(\chi(l) \operatorname{Ad}(l) \tau_{2}(X)\right) \\
& =\chi(l) N_{\beta, \beta^{\prime}}\left[m\left(l^{-1}\right) M\left(\tau_{2}(X)\right) m(l)\right]_{\alpha \beta^{\prime}} \\
& =\chi(l) \sum_{\mu, \nu} m_{\alpha \mu}\left(l^{-1}\right) M_{\mu \nu^{\prime}}\left(\tau_{2}(X)\right)\left(N_{\beta, \beta^{\prime}} m_{\nu^{\prime} \beta^{\prime}}(l)\right) \\
& =-\chi(l)^{2} \sum_{\mu, \nu} m_{\alpha \mu}\left(l^{-1}\right) M_{\mu \nu^{\prime}}\left(\tau_{2}(X)\right) N_{\nu^{\prime}, \nu} m_{\beta \nu}\left(l^{-1}\right) \\
& =\chi(l)^{2} \sum_{\mu, \nu} m_{\alpha \mu}\left(l^{-1}\right) m_{\beta \nu}\left(l^{-1}\right)\left(N_{\nu, \nu^{\prime}} M_{\mu \nu^{\prime}}\left(\tau_{2}(X)\right)\right) .
\end{aligned}
$$

During this computation we have made use of the identity $M(\operatorname{Ad}(l) X)=$ $m\left(l^{-1}\right) M(X) m(l)$ to get from the second line to the third, and Lemma 2.7 to get from the fourth line to the fifth. This is precisely the same transformation law that is enjoyed by the other terms in the proposed identity, and so the proof is complete.

For later use, we note that the formula given in Proposition 3.5 remains true when $\mathfrak{g}$ has type $\mathrm{C}_{r}$. In this case, $\Delta$ is identically zero and the formula is 
equivalent to

$$
\left\langle X_{\alpha}, X\right\rangle\left\langle X_{\beta}, X\right\rangle=2 N_{\beta, \beta^{\prime}} M_{\alpha \beta^{\prime}}\left(\tau_{2}(X)\right)
$$

for all $X \in V^{+}$. This may be verified by direct computation in this case.

\section{$\S 4$. The Fundamental Differential Operators}

In this section we shall embed $\mathfrak{g}$ into the algebra of differential operators on $\mathfrak{n}$ via the action derived from a suitable family of induced representations. In addition, we shall construct other differential operators on $\mathfrak{n}$ and study their interaction with the image of $\mathfrak{g}$.

Let $\mathbb{D}[\mathfrak{n}]$ be the Weyl algebra of $\mathfrak{n}$; that is, the algebra of partial differential operators on $\mathfrak{n}$ with polynomial coefficients. This algebra may be regarded as the quotient of the tensor algebra $T^{\bullet}\left(\mathfrak{n} \oplus \mathfrak{n}^{*}\right)$ by the ideal generated by the expressions $X_{1} \otimes X_{2}-X_{2} \otimes X_{1}$ for $X_{1}, X_{2} \in \mathfrak{n}, \lambda_{1} \otimes \lambda_{2}-\lambda_{2} \otimes \lambda_{1}$ for $\lambda_{1}, \lambda_{2} \in \mathfrak{n}^{*}$, and $X \otimes \lambda-\lambda \otimes X-\lambda(X) 1$ for $X \in \mathfrak{n}$ and $\lambda \in \mathfrak{n}^{*}$. The class of $X \in \mathfrak{n}$ in $\mathbb{D}[\mathfrak{n}]$ may be identified with the directional derivative $\partial_{X}$. As above, if $\alpha \in R\left(V^{+}\right)$ or $\alpha=\gamma$ then we use $\partial_{\alpha}$ as an abbreviation of $\partial_{X_{\alpha}}$, and let $\xi_{\alpha} \in \mathfrak{n}^{*}$ be the coordinate dual to $X_{\alpha}$. We have previously used $y$ and $\partial_{y}$ as synonyms for $\xi_{\gamma}$ and $\partial_{\gamma}$, respectively. If $\theta: \mathfrak{n} \rightarrow \mathfrak{n}$ is an invertible linear map then let $\tilde{\theta}: \mathfrak{n}^{*} \rightarrow \mathfrak{n}^{*}$ be the contragredient map; that is, the transpose of $\theta^{-1}$. It is easy to check that any invertible linear map $\theta: \mathfrak{n} \rightarrow \mathfrak{n}$ induces an automorphism of $\mathbb{D}[\mathfrak{n}]$ that agrees with $\theta$ on $\mathfrak{n}$ and with $\tilde{\theta}$ on $\mathfrak{n}^{*}$. In particular, we obtain an action of $L$ on $\mathbb{D}[\mathfrak{n}]$ induced by the adjoint action of $L$ on $\mathfrak{n}$. If $D \in \mathbb{D}[\mathfrak{n}]$ and $l \in L$ then we shall write $l \cdot D$ for the result of applying $l$ to $D$.

We shall have to consider several $\mathbb{D}[\mathfrak{n}]$-modules of functions on $\mathfrak{n}$, notably $\mathbb{C}[\mathfrak{n}]$ and $C^{\infty}(\mathfrak{n})$. If $f$ lies in such a module then we adopt the convention, common in the algebraic literature, of writing $D \bullet f$ for the result of acting on $f$ by $D \in \mathbb{D}[\mathfrak{n}]$. This allows us to preserve $D f$ for the product of $D$ and $f$ in $\mathbb{D}[\mathfrak{n}]$ in the case that $f$ happens to be a polynomial on $\mathfrak{n}$. Any module of functions on $\mathfrak{n}$ has an action of $L$ given by $(l \cdot f)(X)=f\left(\operatorname{Ad}\left(l^{-1}\right) X\right)$. This action is compatible with the $L$ action on $\mathbb{D}[\mathfrak{n}]$ in the sense that $l \cdot(D \bullet f)=(l \cdot D) \bullet(l \cdot f)$ for all $l \in L$ and $D \in \mathbb{D}[\mathfrak{n}]$. The action of $\mathbb{D}[\mathfrak{n}]$ on $\mathbb{C}[\mathfrak{n}]$ and, a fortiori, on $C^{\infty}(\mathfrak{n})$ is faithful. Thus we may regard $\mathbb{D}[\mathfrak{n}]$ as a subalgebra of the endomorphism algebra of either of these modules. However, some care is required with the natural $L$ actions under this identification, since they do not agree. One would normally define an $L$ action on $\operatorname{End}\left(C^{\infty}(\mathfrak{n})\right)$ by $(l * T)(f)=T\left(l^{-1} \cdot f\right)$ for $T \in \operatorname{End}\left(C^{\infty}(\mathfrak{n})\right)$. This action does not leave the image of $\mathbb{D}[\mathfrak{n}]$ in $\operatorname{End}\left(C^{\infty}(\mathfrak{n})\right)$ stable. In fact, the two actions are related by

$$
((l * D)(f))(X)=((l \cdot D) \bullet f)(\operatorname{Ad}(l) X) .
$$


Suppose that $f \in C^{\infty}(\mathfrak{n})$. Let $N$ be the connected subgroup of $G$ corresponding to $\mathfrak{n}$. Since exp $: \mathfrak{n} \rightarrow N$ is a diffeomorphism, there is a function $F \in C^{\infty}(N)$ that satisfies $f(X)=F(\exp (X))$ for all $X \in \mathfrak{n}$. This allows us to identify the spaces $C^{\infty}(N)$ and $C^{\infty}(\mathfrak{n})$. We shall repeatedly use the fact that the group structure on $N$ is determined by the identity

$$
\exp \left(X_{1}\right) \exp \left(X_{2}\right)=\exp \left(X_{1}+X_{2}+(1 / 2)\left[X_{1}, X_{2}\right]\right) .
$$

The group $N$ acts on itself on both the left and the right, and this gives rise to two derived actions of $\mathfrak{n}$ on $C^{\infty}(N)$, and hence on $C^{\infty}(\mathfrak{n})$. These are given, respectively, by

$$
\begin{aligned}
& (\mathbf{l}(Y) F)(x)=\mathrm{d}_{t=0}(F(\exp (-t Y) x)) \\
& (\mathbf{r}(Y) F)(x)=\mathrm{d}_{t=0}(F(x \exp (t Y))),
\end{aligned}
$$

where we use the symbol $\mathrm{d}_{t=0}$ as a shorthand for the operation of differentiating with respect to $t$ and then evaluating at $t=0$. The operators $\mathbf{l}(Y)$ and $\mathbf{r}(Y)$ lie in the image of $\mathbb{D}[\mathfrak{n}]$ in $\operatorname{End}\left(C^{\infty}(\mathfrak{n})\right)$ and we shall regard them henceforth as elements of $\mathbb{D}[\mathfrak{n}]$. Similar remarks will apply to the other operators that we construct below.

Lemma 4.1. For $Y, Y_{1}, Y_{2} \in \mathfrak{n}$ and $l \in L$ we have

(1) $\mathbf{l}(Y)=-\partial_{Y}-(1 / 2)\langle Y, \cdot\rangle \partial_{\gamma}$,

(2) $\mathbf{r}(Y)=\partial_{Y}-(1 / 2)\langle Y, \cdot\rangle \partial_{\gamma}$,

(3) $\left[\mathbf{l}\left(Y_{1}\right), \mathbf{r}\left(Y_{2}\right)\right]=0$,

(4) $\left[\mathbf{l}\left(Y_{1}\right), \mathbf{l}\left(Y_{2}\right)\right]=\mathbf{l}\left(\left[Y_{1}, Y_{2}\right]\right)$,

(5) $\left[\mathbf{r}\left(Y_{1}\right), \mathbf{r}\left(Y_{2}\right)\right]=\mathbf{r}\left(\left[Y_{1}, Y_{2}\right]\right)$,

(6) $l \cdot \mathbf{l}(Y)=\mathbf{l}(\operatorname{Ad}(l) Y)$,

(7) $l \cdot \mathbf{r}(Y)=\mathbf{r}(\operatorname{Ad}(l) Y)$.

Proof. Parts (1), (2), (4) and (5) are routine computations, and part (3) follows from the fact that the underlying geometric actions from which $\mathbf{l}\left(Y_{1}\right)$ and $\mathbf{r}\left(Y_{2}\right)$ derive themselves commute. For parts (6) and (7), note first that by definition $l \cdot \partial_{Y}=\partial_{\operatorname{Ad}(l) Y}$ and $l \cdot \partial_{\gamma}=\chi(l) \partial_{\gamma}$. If we define $p \in \mathbb{C}[\mathfrak{n}]$ by $p(X)=\langle Y, X\rangle$ then

$$
(l \cdot p)(X)=\left\langle Y, \operatorname{Ad}\left(l^{-1}\right) X\right\rangle=\chi(l)^{-1}\langle\operatorname{Ad}(l) Y, X\rangle .
$$


These identities combine to give the last two parts.

We emphasize again that the $L$ action referred to in (6) and (7) of the lemma is the $L$ action on $\mathbb{D}[\mathfrak{n}]$, not the $L$ action on $\operatorname{End}\left(C^{\infty}(\mathfrak{n})\right)$.

Next we wish to extend $\mathbf{l}$ to a family of homomorphisms from $\mathfrak{g}$ into the Lie algebra underlying $\mathbb{D}[\mathfrak{n}]$, parameterized by a complex number $s$. Let $\bar{Q}$ be the parabolic subgroup of $G$ associated to the parabolic subalgebra $\overline{\mathfrak{q}}=\mathfrak{l} \oplus \overline{\mathfrak{n}}$ of $\mathfrak{g}$ and $\bar{N}$ be the connected subgroup of $G$ corresponding to the subalgebra $\overline{\mathfrak{n}}$ of $\mathfrak{g}$. We have a Levi decomposition $\bar{Q}=L \bar{N}$ and we may extend $\chi$ to be a character of $\bar{Q}$ by requiring that it be trivial on $\bar{N}$. Naively, the family of homomorphisms that we require is the one derived from the family of (unnormalized) induced representations $C^{\infty}-\operatorname{Ind}\left(G, \bar{Q} ; \chi^{-s}\right)$. This cannot be taken literally, as there is no way to define the family $\chi^{-s}$ of characters on the complex group $\bar{Q}$. More precisely, we consider the real structure on $\mathfrak{g}$ that corresponds to our chosen Chevalley basis and call the set of real points $\mathfrak{g}_{0}$. This is a split real Lie algebra with the same Dynkin diagram as $\mathfrak{g}$, and all our preceding algebraic constructions carry over without change to $\mathfrak{g}_{0}$, yielding objects defined over $\mathbb{R}$ in all cases. Let us use the subscript 0 to denote real points in general. The character $\chi$ is real-valued on $L_{0}$ and we may consider the family of homomorphisms from $\mathfrak{g}_{0}$ to $\mathbb{D}\left[\mathfrak{n}_{0}\right]$ that derives from the family of induced representations $C^{\infty}-\operatorname{Ind}\left(G_{0}, \bar{Q}_{0} ;|\chi|^{-s}\right)$. Complexifying this family leads to the family of homomorphisms from $\mathfrak{g}$ to $\mathbb{D}[\mathfrak{n}]$ that we require.

Let $s \in \mathbb{C}$ and $F \in C^{\infty}\left(N_{0}\right)$. The Bruhat decomposition associated to the parabolic subgroup $\bar{Q}_{0}$ implies that $N_{0} \bar{Q}_{0}$ is a dense open subset of $G_{0}$. Note that this subset is stable under left multiplication by $L_{0}$. For each $x \in N_{0}$ there is an open set $U_{x} \subset G_{0}$ containing $L_{0}$ such that $g \in U_{x}$ implies that $g^{-1} x \in N_{0} \bar{Q}_{0}$. If $g \in U_{x}$ then we may write

$$
g^{-1} x=\nu(g, x) \bar{q}(g, x)
$$

with $\nu(g, x) \in N_{0}$ and $\bar{q}(g, x) \in \bar{Q}_{0}$. In terms of this decomposition we define

$$
\left(\pi_{s}(g) F\right)(x)=|\chi(\bar{q}(g, x))|^{s} F(\nu(g, x)),
$$

thereby obtaining the germ of a representation of $G_{0}$ on $C^{\infty}\left(N_{0}\right)$. If we added appropriate growth conditions then this would simply be the so-called noncompact model of the smooth induced representation $C^{\infty}-\operatorname{Ind}\left(G_{0}, \bar{Q}_{0} ;|\chi|^{-s}\right)$. However, for our current purposes, the growth conditions are irrelevant. Let $\Pi_{s}: \mathfrak{g}_{0} \rightarrow \operatorname{End}\left(C^{\infty}\left(N_{0}\right)\right)$ be the derived representation of $\pi_{s}$, which we may also regard as mapping into $\operatorname{End}\left(C^{\infty}\left(\mathfrak{n}_{0}\right)\right)$. We shall see that the image of 
$\Pi_{s}$ lies in $\mathbb{D}\left[\mathfrak{n}_{0}\right]$. Once we know this, we may complexify to obtain the map $\Pi_{s}: \mathfrak{g} \rightarrow \mathbb{D}[\mathfrak{n}]$ that is our current goal.

It follows directly from the definitions that $\Pi_{s}(Y)=\mathbf{l}(Y)$ for $Y \in \mathfrak{n}_{0}$, and this identity extends to $Y \in \mathfrak{n}$ on complexification. Our next task is to determine $\Pi_{s}(Z)$ for $Z \in \mathfrak{l}$.

Lemma 4.2. If $Z \in \mathfrak{l}$ then $\Pi_{s}(Z)=-\partial_{[Z, \cdot]}-s \lambda_{\chi}(Z)$.

Proof. If $l \in L_{0}$ and $x \in N_{0}$ then $l^{-1} x=\left(l^{-1} x l\right) l^{-1}$ and so $\nu(l, x)=$ $l^{-1} x l$ and $\bar{q}(l, x)=l^{-1}$. Thus for $F \in C^{\infty}\left(N_{0}\right)$ we have $\left(\pi_{s}(l) F\right)(x)=$ $|\chi(l)|^{-s} F\left(l^{-1} x l\right)$. Let $Z \in \mathfrak{l}_{0}$ and $X \in \mathfrak{n}_{0}$ and put $l=\exp (t Z)$ and $x=\exp (X)$ in the previous equation. If $f \in C^{\infty}\left(\mathfrak{n}_{0}\right)$ is the function corresponding to $F$ then the result may be written as

$$
\left(\pi_{s}(\exp (t Z)) F\right)(\exp (X))=\chi(\exp (t Z))^{-s} f(\operatorname{Ad}(\exp (-t Z))(X)) .
$$

Differentiating this expression with respect to $t$ and setting $t=0$, we obtain

$$
\left(\Pi_{s}(Z) \bullet f\right)(X)=-s \lambda_{\chi}(Z) f(X)-\left(\partial_{[Z, X]} \bullet f\right)(X),
$$

which is the required expression when $Z \in \mathfrak{l}_{0}$. The general identity follows at once on complexification.

In order to understand $\Pi_{s}$ we still require a tractable expression for $\Pi_{s}(Y)$ when $Y \in \overline{\mathfrak{n}}$. Actually, by Lemma 2.1, it suffices to have such an expression for $\Pi_{s}\left(X_{-\gamma}\right)$. The next two results give these expressions. The first is a general, but somewhat unwieldy, formula for $\Pi_{s}(Y)$; the second is a much simpler formula for $\Pi_{s}\left(X_{-\gamma}\right)$. We have the direct decompositions

$$
\mathfrak{g}=\mathfrak{n} \oplus \overline{\mathfrak{q}}=\mathfrak{n} \oplus \mathfrak{l} \oplus \overline{\mathfrak{n}}
$$

and we let $\mathrm{pr}_{\mathfrak{n}}, \mathrm{pr}_{\overline{\mathfrak{q}}}$, and $\mathrm{pr}_{\mathfrak{l}}$ be the projection operators onto the indicated subalgebras. On occasion, we write $e^{X}$ in place of $\exp (X)$ to save space.

Proposition 4.1. Let $Y \in \mathfrak{g}, X \in \mathfrak{n}$ and $f \in C^{\infty}(\mathfrak{n})$. Then

$$
\begin{aligned}
\left(\Pi_{s}(Y) \bullet f\right) & (X)= \\
& -\left(\mathbf{r}\left(\operatorname{pr}_{\mathfrak{n}}\left(\operatorname{Ad}\left(e^{-X}\right)(Y)\right)\right) \cdot f\right)(X)-s \lambda_{\chi}\left(\operatorname{pr}_{\mathfrak{l}}\left(\operatorname{Ad}\left(e^{-X}\right)(Y)\right)\right) f(X) .
\end{aligned}
$$

Proof. As usual we first work in the real setting, observe that the resulting operator $\Pi_{s}(Y)$ lies in $\mathbb{D}\left[\mathfrak{n}_{0}\right]$ and is given by the correct expression, and finally 
obtain the expression in general on complexification. We have

$$
\begin{aligned}
& \exp (-t Y) \exp (X) \\
& =\exp (X) \exp (-X) \exp (-t Y) \exp (X) \\
& =\exp (X) \exp \left(-t \operatorname{Ad}\left(e^{-X}\right)(Y)\right) \\
& =\exp (X) \exp \left(-t \operatorname{pr}_{\mathfrak{n}}\left(\operatorname{Ad}\left(e^{-X}\right)(Y)\right)-t \operatorname{pr}_{\overline{\mathfrak{q}}}\left(\operatorname{Ad}\left(e^{-X}\right)(Y)\right)\right)
\end{aligned}
$$

It follows from the Bruhat decomposition that there is some $\epsilon>0$ and a map $\theta_{\mathfrak{n}}:(-\epsilon, \epsilon) \rightarrow N_{0}$ such that

$$
\nu(\exp (t Y), \exp (X))=\exp (X) \exp \left(-\operatorname{tpr}_{\mathfrak{n}}\left(\operatorname{Ad}\left(e^{-X}\right)(Y)\right)\right) \theta_{\mathfrak{n}}(t)
$$

for all $t \in(-\epsilon, \epsilon)$. Comparing this expression and the previous one, we conclude that $\theta_{\mathfrak{n}}(0)=e$ and $\theta_{\mathfrak{n}}^{\prime}(0)=0$. Similarly, after possibly decreasing $\epsilon$, there is a map $\theta_{\overline{\mathfrak{q}}}:(-\epsilon, \epsilon) \rightarrow \bar{Q}_{0}$ such that

$$
\bar{q}(\exp (t Y), \exp (X))=\exp \left(-t \operatorname{pr}_{\overline{\mathfrak{q}}}\left(\operatorname{Ad}\left(e^{-X}\right)(Y)\right)\right) \theta_{\overline{\mathfrak{q}}}(t)
$$

for all $t \in(-\epsilon, \epsilon)$, and we have $\theta_{\overline{\mathfrak{q}}}(0)=e$ and $\theta_{\overline{\mathfrak{q}}}^{\prime}(0)=0$.

Let $F \in C^{\infty}\left(N_{0}\right)$ be the function that corresponds to $f$. Then

$$
\begin{aligned}
& \left(\pi_{s}(\exp (t Y)) F\right)(\exp (X))= \\
& \chi(\bar{q}(\exp (t Y), \exp (X)))^{s} F(\nu(\exp (t Y), \exp (X)))
\end{aligned}
$$

and $\left(\Pi_{s}(Y) \bullet f\right)(X)$ is the result of applying $\mathrm{d}_{t=0}$ to this. Since $\chi$ has been extended to be trivial on $\bar{N}_{0}$,

$$
\mathrm{d}_{t=0} \chi(\bar{q}(\exp (t Y), \exp (X)))^{s}=-s \lambda_{\chi}\left(\operatorname{pr}_{\mathfrak{l}}\left(\operatorname{Ad}\left(e^{-X}\right)(Y)\right)\right) .
$$

The definition of $\mathbf{r}$ implies that

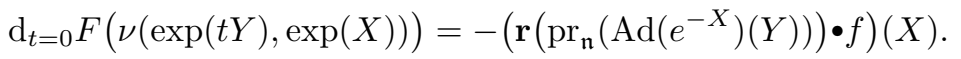

Combining these two evaluations gives the required expression for real $X$ and $Y$. Notice that this expression implies that $\Pi_{s}(Y)$ lies in $\mathbb{D}\left[\mathfrak{n}_{0}\right]$, which is the last ingredient required to complete the argument.

If $f_{1}, f_{2} \in C^{\infty}(\mathfrak{n})$ then we define

$$
\left\{f_{1}, f_{2}\right\}=\sum_{\alpha \in R\left(V^{+}\right)} N_{\alpha, \alpha^{\prime}}^{-1}\left(\partial_{\alpha} \bullet f_{1}\right)\left(\partial_{\alpha^{\prime}} \bullet f_{2}\right) .
$$


This bilinear form is essentially the Poisson bracket associated to the degenerate alternating form $\langle\cdot, \cdot\rangle$ on $\mathfrak{n}$. If $f \in \mathbb{C}[\mathfrak{n}]$ then

$$
\{f, \cdot\}=\sum_{\alpha \in R\left(V^{+}\right)} N_{\alpha, \alpha^{\prime}}^{-1}\left(\partial_{\alpha} \bullet f\right) \partial_{\alpha^{\prime}}
$$

is an element of $\mathbb{D}[\mathfrak{n}]$ having the property that $\{f, \cdot\} \bullet \phi=\{f, \phi\}$ for all $\phi \in$ $C^{\infty}(\mathfrak{n})$. Define $E \in \mathbb{D}[\mathfrak{n}]$ by

$$
E=2 \xi_{\gamma} \partial_{\gamma}+\sum_{\alpha \in R\left(V^{+}\right)} \xi_{\alpha} \partial_{\alpha} .
$$

This is the Euler operator associated to the grading of $\mathbb{C}[\mathfrak{n}]$ that assigns the $H_{\gamma^{-}}$ eigenvalue as the weight. Explicitly, this weight function satisfies $\operatorname{wt}\left(\xi_{\alpha}\right)=1$ for $\alpha \in R\left(V^{+}\right)$and $\operatorname{wt}\left(\xi_{\gamma}\right)=2$. If $f \in \mathbb{C}[\mathfrak{n}]$ is homogeneous with respect to this weight function then $[E, f]=E \bullet f=\operatorname{wt}(f) f$. We may extend wt to $\mathbb{C}\left[\mathfrak{n}^{*}\right]$ by defining $\operatorname{wt}\left(\partial_{\alpha}\right)=-1$ for $\alpha \in R\left(V^{+}\right)$and $\operatorname{wt}\left(\partial_{\gamma}\right)=-2$. If $D \in \mathbb{C}\left[\mathfrak{n}^{*}\right]$ is homogeneous for wt then we have $[E, D]=\operatorname{wt}(D) D$.

Proposition 4.2. In $\mathbb{D}[\mathfrak{n}]$ we have

$$
\Pi_{s}\left(X_{-\gamma}\right)=-P \partial_{\gamma}+\xi_{\gamma} E+\{\Delta, \cdot\}+2 s \xi_{\gamma},
$$

where $P=\xi_{\gamma}^{2}-\Delta$

Proof. Let $X \in V^{+}$. From (2.2) we have the identity

$$
\begin{aligned}
& \operatorname{Ad}\left(e^{-\left(X+\xi_{\gamma} X_{\gamma}\right)}\right)\left(X_{-\gamma}\right)= \\
& \quad X_{-\gamma}-\tau_{1}(X)+\left(\tau_{2}(X)-\xi_{\gamma} H_{\gamma}\right)-\left(\xi_{\gamma} X+\tau_{3}(X)\right)+\left(\Delta(X)-\xi_{\gamma}^{2}\right) X_{\gamma}
\end{aligned}
$$

from which it follows that

$$
\begin{aligned}
& \operatorname{pr}_{\mathfrak{l}}\left(\operatorname{Ad}\left(e^{-\left(X+\xi_{\gamma} X_{\gamma}\right)}\right)\left(X_{-\gamma}\right)\right)=\tau_{2}(X)-\xi_{\gamma} H_{\gamma}, \\
& \operatorname{pr}_{\mathfrak{n}}\left(\operatorname{Ad}\left(e^{-\left(X+\xi_{\gamma} X_{\gamma}\right)}\right)\left(X_{-\gamma}\right)\right)=-\xi_{\gamma} X-\tau_{3}(X)+\left(\Delta(X)-\xi_{\gamma}^{2}\right) X_{\gamma} .
\end{aligned}
$$

Now $\left[H_{\gamma}, X_{\gamma}\right]=2 X_{\gamma}$ and so $\lambda_{\chi}\left(H_{\gamma}\right)=2$. On the other hand, we have $\left[\tau_{2}(X), X_{\gamma}\right]=0$ by Lemma 2.4 and so $\lambda_{\chi}\left(\tau_{2}(X)\right)=0$. These evaluations imply that

$$
\lambda_{\chi}\left(\operatorname{pr}_{\mathfrak{l}}\left(\operatorname{Ad}\left(e^{-\left(X+\xi_{\gamma} X_{\gamma}\right)}\right)\left(X_{-\gamma}\right)\right)\right)=-2 \xi_{\gamma},
$$

which gives us the value of the second term in the expression from Proposition 4.1 with $Y=X_{-\gamma}$. The first term in that expression is now revealed to be a sum 
of three terms, namely $\xi_{\gamma} \mathbf{r}(X), \mathbf{r}\left(\tau_{3}(X)\right)$ and $-\left(\Delta(X)-\xi_{\gamma}^{2}\right) \mathbf{r}\left(X_{\gamma}\right)$. To evaluate these terms, we begin with the formula for $\mathbf{r}$ given in part (2) of Lemma 4.1. From this formula we obtain $\mathbf{r}\left(X_{\gamma}\right)=\partial_{\gamma}$ and, since $\langle X, X\rangle=0$,

$$
\mathbf{r}(X)=\partial_{X}=\sum_{\alpha \in R\left(V^{+}\right)} \xi_{\alpha} \partial_{\alpha}=E-2 \xi_{\gamma} \partial_{\gamma}
$$

Moreover, $\mathbf{r}\left(\tau_{3}(X)\right)=\partial_{\tau_{3}(X)}-(1 / 2)\left\langle\tau_{3}(X), X\right\rangle \partial_{\gamma}$. By definition,

$$
\begin{aligned}
\left\langle\tau_{3}(X), X\right\rangle X_{\gamma} & =\left[\tau_{3}(X), X\right] \\
& =-4 \tau_{4}(X) \\
& =-4 \Delta(X) X_{\gamma}
\end{aligned}
$$

so that $\left\langle\tau_{3}(X), X\right\rangle=-4 \Delta(X)$. By using the expression for $\tau_{3}(X)$ given in Proposition 3.4, we obtain

$$
\partial_{\tau_{3}(X)}=\sum_{\alpha \in R\left(V^{+}\right)} N_{\alpha, \alpha^{\prime}}^{-1}\left(\partial_{\alpha} \bullet \Delta\right) \partial_{\alpha^{\prime}}=\{\Delta, \cdot\} .
$$

These evaluations combine to give $\mathbf{r}\left(\tau_{3}(X)\right)=\{\Delta, \cdot\}+2 \Delta \partial_{\gamma}$. By adding all these terms and simplifying we obtain the expression in the statement.

For $\alpha \in R\left(V^{+}\right)$define $\nabla_{\alpha} \in \mathbb{D}[\mathfrak{n}]$ by

$$
\nabla_{\alpha}=\mathbf{r}\left(X_{\alpha}\right)=\partial_{\alpha}-(1 / 2) N_{\alpha, \alpha^{\prime}} \xi_{\alpha^{\prime}} \partial_{\gamma}
$$

For $\mu \in R$ define $D_{\mu}=\Pi_{s}\left(X_{\mu}\right)$. Although $D_{\mu}$ may depend on $s$, we suppress this dependence in the notation. If $f \in C^{\infty}(\mathfrak{n})$ and $\alpha, \beta \in R(\mathfrak{n})$ then let $f_{\alpha}=\partial_{\alpha} \bullet f, f_{\alpha \beta}=\left(\partial_{\beta} \partial_{\alpha}\right) \bullet f$, and similarly for higher partial derivatives.

Proposition 4.3. Let $\alpha \in R\left(V^{+}\right), \beta \in R(\mathfrak{n})$, and $Z \in \mathfrak{l}$. Then we have $\left[D_{\beta}, \nabla_{\alpha}\right]=0$ and

$$
\left[\Pi_{s}(Z), \nabla_{\alpha}\right]=\sum_{\mu \in R\left(V^{+}\right)} M_{\alpha \mu}(Z) \nabla_{\mu}
$$

Proof. The first claim follows at once from part (3) of Lemma 4.1. As an element of $\mathbb{D}[\mathfrak{n}]$, we have

$$
\partial_{[Z, \cdot]}=\lambda_{\chi}(Z) \xi_{\gamma} \partial_{\gamma}+\sum_{\mu, \nu \in R\left(V^{+}\right)} M_{\mu \nu}(Z) \xi_{\mu} \partial_{\nu}
$$


and so

$$
\begin{aligned}
& {\left[\Pi_{s}(Z), \nabla_{\alpha}\right]} \\
& \begin{aligned}
&=-\left[\partial_{[Z, \cdot]}, \partial_{\alpha}-(1 / 2) N_{\alpha, \alpha^{\prime}} \xi_{\alpha^{\prime}} \partial_{\gamma}\right] \\
&=\sum_{\nu \in R\left(V^{+}\right)} M_{\alpha \nu}(Z) \partial_{\nu}-(1 / 2) N_{\alpha, \alpha^{\prime}} \lambda_{\chi}(Z) \xi_{\alpha^{\prime}} \partial_{\gamma} \\
&+(1 / 2) N_{\alpha, \alpha^{\prime}} \sum_{\mu \in R\left(V^{+}\right)} M_{\mu \alpha^{\prime}}(Z) \xi_{\mu} \partial_{\gamma}
\end{aligned}
\end{aligned}
$$

by direct calculation. On the other hand,

$$
\begin{aligned}
& \sum_{\mu \in R\left(V^{+}\right)} M_{\alpha \mu}(Z) \nabla_{\mu} \\
= & \sum_{\mu \in R\left(V^{+}\right)} M_{\alpha \mu}(Z)\left(\partial_{\mu}-(1 / 2) N_{\mu, \mu^{\prime}} \xi_{\mu^{\prime}} \partial_{\gamma}\right) \\
= & \sum_{\mu \in R\left(V^{+}\right)} M_{\alpha \mu}(Z) \partial_{\mu} \\
& -(1 / 2) \sum_{\mu \in R\left(V^{+}\right)}\left(-N_{\alpha, \alpha^{\prime}} M_{\mu^{\prime} \alpha^{\prime}}(Z) \xi_{\mu^{\prime}}+\kappa_{\alpha \mu} N_{\alpha, \alpha^{\prime}} \lambda_{\chi}(Z) \xi_{\mu^{\prime}}\right) \partial_{\gamma} \\
= & \sum_{\mu \in R\left(V^{+}\right)} M_{\alpha \mu}(Z) \partial_{\mu}-(1 / 2) N_{\alpha, \alpha^{\prime}} \lambda_{\chi}(Z) \xi_{\alpha^{\prime}} \partial_{\gamma} \\
& \quad+(1 / 2) N_{\alpha, \alpha^{\prime}} \sum_{\mu \in R\left(V^{+}\right)} M_{\mu \alpha^{\prime}}(Z) \xi_{\mu} \partial_{\gamma},
\end{aligned}
$$

where we have used Lemma 2.7 to get from the second line to the third. This establishes the second claim.

Theorem 4.1. For $\alpha, \mu \in R\left(V^{+}\right)$let

$$
\Lambda_{\alpha \mu}=-\kappa_{\alpha \mu} \xi_{\gamma}+M_{\alpha \mu}\left(\tau_{2}\right) \text {, }
$$

where $M_{\alpha \mu}\left(\tau_{2}\right)$ denotes the polynomial $X \mapsto M_{\alpha \mu}\left(\tau_{2}(X)\right)$. Then

$$
\left[D_{-\gamma}, \nabla_{\alpha}\right]=\sum_{\mu \in R\left(V^{+}\right)} \Lambda_{\alpha \mu} \nabla_{\mu}+s N_{\alpha, \alpha^{\prime}} \xi_{\alpha^{\prime}}
$$

Proof. Let

$$
\tilde{\Lambda}_{\alpha \mu}=-\kappa_{\alpha \mu} \xi_{\gamma}+(1 / 2) N_{\alpha, \alpha^{\prime}} \xi_{\alpha^{\prime}} \xi_{\mu}+N_{\mu, \mu^{\prime}}^{-1} \Delta_{\alpha \mu^{\prime}} .
$$

We first establish the identity with $\tilde{\Lambda}$ in place of $\Lambda$ and then show that $\tilde{\Lambda}=\Lambda$. Let us compute the left-hand side explicitly using the expression for $D_{-\gamma}$ given 
in Proposition 4.2. Most of the computations involved in finding the individual commutators are routine, and for these we merely give the result. We have $\left[P \partial_{\gamma}, \partial_{\alpha}\right]=\Delta_{\alpha} \partial_{\gamma},\left[\xi_{\gamma} E, \partial_{\alpha}\right]=-\xi_{\gamma} \partial_{\alpha}$, and

$$
\begin{aligned}
{\left[\{\Delta, \cdot\}, \partial_{\alpha}\right] } & =-\left\{\Delta_{\alpha}, \cdot\right\} \\
& =-\sum_{\mu} N_{\mu, \mu^{\prime}}^{-1} \Delta_{\alpha \mu} \partial_{\mu^{\prime}} \\
& =\sum_{\mu} N_{\mu, \mu^{\prime}}^{-1} \Delta_{\alpha \mu^{\prime}} \partial_{\mu},
\end{aligned}
$$

where the sums are over $\mu \in R\left(V^{+}\right)$and we have replaced $\mu$ by $\mu^{\prime}$ and used the skew-symmetry of $N$ to obtain the last line. These evaluations combine to give

$$
\left[D_{-\gamma}, \partial_{\alpha}\right]=-\xi_{\gamma} \partial_{\alpha}+\sum_{\mu} N_{\mu, \mu^{\prime}}^{-1} \Delta_{\alpha \mu^{\prime}} \partial_{\mu}-\Delta_{\alpha} \partial_{\gamma}
$$

Similarly, we have $\left[P \partial_{\gamma}, \xi_{\alpha^{\prime}} \partial_{\gamma}\right]=-2 \xi_{\alpha^{\prime}} \xi_{\gamma} \partial_{\gamma},\left[\xi_{\gamma} E, \xi_{\alpha^{\prime}} \partial_{\gamma}\right]=-\xi_{\alpha^{\prime}} \xi_{\gamma} \partial_{\gamma}-\xi_{\alpha^{\prime}} E$, $\left[\xi_{\gamma}, \xi_{\alpha^{\prime}} \partial_{\gamma}\right]=-\xi_{\alpha^{\prime}}$, and $\left[\{\Delta, \cdot\}, \xi_{\alpha^{\prime}} \partial_{\gamma}\right]=\left\{\Delta, \xi_{\alpha^{\prime}}\right\} \partial_{\gamma}=N_{\alpha, \alpha^{\prime}}^{-1} \Delta_{\alpha} \partial_{\gamma}$. These evaluations yield

$$
\left[D_{-\gamma}, \xi_{\alpha^{\prime}} \partial_{\gamma}\right]=\xi_{\alpha^{\prime}} \xi_{\gamma} \partial_{\gamma}-\xi_{\alpha^{\prime}} E+N_{\alpha, \alpha^{\prime}}^{-1} \Delta_{\alpha} \partial_{\gamma}-2 s \xi_{\alpha^{\prime}}
$$

and so

$$
\begin{aligned}
{\left[D_{-\gamma}, \nabla_{\alpha}\right] } & =-\xi_{\gamma} \partial_{\alpha}+(1 / 2) N_{\alpha, \alpha^{\prime}} \xi_{\alpha^{\prime}} \sum_{\lambda} \xi_{\lambda} \partial_{\lambda}+\sum_{\mu} N_{\mu, \mu^{\prime}}^{-1} \Delta_{\alpha \mu^{\prime}} \partial_{\mu} \\
& -(3 / 2) \Delta_{\alpha} \partial_{\gamma}+(1 / 2) N_{\alpha, \alpha^{\prime}} \xi_{\alpha^{\prime}} \xi_{\gamma} \partial_{\gamma}+s N_{\alpha, \alpha^{\prime}} \xi_{\alpha^{\prime}} .
\end{aligned}
$$

Now $\tilde{\Lambda}_{\alpha \mu}$ is the coefficient of $\partial_{\mu}$ on the right-hand side of this equation, and the final term involving $s$ is as stated. In order to verify the identity with $\tilde{\Lambda}$ in place of $\Lambda$, it remains to show that the coefficient of $\partial_{\gamma}$ is correct. This amounts to the equation

$$
-\frac{1}{2} \sum_{\mu} N_{\mu, \mu^{\prime}} \tilde{\Lambda}_{\alpha \mu} \xi_{\mu^{\prime}}=-\frac{3}{2} \Delta_{\alpha}+\frac{1}{2} N_{\alpha, \alpha^{\prime}} \xi_{\alpha^{\prime}} \xi_{\gamma} .
$$

The term in $\tilde{\Lambda}_{\alpha \mu}$ involving the Kronecker symbol gives the second term on the right, and

$$
\begin{aligned}
& \sum_{\mu} N_{\mu, \mu^{\prime}}\left((1 / 2) N_{\alpha, \alpha^{\prime}} \xi_{\alpha^{\prime}} \xi_{\mu}+N_{\mu, \mu^{\prime}}^{-1} \Delta_{\alpha \mu^{\prime}}\right) \xi_{\mu^{\prime}} \\
& =(1 / 2) N_{\alpha, \alpha^{\prime}} \xi_{\alpha^{\prime}} \sum_{\mu} N_{\mu, \mu^{\prime}} \xi_{\mu} \xi_{\mu^{\prime}}+\sum_{\mu} \Delta_{\alpha \mu^{\prime}} \xi_{\mu^{\prime}} \\
& =3 \Delta_{\alpha},
\end{aligned}
$$


since the first term on the second line is zero by the skew-symmetry of $N_{\mu, \mu^{\prime}}$ and the second term is $3 \Delta_{\alpha}$ by Euler's identity. This completes the first step. It remains to show that $\tilde{\Lambda}=\Lambda$. Well, by Proposition 3.5 ,

$$
\begin{aligned}
\Delta_{\alpha \mu^{\prime}} & =(1 / 2)\left\langle X_{\alpha}, \cdot\right\rangle\left\langle X_{\mu^{\prime}}, \cdot\right\rangle-N_{\mu^{\prime}, \mu} M_{\alpha \mu}\left(\tau_{2}\right) \\
& =(1 / 2) N_{\alpha, \alpha^{\prime}} N_{\mu^{\prime}, \mu} \xi_{\alpha^{\prime}} \xi_{\mu}+N_{\mu, \mu^{\prime}} M_{\alpha \mu}\left(\tau_{2}\right),
\end{aligned}
$$

and substituting this into the definition of $\tilde{\Lambda}_{\alpha \mu}$ gives what is required.

\section{$\S 5 . \quad$ Conformally Invariant Systems I}

In this section, we shall construct maps

$$
\begin{aligned}
& \Omega_{1}: V^{+} \rightarrow \mathbb{D}[\mathfrak{n}], \\
& \Omega_{2}: \mathfrak{l} \rightarrow \mathbb{D}[\mathfrak{n}],
\end{aligned}
$$

corresponding to the covariants $\tau_{1}$ and $\tau_{2}$, respectively. Each of these maps will give one or more conformally invariant systems of differential equations for a specific value of the parameter $s$. Note that the domain of $\Omega_{j}$ is the dual space with respect to $\mathbf{B}_{\mathfrak{g}}$ of the codomain of $\tau_{j}$. By duality, each element of the domain is thus made to correspond to a polynomial on $V^{+}$. We then define $\Omega_{j}$ by a symbolic substitution process, chosen to respect the $L$ action. The variables $\xi_{\alpha}$ span a copy of $\left(V^{+}\right)^{*}$ in $\mathbb{D}[\mathfrak{n}]$, whereas, by part (7) of Lemma 4.1, the $\nabla_{\alpha}$ span a copy of $V^{+}$. The isomorphism $\varphi: V^{+} \rightarrow \chi \otimes\left(V^{+}\right)^{*}$ that derives from the existence of the bilinear form $\langle\cdot, \cdot\rangle$ satisfies $\varphi\left(X_{\alpha}\right)=N_{\alpha, \alpha^{\prime}} \xi_{\alpha^{\prime}}$, and so we replace $\xi_{\alpha}$ by $-N_{\alpha, \alpha^{\prime}}^{-1}, \nabla_{\alpha^{\prime}}$. We also symmetrize whenever more than one $\nabla_{\alpha}$ is involved in a single term, to account for the fact that the $\nabla_{\alpha}$ do not commute amongst themselves.

We first establish two useful conventions and recall some facts. Once a map $\Omega_{j}$ has been defined as above, we shall immediately extend it to be a left $\mathbb{C}[\mathfrak{n}]$-linear map from $\mathbb{C}[\mathfrak{n}] \otimes \operatorname{Dom}\left(\Omega_{j}\right)$ to $\mathbb{D}[\mathfrak{n}]$. Throughout this section, we shall write $X=\sum_{\nu \in R\left(V^{+}\right)} \xi_{\nu} X_{\nu} \in \mathbb{C}[\mathfrak{n}] \otimes \mathfrak{n}$ for the generic element of $V^{+}$. If $Z \in \mathfrak{l}$ satisfies $\lambda_{\chi}(Z)=0$ then Lemma 2.7 implies that

$$
N_{\beta, \beta^{\prime}}^{-1} M_{\beta^{\prime} \alpha}(Z)=N_{\alpha, \alpha^{\prime}}^{-1} M_{\alpha^{\prime} \beta}(Z)
$$

for all $\alpha, \beta \in R\left(V^{+}\right)$. We shall make repeated use of this identity below. From Lemma 2.4, $\left[\tau_{2}(X), X_{\gamma}\right]=0$ and so $\lambda_{\chi}\left(\tau_{2}(X)\right)=0$. Thus (5.1) applies in particular to $Z=\tau_{2}(X)$.

The map $\Omega_{1}: V^{+} \rightarrow \mathbb{D}[\mathfrak{n}]$ is to be modeled on the map

$$
Y \mapsto\left(X \mapsto \mathbf{B}_{\mathfrak{g}}\left(\tau_{1}(X), Y\right)\right)
$$


by the procedure described above. Now $\mathbf{B}_{\mathfrak{g}}\left(\tau_{1}(X), Y\right)=-\langle X, Y\rangle$ and this leads us to define

$$
\Omega_{1}(Y)=\sum_{\alpha \in R\left(V^{+}\right)} N_{\alpha, \alpha^{\prime}}^{-1}\left\langle Y, X_{\alpha^{\prime}}\right\rangle \nabla_{\alpha}
$$

Note that $\Omega_{1}\left(X_{\beta}\right)=\nabla_{\beta}$, so that $\Omega_{1}$ is simply the left $\mathbb{C}[\mathfrak{n}]$-linear extension of the map $\mathbf{r}: V^{+} \rightarrow \mathbb{D}[\mathfrak{n}]$ defined in Section 4 . The reason for introducing this redundant notation is to emphasize the uniformity between the various $\Omega_{j}$.

Theorem 5.1. For all $Y \in V^{+}$we have

$$
\left[D_{-\gamma}, \Omega_{1}(Y)\right]=-\xi_{\gamma} \Omega_{1}(Y)+\Omega_{1}\left(\left[\tau_{2}(X), Y\right]\right)+s\langle Y, X\rangle .
$$

Proof. For $Y=X_{\alpha}$, the proposed identity follows from Theorem 4.1. Since both sides are linear in $Y$, this is sufficient.

Next we define a map $\Omega_{2}: \mathfrak{l} \rightarrow \mathbb{D}[\mathfrak{n}]$ modeled on the map $\mathfrak{l} \rightarrow \mathbb{C}[\mathfrak{n}]$ given by

$$
Z \mapsto\left(X \mapsto \mathbf{B}_{\mathfrak{g}}\left(\tau_{2}(X), Z\right)\right) .
$$

A computation reveals that

$$
\mathbf{B}_{\mathfrak{g}}\left(\tau_{2}(X), Z\right)=-\frac{1}{2} \sum_{\alpha, \beta \in R\left(V^{+}\right)} N_{\alpha, \alpha^{\prime}} M_{\beta \alpha^{\prime}}(Z) \xi_{\alpha} \xi_{\beta},
$$

and this leads us to define

$$
\Omega_{2}(Z)=\frac{1}{2} \sum_{\alpha, \beta \in R\left(V^{+}\right)} N_{\beta, \beta^{\prime}}^{-1} M_{\beta^{\prime} \alpha}(Z)\left\ulcorner\nabla_{\alpha} \nabla_{\beta}\right\urcorner,
$$

where $\left\ulcorner\nabla_{\alpha} \nabla_{\beta}\right\urcorner=(1 / 2)\left(\nabla_{\alpha} \nabla_{\beta}+\nabla_{\beta} \nabla_{\alpha}\right)$. By using the transformation law for $\tau_{2}$ and the invariance of $\mathbf{B}_{\mathfrak{g}}$, one verifies that

$$
\mathbf{B}_{\mathfrak{g}}\left(\tau_{2}(X), \operatorname{Ad}(l) Z\right)=\chi(l) \mathbf{B}_{\mathfrak{g}}\left(\tau_{2}\left(\operatorname{Ad}\left(l^{-1}\right) X\right), Z\right) .
$$

It follows from this, on taking account of the dualization in passing from the original map to $\Omega_{2}$, that $\Omega_{2}(\operatorname{Ad}(l) Z)=\chi(l)^{-1} l \cdot \Omega_{2}(Z)$ for all $Z \in \mathfrak{l}$ and $l \in L$.

Observe that $\Omega_{2}\left(H_{\gamma}\right)=0$. For this reason, we focus our attention on those $Z$ that lie in the kernel of $\lambda_{\chi}$. For such $Z$, it a consequence of (5.1) that the symmetrization in the definition of $\Omega_{2}(Z)$ is unnecessary. Thus, for 
$Z \in \operatorname{ker}\left(\lambda_{\chi}\right)$

$$
\begin{aligned}
\Omega_{2}(Z) & =\frac{1}{2} \sum_{\alpha, \beta \in R\left(V^{+}\right)} N_{\beta, \beta^{\prime}}^{-1} M_{\beta^{\prime} \alpha}(Z) \nabla_{\beta} \nabla_{\alpha} \\
& =\frac{1}{2} \sum_{\alpha, \beta \in R\left(V^{+}\right)} N_{\beta, \beta^{\prime}}^{-1} M_{\beta^{\prime} \alpha}(Z) \Omega_{1}\left(X_{\beta}\right) \Omega_{1}\left(X_{\alpha}\right) \\
& =\frac{1}{2} \sum_{\beta \in R\left(V^{+}\right)} N_{\beta, \beta^{\prime}}^{-1} \Omega_{1}\left(X_{\beta}\right) \Omega_{1}\left(\left[Z, X_{\beta^{\prime}}\right]\right) .
\end{aligned}
$$

For later use, we note the alternate expression

$$
\Omega_{2}(Z)=-\frac{1}{2} \sum_{\alpha \in R\left(V^{+}\right)} N_{\alpha, \alpha^{\prime}}^{-1} \Omega_{1}\left(\left[Z, X_{\alpha}\right]\right) \Omega_{1}\left(X_{\alpha^{\prime}}\right)
$$

which may be similarly obtained.

Theorem 5.2. $\quad$ Let $\mathfrak{C}$ be a component of $\mathcal{D}_{\gamma}(\mathfrak{g}, \mathfrak{h})$. For all $Z \in \mathfrak{l}(\mathfrak{C})$ we have

$$
\left[D_{-\gamma}, \Omega_{2}(Z)\right]=-2 \xi_{\gamma} \Omega_{2}(Z)+\Omega_{2}\left(\left[\tau_{2}(X), Z\right]\right)+\left(s-s_{2}\right) \Omega_{1}([Z, X]),
$$

with $s_{2}=(1 / 2) c(\mathfrak{g}, \mathcal{C})-1$.

Proof. Note that $\mathfrak{l}(\mathcal{C})$ is contained in the kernel of $\lambda_{\chi}$. The proposed identity is linear in $Z$ and one may verify, using the transformation laws that have been given for the various objects involved, that

$$
\left[D_{-\gamma}, \Omega_{2}(\operatorname{Ad}(l) Z)\right]=l \cdot\left[D_{-\gamma}, \Omega_{2}(Z)\right],
$$

and that the right-hand side enjoys the same transformation law under $L$. The set $\operatorname{Ad}(L)(\mathfrak{h} \cap \mathfrak{l}(\mathfrak{C}))$ is dense in $\mathfrak{l}(\mathfrak{C})$ and it follows that it suffices to establish the identity for $Z \in \mathfrak{h} \cap \mathfrak{l}(\mathcal{C})$. Since this set is spanned by $\left\{H_{\lambda} \mid \lambda \in R(\mathfrak{l}, \mathcal{C})\right\}$, it in fact suffices to establish the identity with $Z=H_{\lambda}$. Under this assumption we have

$$
\Omega_{2}(Z)=\frac{1}{2} \sum_{\beta} N_{\beta, \beta^{\prime}}^{-1} \beta^{\prime}(Z) \Omega_{1}\left(X_{\beta}\right) \Omega_{1}\left(X_{\beta^{\prime}}\right),
$$

where the sum is understood to run over $R\left(V^{+}\right)$. We shall use $Z$ and $H_{\lambda}$ interchangeably below, since the precise identity of $Z$ is often irrelevant. It follows that

$$
\left[D_{-\gamma}, \Omega_{2}(Z)\right]=\frac{1}{2} \sum_{\beta} N_{\beta, \beta^{\prime}}^{-1} \beta^{\prime}(Z)\left[D_{-\gamma}, \Omega_{1}\left(X_{\beta}\right) \Omega_{1}\left(X_{\beta^{\prime}}\right)\right]
$$


and, by Theorem 5.1,

$$
\begin{aligned}
& {\left[D_{-\gamma}, \Omega_{1}\left(X_{\beta}\right) \Omega_{1}\left(X_{\beta^{\prime}}\right)\right]} \\
& =\left[D_{-\gamma}, \Omega_{1}\left(X_{\beta}\right)\right] \Omega_{1}\left(X_{\beta^{\prime}}\right)+\Omega_{1}\left(X_{\beta}\right)\left[D_{-\gamma}, \Omega_{1}\left(X_{\beta^{\prime}}\right)\right] \\
& =\left(-\xi_{\gamma} \Omega_{1}\left(X_{\beta}\right)+\Omega_{1}\left(\left[\tau_{2}(X), X_{\beta}\right]\right)+s\left\langle X_{\beta}, X\right\rangle\right) \Omega_{1}\left(X_{\beta^{\prime}}\right) \\
& +\Omega_{1}\left(X_{\beta}\right)\left(-\xi_{\gamma} \Omega_{1}\left(X_{\beta^{\prime}}\right)+\Omega_{1}\left(\left[\tau_{2}(X), X_{\beta^{\prime}}\right]\right)+s\left\langle X_{\beta^{\prime}}, X\right\rangle\right) .
\end{aligned}
$$

Our task now is to determine the contribution made when each term in this expression is substituted back into (5.2).

First,

$$
\begin{aligned}
\Omega_{1}\left(X_{\beta}\right) \xi_{\gamma} \Omega_{1}\left(X_{\beta^{\prime}}\right) & =\xi_{\gamma} \Omega_{1}\left(X_{\beta}\right) \Omega_{1}\left(X_{\beta^{\prime}}\right)+\left(\Omega_{1}\left(X_{\beta}\right) \bullet \xi_{\gamma}\right) \Omega_{1}\left(X_{\beta^{\prime}}\right) \\
& =\xi_{\gamma} \Omega_{1}\left(X_{\beta}\right) \Omega_{1}\left(X_{\beta^{\prime}}\right)-(1 / 2) N_{\beta, \beta^{\prime}} \xi_{\beta^{\prime}} \Omega_{1}\left(X_{\beta^{\prime}}\right)
\end{aligned}
$$

and so the contribution from this term and $-\xi_{\gamma} \Omega_{1}\left(X_{\beta}\right) \Omega_{1}\left(X_{\beta^{\prime}}\right)$ is

$$
\begin{aligned}
& \frac{1}{2} \sum_{\beta} N_{\beta, \beta^{\prime}}^{-1} \beta^{\prime}(Z)\left(-2 \xi_{\gamma} \Omega_{1}\left(X_{\beta}\right) \Omega_{1}\left(X_{\beta^{\prime}}\right)+(1 / 2) N_{\beta, \beta^{\prime}} \xi_{\beta^{\prime}} \Omega_{1}\left(X_{\beta^{\prime}}\right)\right) \\
& =-2 \xi_{\gamma} \Omega_{2}(Z)+\frac{1}{4} \sum_{\beta} \beta^{\prime}(Z) \xi_{\beta^{\prime}} \Omega_{1}\left(X_{\beta^{\prime}}\right) \\
& =-2 \xi_{\gamma} \Omega_{2}(Z)+(1 / 4) \Omega_{1}([Z, X]) .
\end{aligned}
$$

Secondly, we consider the contribution from the terms containing $s$. It is

$$
\begin{aligned}
& \frac{1}{2} s \sum_{\beta} N_{\beta, \beta^{\prime}}^{-1} \beta^{\prime}(Z)\left(N_{\beta, \beta^{\prime}} \xi_{\beta^{\prime}} \Omega_{1}\left(X_{\beta^{\prime}}\right)+\Omega_{1}\left(X_{\beta}\right) N_{\beta^{\prime}, \beta} \xi_{\beta}\right) \\
& =\frac{1}{2} s \sum_{\beta} \beta^{\prime}(Z)\left(\xi_{\beta^{\prime}} \Omega_{1}\left(X_{\beta^{\prime}}\right)-\Omega_{1}\left(X_{\beta}\right) \xi_{\beta}\right) \\
& =\frac{1}{2} s \sum_{\beta} \beta^{\prime}(Z)\left(\xi_{\beta^{\prime}} \Omega_{1}\left(X_{\beta^{\prime}}\right)-\xi_{\beta} \Omega_{1}\left(X_{\beta}\right)-1\right) \\
& =\frac{1}{2} s \sum_{\beta} \beta^{\prime}(Z) \xi_{\beta^{\prime}} \Omega_{1}\left(X_{\beta^{\prime}}\right)+\frac{1}{2} s \sum_{\beta} \beta(Z) \xi_{\beta} \Omega_{1}\left(X_{\beta}\right)-\frac{1}{2} s \sum_{\beta} \beta^{\prime}(Z) \\
& =s \Omega_{1}([Z, X]),
\end{aligned}
$$

where we have used the facts that $\beta(Z)=-\beta^{\prime}(Z)$ and $\sum_{\beta} \beta^{\prime}(Z)=0$, both of which are true because $\gamma(Z)=0$.

Before determining the contribution from the remaining terms, we derive an alternate expression for $\Omega_{2}\left(\left[\tau_{2}, Z\right]\right)$. It is easy to verify that we have 
$M_{\alpha \beta}\left(\left[\tau_{2}(X), Z\right]\right)=(\alpha(Z)-\beta(Z)) M_{\alpha \beta}\left(\tau_{2}(X)\right)$ for all $\alpha, \beta \in R\left(V^{+}\right)$and this identity will be used in the derivation. Equation (5.1) will also be required, and we choose to include the (optional) symmetrization in the definition of $\Omega_{2}$ in this case. We have

$$
\begin{aligned}
\Omega_{2}\left(\left[\tau_{2}(X), Z\right]\right)= & \frac{1}{2} \sum_{\mu, \nu} N_{\mu, \mu^{\prime}}^{-1} M_{\mu^{\prime} \nu}\left(\left[\tau_{2}(X), Z\right]\right)\left\ulcorner\Omega_{1}\left(X_{\mu}\right) \Omega_{1}\left(X_{\nu}\right)\right\urcorner \\
& =\frac{1}{2} \sum_{\mu, \nu} N_{\mu, \mu^{\prime}}^{-1}\left(\mu^{\prime}(Z)-\nu(Z)\right) M_{\mu^{\prime} \nu}\left(\tau_{2}(X)\right)\left\ulcorner\Omega_{1}\left(X_{\mu}\right) \Omega_{1}\left(X_{\nu}\right)\right\urcorner \\
& =\frac{1}{2} \sum_{\mu, \nu} N_{\mu, \mu^{\prime}}^{-1} \mu^{\prime}(Z) M_{\mu^{\prime} \nu}\left(\tau_{2}(X)\right)\left\ulcorner\Omega_{1}\left(X_{\mu}\right) \Omega_{1}\left(X_{\nu}\right)\right\urcorner \\
& +\frac{1}{2} \sum_{\mu, \nu} N_{\nu, \nu^{\prime}}^{-1} \nu^{\prime}(Z) M_{\nu^{\prime} \mu}\left(\tau_{2}(X)\right)\left\ulcorner\Omega_{1}\left(X_{\mu}\right) \Omega_{1}\left(X_{\nu}\right)\right\urcorner \\
& =\sum_{\mu, \nu} N_{\mu, \mu^{\prime}}^{-1} \mu^{\prime}(Z) M_{\mu^{\prime} \nu}\left(\tau_{2}(X)\right)\left\ulcorner\Omega_{1}\left(X_{\mu}\right) \Omega_{1}\left(X_{\nu}\right)\right\urcorner .
\end{aligned}
$$

We are now ready to tackle the contribution from the remaining terms, which is

$$
\begin{aligned}
& \frac{1}{2} \sum_{\beta} N_{\beta, \beta^{\prime}}^{-1} \beta^{\prime}(Z)\left(\Omega_{1}\left(\left[\tau_{2}(X), X_{\beta}\right]\right) \Omega_{1}\left(X_{\beta^{\prime}}\right)+\Omega_{1}\left(X_{\beta}\right) \Omega_{1}\left(\left[\tau_{2}(X), X_{\beta^{\prime}}\right]\right)\right) \\
& =\frac{1}{2} \sum_{\beta, \mu} N_{\beta, \beta^{\prime}}^{-1} \beta^{\prime}(Z) M_{\beta \mu}\left(\tau_{2}(X)\right) \Omega_{1}\left(X_{\mu}\right) \Omega_{1}\left(X_{\beta^{\prime}}\right) \\
& +\frac{1}{2} \sum_{\beta, \mu} N_{\beta, \beta^{\prime}}^{-1} \beta^{\prime}(Z) \Omega_{1}\left(X_{\beta}\right) M_{\beta^{\prime} \mu}\left(\tau_{2}(X)\right) \Omega_{1}\left(X_{\mu}\right) \\
& =\frac{1}{2} \sum_{\beta, \mu} N_{\beta, \beta^{\prime}}^{-1} \beta^{\prime}(Z) M_{\beta \mu}\left(\tau_{2}(X)\right) \Omega_{1}\left(X_{\mu}\right) \Omega_{1}\left(X_{\beta^{\prime}}\right) \\
& +\frac{1}{2} \sum_{\beta, \mu} N_{\beta, \beta^{\prime}}^{-1} \beta^{\prime}(Z) \Omega_{1}\left(X_{\beta^{\prime}}\right) M_{\beta \mu}\left(\tau_{2}(X)\right) \Omega_{1}\left(X_{\mu}\right) \\
& =\sum_{\beta, \mu} N_{\beta, \beta^{\prime}}^{-1}, \beta^{\prime}(Z) M_{\beta \mu}\left(\tau_{2}(X)\right)\left\ulcorner\Omega_{1}\left(X_{\mu}\right) \Omega_{1}\left(X_{\beta^{\prime}}\right)\right\urcorner \\
& +\frac{1}{2} \sum_{\beta, \mu} N_{\beta, \beta^{\prime}}^{-1}, \beta^{\prime}(Z)\left(\Omega_{1}\left(X_{\beta^{\prime}}\right) \bullet M_{\beta \mu}\left(\tau_{2}(X)\right)\right) \Omega_{1}\left(X_{\mu}\right) \\
& =\sum_{\beta, \mu} N_{\beta, \beta^{\prime}}^{-1}, \beta^{\prime}(Z) M_{\beta^{\prime} \mu}\left(\tau_{2}(X)\right)\left\ulcorner\Omega_{1}\left(X_{\mu}\right) \Omega_{1}\left(X_{\beta}\right)\right\urcorner \\
& +\frac{1}{2} \sum_{\beta, \mu} N_{\beta, \beta^{\prime}}^{-1} \beta^{\prime}(Z)\left(\Omega_{1}\left(X_{\beta}\right) \bullet M_{\beta^{\prime} \mu}\left(\tau_{2}(X)\right)\right) \Omega_{1}\left(X_{\mu}\right)
\end{aligned}
$$




$$
\begin{aligned}
& =\Omega_{2}\left(\left[\tau_{2}(X), Z\right]\right)+\frac{1}{2} \sum_{\beta, \mu} N_{\beta, \beta^{\prime}}^{-1} \beta^{\prime}(Z) M_{\beta^{\prime} \mu}\left(\partial_{\beta} \bullet \tau_{2}(X)\right) \Omega_{1}\left(X_{\mu}\right) \\
& =\Omega_{2}\left(\left[\tau_{2}(X), Z\right]\right)+\frac{1}{2} \sum_{\beta} N_{\beta, \beta^{\prime}}^{-1} \beta^{\prime}(Z) \Omega_{1}\left(\left[\partial_{\beta} \bullet \tau_{2}(X), X_{\beta^{\prime}}\right]\right) .
\end{aligned}
$$

For brevity, let us refer to the second term above as $T$. Lemma 3.6 and the Jacobi identity give

$$
\begin{aligned}
& {\left[\partial_{\beta} \bullet \tau_{2}(X), X_{\beta^{\prime}}\right]} \\
& =(1 / 2)\left\langle X_{\beta}, X\right\rangle\left[H_{\gamma}, X_{\beta^{\prime}}\right]+N_{\beta,-\gamma}\left[\left[X, X_{-\beta^{\prime}}\right], X_{\beta^{\prime}}\right] \\
& =(1 / 2)\left\langle X_{\beta}, X\right\rangle X_{\beta^{\prime}}+N_{\beta,-\gamma}\left[\left[X, X \beta_{-\beta^{\prime}}\right], X_{\beta^{\prime}}\right] \\
& =(1 / 2) N_{\beta, \beta^{\prime}} \xi_{\beta^{\prime}} X_{\beta^{\prime}}+N_{\beta,-\gamma}\left[\left[X, X_{-\beta^{\prime}}\right], X_{\beta^{\prime}}\right] \\
& =(1 / 2) N_{\beta, \beta^{\prime}} \xi_{\beta^{\prime}} X_{\beta^{\prime}}+N_{\beta,-\gamma}\left[X,\left[X_{-\beta^{\prime}}, X_{\beta^{\prime}}\right]\right]-N_{\beta,-\gamma}\left[X_{-\beta^{\prime}},\left[X, X_{\beta^{\prime}}\right]\right] \\
& =(1 / 2) N_{\beta, \beta^{\prime}} \xi_{\beta^{\prime}} X_{\beta^{\prime}}+N_{\beta,-\gamma}\left[H_{\beta^{\prime}}, X\right]-N_{\beta,-\gamma} N_{\beta, \beta^{\prime}} \xi_{\beta}\left[X_{-\beta^{\prime}}, X_{\gamma}\right] \\
& =(1 / 2) N_{\beta, \beta^{\prime}} \xi_{\beta^{\prime}} X_{\beta^{\prime}}+N_{\beta,-\gamma}\left[H_{\beta^{\prime}}, X\right]-N_{\beta,-\gamma} N_{\beta, \beta^{\prime}} N_{-\beta^{\prime}, \gamma} \xi_{\beta} X_{\beta} \\
& =(1 / 2) N_{\beta, \beta^{\prime}} \xi_{\beta^{\prime}} X_{\beta^{\prime}}+N_{\beta,-\gamma}\left[H_{\beta^{\prime}}, X\right]+N_{\beta, \beta^{\prime}} N_{\beta,-\gamma} N_{\beta^{\prime},-\gamma} \xi_{\beta} X_{\beta} \\
& =(1 / 2) N_{\beta, \beta^{\prime}} \xi_{\beta^{\prime}} X_{\beta^{\prime}}+N_{\beta,-\gamma}\left[H_{\beta^{\prime}}, X\right]-(1 / 4)\|\beta\|^{4} N_{\beta, \beta^{\prime}}^{3} \xi_{\beta} X_{\beta} \\
& =(1 / 2) N_{\beta, \beta^{\prime}} \xi_{\beta^{\prime}} X_{\beta^{\prime}}+N_{\beta,-\gamma}\left[H_{\beta^{\prime}}, X\right]-N_{\beta, \beta^{\prime}} \xi_{\beta} X_{\beta} .
\end{aligned}
$$

By substituting this into the above expression for $T$ we obtain

$$
\begin{aligned}
T & =(3 / 4) \Omega_{1}([Z, X])+\frac{1}{2} \sum_{\beta} N_{\beta, \beta^{\prime}}^{-1} \beta^{\prime}(Z) N_{\beta,-\gamma} \Omega_{1}\left(\left[H_{\beta^{\prime}}, X\right]\right) \\
& =(3 / 4) \Omega_{1}([Z, X])-\frac{1}{4} \sum_{\beta}\|\beta\|^{2} \beta^{\prime}(Z) \Omega_{1}\left(\left[H_{\beta^{\prime}}, X\right]\right) .
\end{aligned}
$$

In order to complete the proof, it remains to show that the second summand in this expression is equal to $-(1 / 2) c(\mathfrak{g}, \mathcal{C}) \Omega_{1}([Z, X])$. Recall that $Z=H_{\lambda}$ with $\lambda \in R(\mathfrak{l}, \mathrm{C})$. We have

$$
\begin{aligned}
& -\frac{1}{4} \sum_{\beta}\|\beta\|^{2} \beta^{\prime}\left(H_{\lambda}\right) \Omega_{1}\left(\left[H_{\beta^{\prime}}, X\right]\right) \\
& =-\frac{1}{4} \sum_{\beta, \nu}\|\beta\|^{2} \beta^{\prime}\left(H_{\lambda}\right) \xi_{\nu} \Omega_{1}\left(\left[H_{\beta^{\prime}}, X_{\nu}\right]\right) \\
& =-\frac{1}{4} \sum_{\beta, \nu}\|\beta\|^{2} \beta^{\prime}\left(H_{\lambda}\right) \nu\left(H_{\beta^{\prime}}\right) \xi_{\nu} \Omega_{1}\left(X_{\nu}\right) \\
& =-\frac{1}{\|\lambda\|^{2}} \sum_{\nu}\left(\sum_{\beta}\left(\nu, \beta^{\prime}\right)\left(\beta^{\prime}, \lambda\right)\right) \xi_{\nu} \Omega_{1}\left(X_{\nu}\right)
\end{aligned}
$$




$$
\begin{aligned}
& =-\frac{c(\mathfrak{g}, \mathfrak{C})}{\|\lambda\|^{2}} \sum_{\nu}(\nu, \lambda) \xi_{\nu} \Omega_{1}\left(X_{\nu}\right) \\
& =-\frac{1}{2} c(\mathfrak{g}, \mathcal{C}) \sum_{\nu} \nu\left(H_{\lambda}\right) \xi_{\nu} \Omega_{1}\left(X_{\nu}\right) \\
& =-(1 / 2) c(\mathfrak{g}, \mathfrak{C}) \Omega_{1}\left(\left[H_{\lambda}, X\right]\right) .
\end{aligned}
$$

The critical step, from line four to line five, was made with the aid of Proposition 2.1. This completes the proof.

Theorem 5.2 does not exhaust the conformally invariant systems that can be derived from $\Omega_{2}$. When $\mathfrak{g}$ is of type $\mathrm{A}_{r}$, there is an additional conformally invariant differential operator on $\mathfrak{n}$ associated with $\Omega_{2}$. It appears because, in this case, the span of $H_{\gamma}$ does not exhaust the center of $\mathfrak{l}$. We shall now complete our discussion of $\Omega_{2}$ by describing this operator.

For the remainder of this section, we shall assume that $\mathfrak{g}=\mathfrak{s l}(r+1)$ and use the standard matrices $E_{i j}$ to describe elements of this algebra. We shall take $\mathfrak{q}$ to be the standard block-upper-triangular parabolic subalgebra of $\mathfrak{g}$ corresponding to the partition $(1, r-1,1)$ of $r+1$. The root system will be described using the standard picture inside $\mathbb{R}^{r+1}$ in which $R^{+}=\left\{e_{i}-e_{j} \mid 1 \leq\right.$ $i<j \leq r+1\}$. Then $\gamma=e_{1}-e_{r+1}$ and $R\left(V^{+}\right)=\left\{\beta_{j}, \beta_{j}^{\prime} \mid 1 \leq j \leq(r-1)\right\}$ with $\beta_{j}=e_{1}-e_{j+1}$. For brevity, we write $X_{j}$ for $X_{\beta_{j}}=E_{1, j+1}$ and $X_{j}^{\prime}$ for $X_{\beta_{j}^{\prime}}=E_{j+1, r+1}$. We take $X_{\gamma}=E_{1, r+1}$ so that $N_{\beta_{j}, \beta_{j}^{\prime}}=1$ for all $j$. Let

$$
Z_{0}=\frac{2}{r+1}\left(E_{22}+\cdots+E_{r r}-\frac{r-1}{2}\left(E_{11}+E_{r+1, r+1}\right)\right) \in \mathfrak{g} .
$$

This element lies in the center of $\mathfrak{l}$ and $\lambda_{\chi}\left(Z_{0}\right)=0$. The normalization has been chosen so that $\left[Z_{0}, X_{j}\right]=-X_{j}$ and $\left[Z_{0}, X_{j}^{\prime}\right]=X_{j}^{\prime}$ for all $1 \leq j \leq(r-1)$. It follows that

$$
\Omega_{2}\left(Z_{0}\right)=\sum_{j=1}^{r-1}\left\ulcorner\Omega_{1}\left(X_{j}\right) \Omega_{1}\left(X_{j}^{\prime}\right)\right\urcorner,
$$

which may also be expressed as

$$
\Omega_{2}\left(Z_{0}\right)=\sum_{j=1}^{r-1} \Omega_{1}\left(X_{j}\right) \Omega_{1}\left(X_{j}^{\prime}\right)-\frac{r-1}{2} \partial_{\gamma} .
$$

Theorem 5.3. We have

$$
\left[D_{-\gamma}, \Omega_{2}\left(Z_{0}\right)\right]=-2 \xi_{\gamma} \Omega_{2}\left(Z_{0}\right)+\left(s-\frac{r-1}{2}\right) \Omega_{1}\left(\left[Z_{0}, X\right]\right) .
$$


Proof. The majority of the computations made in the proof of Theorem 5.2 remain valid here, and it is routine to modify the others to complete the proof. However, it is perhaps easier to compute the commutator from scratch using the special features of the current situation. If we let $\xi_{j}$ and $\xi_{j}^{\prime}$ be the coordinates dual to $X_{j}$ and $X_{j}^{\prime}$, respectively, and define

$$
\Delta_{0}=\frac{1}{2} \sum_{j=1}^{r-1} \xi_{j} \xi_{j}^{\prime}
$$

then

$$
\tau_{2}(X)=\Delta_{0}(X)\left(E_{11}+E_{r+1, r+1}\right)-\sum_{i, j=1}^{r-1} \xi_{i}^{\prime} \xi_{j} E_{i+1, j+1} .
$$

This gives

$$
\begin{aligned}
& {\left[\tau_{2}(X), X_{j}\right]=\Delta_{0}(X) X_{j}+\xi_{j}^{\prime} \sum_{i=1}^{r-1} \xi_{i} X_{i},} \\
& {\left[\tau_{2}(X), X_{j}^{\prime}\right]=-\Delta_{0}(X) X_{j}^{\prime}-\xi_{j} \sum_{i=1}^{r-1} \xi_{i}^{\prime} X_{i}^{\prime},}
\end{aligned}
$$

and makes it easy to obtain explicit expressions for both $\left[D_{-\gamma}, \Omega_{1}\left(X_{j}\right)\right]$ and $\left[D_{-\gamma}, \Omega_{1}\left(X_{j}^{\prime}\right)\right]$ from Theorem 5.1 . The computation is easily completed from this point.

\section{§6. Conformally Invariant Systems II}

In this section, we assume that the algebra $\mathfrak{g}$ is not of type $\mathrm{C}_{r}$, so that the covariants $\tau_{3}$ and $\tau_{4}$ are not identically zero. Simply put, our aim is to construct maps

$$
\begin{aligned}
& \Omega_{3}: V^{-} \rightarrow \mathbb{D}[\mathfrak{n}], \\
& \Omega_{4}: \mathfrak{g}_{-\gamma} \rightarrow \mathbb{D}[\mathfrak{n}],
\end{aligned}
$$

corresponding to these covariants. However, two complications will arise, one related to the fact that $\mathcal{D}_{\gamma}(\mathfrak{g}, \mathfrak{h})$ may fail to be connected, and the other to the need for "correction terms" to secure conformal invariance. Because of the first complication, we shall only construct $\Omega_{3}$ when $\mathfrak{g}$ is an exceptional algebra. In the exceptional cases, $\Omega_{3}$ is essential to reach our overall goal, whereas in the classical cases it is not. To specify $\Omega_{4}$ it suffices to give the element $\Omega_{4}\left(X_{-\gamma}\right)$ and this is what we shall do. 
Let us first establish some further notation. We extend the symmetrization operator used above by setting

$$
\ulcorner a b c\urcorner=(1 / 6)(a b c+a c b+b a c+b c a+c a b+c b a)
$$

and similarly for more than three factors. To determine which symmetrization operator is meant, one looks at the number of factors at the topmost level: thus, for example, $\ulcorner(a b) c\urcorner=(1 / 2)(a b c+c a b)$, since the expression $(a b) c$ has two factors at the topmost level.

The algebra $\mathfrak{l}$ decomposes as $\mathfrak{l}=\mathbb{C} H_{\gamma} \oplus \operatorname{ker}\left(\lambda_{\chi}\right)$ and we write $\operatorname{pr}_{0}: \mathfrak{l} \rightarrow$ $\operatorname{ker}\left(\lambda_{\chi}\right)$ for the projection onto the second summand. Explicitly, we have

$$
\operatorname{pr}_{0}(Z)=Z-(1 / 2) \lambda_{\chi}(Z) H_{\gamma}
$$

Because $\Omega_{2}\left(H_{\gamma}\right)=0$, we have $\Omega_{2}(Z)=\Omega_{2}\left(\operatorname{pr}_{0}(Z)\right)$ for all $Z \in \mathfrak{l}$. If $\mathfrak{g}$ is not of type $\mathrm{A}_{r}$ then $\operatorname{ker}\left(\lambda_{\chi}\right)$ is the direct sum of $\mathfrak{l}(\mathcal{C})$ over all components $\mathcal{C}$ of $\mathcal{D}_{\gamma}(\mathfrak{g}, \mathfrak{h})$. If $\mathfrak{g}$ is of type $\mathrm{A}_{r}$ then $\mathcal{D}_{\gamma}(\mathfrak{g}, \mathfrak{h})$ has a single component and $\operatorname{ker}\left(\lambda_{\chi}\right)=\mathbb{C} Z_{0} \oplus \mathfrak{l}(\mathcal{C})$, where $Z_{0}$ is the element introduced at the end of Section 5 . For these reasons, Theorems 5.2 and 5.3 between them serve to determine all possible commutators $\left[D_{-\gamma}, \Omega_{2}(Z)\right]$ for $Z \in \mathfrak{l}$.

For $Y \in V^{-}$we have

$$
\mathbf{B}_{\mathfrak{g}}\left(\tau_{3}(X), Y\right)=\frac{1}{6} \sum_{\alpha, \beta, \epsilon \in R\left(V^{+}\right)} N_{\alpha, \alpha^{\prime}} M_{\beta \alpha^{\prime}}\left(\left[X_{\epsilon}, Y\right]\right) \xi_{\alpha} \xi_{\beta} \xi_{\epsilon}
$$

and the yoga explained earlier leads us to define

$$
\Omega_{3}^{\prime}(Y)=-\frac{1}{6} \sum_{\alpha, \beta, \epsilon \in R\left(V^{+}\right)} N_{\beta, \beta^{\prime}}^{-1} N_{\epsilon, \epsilon^{\prime}}^{-1} M_{\beta^{\prime} \alpha}\left(\left[X_{\epsilon^{\prime}}, Y\right]\right)\left\ulcorner\nabla_{\alpha} \nabla_{\beta} \nabla_{\epsilon}\right\urcorner .
$$

As with $\Omega_{2}$, one verifies that $\Omega_{3}^{\prime}(\operatorname{Ad}(l) Y)=\chi(l)^{-2} l \cdot \Omega_{3}^{\prime}(Y)$ for all $l \in L$ and $Y \in V^{-}$. Our first task will be to obtain a more tractable expression for $\Omega_{3}^{\prime}(Y)$. We remark that the nature of the correction term mentioned above will be revealed as a byproduct of doing this.

Lemma 6.1. For $\alpha, \beta, \epsilon \in R\left(V^{+}\right)$we have

$$
\left\ulcorner\nabla_{\alpha} \nabla_{\beta} \nabla_{\epsilon}\right\urcorner=\left\ulcorner\left(\nabla_{\alpha} \nabla_{\beta}\right) \nabla_{\epsilon}\right\urcorner-(1 / 2) \kappa_{\alpha \beta^{\prime}} N_{\alpha, \alpha^{\prime}} \nabla_{\epsilon} \partial_{\gamma}
$$

Proof. For notational compactness, let us write $\nabla_{\alpha \beta \epsilon}$ for $\nabla_{\alpha} \nabla_{\beta} \nabla_{\epsilon}$. Note 
that $\left[\nabla_{\mu}, \nabla_{\nu}\right]=\kappa_{\mu \nu^{\prime}} N_{\mu, \mu^{\prime}} \partial_{\gamma}$ and that $\partial_{\gamma}$ commutes with all $\nabla_{\alpha}$. Thus

$$
\begin{aligned}
& \left\ulcorner\nabla_{\alpha} \nabla_{\beta} \nabla_{\epsilon}\right\urcorner \\
& =(1 / 6)\left(\nabla_{\alpha \beta \epsilon}+\nabla_{\alpha \epsilon \beta}+\nabla_{\beta \alpha \epsilon}+\nabla_{\beta \epsilon \alpha}+\nabla_{\epsilon \alpha \beta}+\nabla_{\epsilon \beta \alpha}\right) \\
& =(1 / 6)\left(\nabla_{\alpha \beta \epsilon}+\nabla_{\alpha \beta \epsilon}+\nabla_{\alpha}\left[\nabla_{\epsilon}, \nabla_{\beta}\right]+\nabla_{\beta \alpha \epsilon}\right. \\
& \left.+\nabla_{\epsilon \beta \alpha}+\left[\nabla_{\beta}, \nabla_{\epsilon}\right] \nabla_{\alpha}+\nabla_{\epsilon \alpha \beta}+\nabla_{\epsilon \beta \alpha}\right) \\
& =(1 / 6)\left(2 \nabla_{\alpha \beta \epsilon}+\nabla_{\beta \alpha \epsilon}+\nabla_{\epsilon \alpha \beta}+2 \nabla_{\epsilon \beta \alpha}\right) \\
& =(1 / 6)\left(3 \nabla_{\alpha \beta \epsilon}+\left[\nabla_{\beta}, \nabla_{\alpha}\right] \nabla_{\epsilon}+3 \nabla_{\epsilon \alpha \beta}+2 \nabla_{\epsilon}\left[\nabla_{\beta}, \nabla_{\alpha}\right]\right) \\
& =\left\ulcorner\left(\nabla_{\alpha} \nabla_{\beta}\right) \nabla_{\epsilon}\right\urcorner-(1 / 2)\left[\nabla_{\alpha}, \nabla_{\beta}\right] \nabla_{\epsilon} \\
& =\left\ulcorner\left(\nabla_{\alpha} \nabla_{\beta}\right) \nabla_{\epsilon}\right\urcorner-(1 / 2) \kappa_{\alpha \beta^{\prime}} N_{\alpha, \alpha^{\prime}} \nabla_{\epsilon} \partial_{\gamma} \text {. }
\end{aligned}
$$

Lemma 6.2. We have $\sum_{\alpha \in R\left(V^{+}\right)} N_{\alpha, \alpha^{\prime}}^{-1} \nabla_{\alpha} \nabla_{\alpha^{\prime}}=(1 / 2) \operatorname{dim}\left(V^{+}\right) \partial_{\gamma}$.

Proof. Let the indicated sum be $S$. Then

$$
\begin{aligned}
S & =-\sum_{\alpha} N_{\alpha, \alpha^{\prime}}^{-1} \nabla_{\alpha^{\prime}} \nabla_{\alpha} \\
& =-\sum_{\alpha} N_{\alpha, \alpha^{\prime}}^{-1}\left(\nabla_{\alpha} \nabla_{\alpha^{\prime}}+\left[\nabla_{\alpha^{\prime}}, \nabla_{\alpha}\right]\right) \\
& =-S+\sum_{\alpha} \partial_{\gamma} \\
& =-S+\operatorname{dim}\left(V^{+}\right) \partial_{\gamma}
\end{aligned}
$$

and the evaluation follows.

Lemma 6.3. For $\epsilon \in R\left(V^{+}\right)$and $Y \in V^{-}$we have $\lambda_{\chi}\left(\left[X_{\epsilon}, Y\right]\right)=$ $\left\langle X_{\epsilon},\left[Y, X_{\gamma}\right]\right\rangle$.

Proof. Since $\left[X_{\epsilon}, X_{\gamma}\right]=0$,

$$
\lambda_{\chi}\left(\left[X_{\epsilon}, Y\right]\right) X_{\gamma}=\left[\left[X_{\epsilon}, Y\right], X_{\gamma}\right]=\left[X_{\epsilon},\left[Y, X_{\gamma}\right]\right]=\left\langle X_{\epsilon},\left[Y, X_{\gamma}\right]\right\rangle X_{\gamma} .
$$

Proposition 6.1. For all $Y \in V^{-}$we have

$$
\Omega_{3}^{\prime}(Y)=-\frac{1}{6} \sum_{\alpha, \beta, \epsilon \in R\left(V^{+}\right)} N_{\beta, \beta^{\prime}}^{-1} N_{\epsilon, \epsilon^{\prime}}^{-1} M_{\beta^{\prime} \alpha}\left(\operatorname{pr}_{0}\left[X_{\epsilon^{\prime}}, Y\right]\right)\left\ulcorner\nabla_{\alpha} \nabla_{\beta} \nabla_{\epsilon}\right\urcorner .
$$


Proof. It suffices to show that

$$
S=\sum_{\alpha, \beta, \epsilon} N_{\beta, \beta^{\prime}}^{-1} N_{\epsilon, \epsilon^{\prime}}^{-1} \lambda_{\chi}\left(\left[X_{\epsilon^{\prime}}, Y\right]\right) M_{\beta^{\prime} \alpha}\left(H_{\gamma}\right)\left\ulcorner\nabla_{\alpha} \nabla_{\beta} \nabla_{\epsilon}\right\urcorner
$$

is zero. Now $M\left(H_{\gamma}\right)$ is the identity matrix and so

$$
S=-\sum_{\alpha, \epsilon} N_{\alpha, \alpha^{\prime}}^{-1} N_{\epsilon, \epsilon^{\prime}}^{-1} \lambda_{\chi}\left(\left[X_{\epsilon^{\prime}}, Y\right]\right)\left\ulcorner\nabla_{\alpha} \nabla_{\alpha^{\prime}} \nabla_{\epsilon}\right\urcorner .
$$

Lemma 6.1 gives $\left\ulcorner\nabla_{\alpha} \nabla_{\alpha^{\prime}} \nabla_{\epsilon}\right\urcorner=\left\ulcorner\left(\nabla_{\alpha} \nabla_{\alpha^{\prime}}\right) \nabla_{\epsilon}\right\urcorner-(1 / 2) N_{\alpha, \alpha^{\prime}} \nabla_{\epsilon} \partial_{\gamma}$, and it follows that

$$
\begin{aligned}
S & =-\sum_{\alpha, \epsilon} N_{\alpha, \alpha^{\prime}}^{-1} N_{\epsilon, \epsilon^{\prime}}^{-1} \lambda_{\chi}\left(\left[X_{\epsilon^{\prime}}, Y\right]\right)\left\ulcorner\left(\nabla_{\alpha} \nabla_{\alpha^{\prime}}\right) \nabla_{\epsilon}\right\urcorner+\frac{1}{2} \sum_{\alpha, \epsilon} N_{\epsilon, \epsilon^{\prime}}^{-1} \lambda_{\chi}\left(\left[X_{\epsilon^{\prime}}, Y\right]\right) \nabla_{\epsilon} \partial_{\gamma} \\
& =\frac{\operatorname{dim}\left(V^{+}\right)}{2}\left[-\sum_{\epsilon} N_{\epsilon, \epsilon^{\prime}}^{-1} \lambda_{\chi}\left(\left[X_{\epsilon^{\prime}}, Y\right]\right)\left\ulcorner\partial_{\gamma} \nabla_{\epsilon}\right\urcorner+\sum_{\epsilon} N_{\epsilon, \epsilon^{\prime}}^{-1} \lambda_{\chi}\left(\left[X_{\epsilon^{\prime}}, Y\right]\right) \nabla_{\epsilon} \partial_{\gamma}\right],
\end{aligned}
$$

by Lemma 6.2 .

Proposition 6.2. For all $Y \in V^{-}$we have

$$
\Omega_{3}^{\prime}(Y)=\frac{1}{3} \sum_{\epsilon \in R\left(V^{+}\right)} N_{\epsilon, \epsilon^{\prime}}^{-1}\left\ulcorner\Omega_{2}\left(\left[X_{\epsilon}, Y\right]\right) \Omega_{1}\left(X_{\epsilon^{\prime}}\right)\right\urcorner .
$$

Proof. Throughout the proof, we write $Z_{\epsilon}=\left[X_{\epsilon}, Y\right]$. By Proposition 6.1 and Lemma 6.1, we have

$$
\begin{aligned}
\Omega_{3}^{\prime}(Y)=- & \frac{1}{6} \sum_{\alpha, \beta, \epsilon} N_{\beta, \beta^{\prime}}^{-1} N_{\epsilon, \epsilon^{\prime}}^{-1} M_{\beta^{\prime} \alpha}\left(\operatorname{pr}_{0}\left(Z_{\epsilon^{\prime}}\right)\right)\left\ulcorner\nabla_{\alpha} \nabla_{\beta} \nabla_{\epsilon}\right\urcorner \\
=- & \frac{1}{6} \sum_{\alpha, \beta, \epsilon} N_{\beta, \beta^{\prime}}^{-1} N_{\epsilon, \epsilon^{\prime}}^{-1} M_{\beta^{\prime} \alpha}\left(\operatorname{pr}_{0}\left(Z_{\epsilon^{\prime}}\right)\right)\left\ulcorner\left(\nabla_{\alpha} \nabla_{\beta}\right) \nabla_{\epsilon}\right\urcorner \\
& -\frac{1}{12} \sum_{\alpha, \epsilon} N_{\epsilon, \epsilon^{\prime}}^{-1} M_{\alpha \alpha}\left(\operatorname{pr}_{0}\left(Z_{\epsilon^{\prime}}\right)\right) \nabla_{\epsilon} \partial_{\gamma} .
\end{aligned}
$$

By Lemma 2.8, the sum over $\alpha$ in the second term is given by the expression $(1 / 2) \operatorname{dim}\left(V^{+}\right) \lambda_{\chi}\left(\operatorname{pr}_{0}\left(Z_{\epsilon^{\prime}}\right)\right)=0$ and so

$$
\Omega_{3}^{\prime}(Y)=-\frac{1}{6} \sum_{\alpha, \beta, \epsilon} N_{\beta, \beta^{\prime}}^{-1} N_{\epsilon, \epsilon^{\prime}}^{-1} M_{\beta^{\prime} \alpha}\left(\operatorname{pr}_{0}\left(Z_{\epsilon^{\prime}}\right)\right)\left\ulcorner\left(\nabla_{\alpha} \nabla_{\beta}\right) \nabla_{\epsilon}\right\urcorner .
$$

We observed in Section 5 that

$$
\Omega_{2}(Z)=\frac{1}{2} \sum_{\alpha, \beta} N_{\beta, \beta^{\prime}}^{-1} M_{\beta^{\prime} \alpha}(Z) \nabla_{\alpha} \nabla_{\beta}
$$


provided that $\lambda_{\chi}(Z)=0$, and it follows that

$$
\begin{aligned}
\Omega_{3}^{\prime}(Y) & =-\frac{1}{3} \sum_{\epsilon} N_{\epsilon, \epsilon^{\prime}}^{-1}\left\ulcorner\Omega_{2}\left(\operatorname{pr}_{0}\left(Z_{\epsilon^{\prime}}\right)\right) \nabla_{\epsilon}\right\urcorner \\
& =\frac{1}{3} \sum_{\epsilon} N_{\epsilon, \epsilon^{\prime}}^{-1}\left\ulcorner\Omega_{2}\left(Z_{\epsilon}\right) \Omega_{1}\left(X_{\epsilon^{\prime}}\right)\right\urcorner .
\end{aligned}
$$

Lemma 6.4. If $Z \in \operatorname{ker}\left(\lambda_{\chi}\right)$ and $W \in V^{+}$then $\left[\Omega_{2}(Z), \Omega_{1}(W)\right]=$ $\Omega_{1}([Z, W]) \partial_{\gamma}$.

Proof. It suffices to assume that $W=X_{\epsilon}$ for some $\epsilon \in R\left(V^{+}\right)$. Then

$$
\begin{aligned}
{\left[\Omega_{2}(Z), \Omega_{1}\left(X_{\epsilon}\right)\right] } & =\frac{1}{2} \sum_{\alpha, \beta} N_{\beta, \beta^{\prime}}^{-1} M_{\beta^{\prime} \alpha}(Z)\left[\nabla_{\alpha} \nabla_{\beta}, \nabla_{\epsilon}\right] \\
& =\frac{1}{2} \sum_{\alpha, \beta} N_{\beta, \beta^{\prime}}^{-1} M_{\beta^{\prime} \alpha}(Z)\left(\nabla_{\alpha}\left[\nabla_{\beta}, \nabla_{\epsilon}\right]+\left[\nabla_{\alpha}, \nabla_{\epsilon}\right] \nabla_{\beta}\right) \\
& =\sum_{\alpha, \beta} N_{\beta, \beta^{\prime}}^{-1} M_{\beta^{\prime} \alpha}(Z) \kappa_{\beta \epsilon^{\prime}} N_{\beta, \beta^{\prime}} \nabla_{\alpha} \partial_{\gamma} \\
& =\sum_{\alpha} M_{\epsilon \alpha}(Z) \Omega_{1}\left(X_{\alpha}\right) \partial_{\gamma} \\
& =\Omega_{1}\left(\left[Z, X_{\epsilon}\right]\right) \partial_{\gamma} .
\end{aligned}
$$

Lemma 6.5. For all $Y \in V^{-}$we have

$$
\sum_{\epsilon} N_{\epsilon, \epsilon^{\prime}}^{-1}\left[\left[X_{\epsilon}, Y\right], X_{\epsilon^{\prime}}\right]=-(1 / 2) \operatorname{dim}\left(V^{+}\right)\left[Y, X_{\gamma}\right] .
$$

Proof. Denote the sum on the left-hand side of the proposed identity by $S$. Then

$$
\begin{aligned}
S & =\sum_{\epsilon} N_{\epsilon, \epsilon^{\prime}}^{-1}\left[X_{\epsilon},\left[Y, X_{\epsilon^{\prime}}\right]\right]-\sum_{\epsilon} N_{\epsilon, \epsilon^{\prime}}^{-1}\left[Y,\left[X_{\epsilon}, X_{\epsilon^{\prime}}\right]\right] \\
& =-\sum_{\epsilon} N_{\epsilon, \epsilon^{\prime}}^{-1}\left[X_{\epsilon^{\prime}},\left[Y, X_{\epsilon}\right]\right]-\sum_{\epsilon}\left[Y, X_{\gamma}\right] \\
& =-\sum_{\epsilon} N_{\epsilon, \epsilon^{\prime}}^{-1}\left[\left[X_{\epsilon}, Y\right], X_{\epsilon^{\prime}}\right]-\operatorname{dim}\left(V^{+}\right)\left[Y, X_{\gamma}\right] \\
& =-S-\operatorname{dim}\left(V^{+}\right)\left[Y, X_{\gamma}\right],
\end{aligned}
$$

and the evaluation follows. 
Lemma 6.6. For all $Z \in \mathfrak{l}$ we have

$$
\sum_{\epsilon} N_{\epsilon, \epsilon^{\prime}}^{-1}\left[X_{\epsilon^{\prime}},\left[X_{\epsilon}, Z\right]\right]=(1 / 2) \operatorname{dim}\left(V^{+}\right) \lambda_{\chi}(Z) X_{\gamma}
$$

Proof. Denote the sum on the left-hand side of the proposed identity by $S$. Then

$$
\begin{aligned}
S & =\sum_{\epsilon} N_{\epsilon, \epsilon^{\prime}}^{-1}\left[\left[X_{\epsilon^{\prime}}, X_{\epsilon}\right], Z\right]+\sum_{\epsilon} N_{\epsilon, \epsilon^{\prime}}^{-1}\left[X_{\epsilon},\left[X_{\epsilon^{\prime}}, Z\right]\right] \\
& =\sum_{\epsilon}\left[Z, X_{\gamma}\right]-\sum_{\epsilon} N_{\epsilon, \epsilon^{\prime}}^{-1}\left[X_{\epsilon^{\prime}},\left[X_{\epsilon}, Z\right]\right] \\
& =\operatorname{dim}\left(V^{+}\right) \lambda_{\chi}(Z) X_{\gamma}-S
\end{aligned}
$$

and the evaluation follows.

Lemma 6.7. For all $W \in V^{+}$we have

$$
W=\sum_{\epsilon \in R\left(V^{+}\right)} N_{\epsilon, \epsilon^{\prime}}^{-1}\left\langle W, X_{\epsilon^{\prime}}\right\rangle X_{\epsilon} .
$$

Proof. This follows at once from the non-degeneracy of $\langle\cdot, \cdot\rangle$.

Proposition 6.3. For all $Y \in V^{-}$we have

$$
\begin{aligned}
& \Omega_{3}^{\prime}(Y)= \\
& \frac{1}{3} \sum_{\epsilon \in R\left(V^{+}\right)} N_{\epsilon, \epsilon^{\prime}}^{-1} \Omega_{1}\left(X_{\epsilon^{\prime}}\right) \Omega_{2}\left(\left[X_{\epsilon}, Y\right]\right)-(1 / 12)\left(\operatorname{dim}\left(V^{+}\right)+1\right) \Omega_{1}\left(\left[Y, X_{\gamma}\right]\right) \partial_{\gamma} .
\end{aligned}
$$

Proof. Let $Z_{\epsilon}=\left[X_{\epsilon}, Y\right]$. With this notation, Proposition 6.2 may be rewritten as

$$
\Omega_{3}^{\prime}(Y)=\frac{1}{3} \sum_{\epsilon} N_{\epsilon, \epsilon^{\prime}}^{-1} \Omega_{1}\left(X_{\epsilon^{\prime}}\right) \Omega_{2}\left(Z_{\epsilon}\right)+\frac{1}{6} \sum_{\epsilon} N_{\epsilon, \epsilon^{\prime}}^{-1}\left[\Omega_{2}\left(Z_{\epsilon}\right), \Omega_{1}\left(X_{\epsilon^{\prime}}\right)\right] .
$$

All that remains is to evaluate the second term. By Lemma 6.4,

$$
\begin{aligned}
{\left[\Omega_{2}\left(Z_{\epsilon}\right), \Omega_{1}\left(X_{\epsilon^{\prime}}\right)\right] } & =\left[\Omega_{2}\left(Z_{\epsilon}-(1 / 2) \lambda_{\chi}\left(Z_{\epsilon}\right) H_{\gamma}\right), \Omega_{1}\left(X_{\epsilon^{\prime}}\right)\right] \\
& =\Omega_{1}\left(\left[Z_{\epsilon}-(1 / 2) \lambda_{\chi}\left(Z_{\epsilon}\right) H_{\gamma}, X_{\epsilon^{\prime}}\right]\right) \partial_{\gamma} \\
& =\Omega_{1}\left(\left[Z_{\epsilon}, X_{\epsilon^{\prime}}\right]\right) \partial_{\gamma}-(1 / 2) \lambda_{\chi}\left(Z_{\epsilon}\right) \Omega_{1}\left(X_{\epsilon^{\prime}}\right) \partial_{\gamma} .
\end{aligned}
$$

Lemma 6.5 gives

$$
\sum_{\epsilon} N_{\epsilon, \epsilon^{\prime}}^{-1} \Omega_{1}\left(\left[Z_{\epsilon}, X_{\epsilon^{\prime}}\right]\right) \partial_{\gamma}=-(1 / 2) \operatorname{dim}\left(V^{+}\right) \Omega_{1}\left(\left[Y, X_{\gamma}\right]\right) \partial_{\gamma}
$$


and, by Lemma 6.3,

$$
\begin{aligned}
\sum_{\epsilon} N_{\epsilon, \epsilon^{\prime}}^{-1} \lambda_{\chi}\left(Z_{\epsilon}\right) \Omega_{1}\left(X_{\epsilon^{\prime}}\right) \partial_{\gamma} & =\Omega_{1}\left(\sum_{\epsilon} N_{\epsilon, \epsilon^{\prime}}^{-1}\left\langle X_{\epsilon},\left[Y, X_{\gamma}\right]\right\rangle X_{\epsilon^{\prime}}\right) \partial_{\gamma} \\
& =\Omega_{1}\left(\sum_{\epsilon} N_{\epsilon, \epsilon^{\prime}}^{-1}\left\langle\left[Y, X_{\gamma}\right], X_{\epsilon^{\prime}}\right\rangle X_{\epsilon}\right) \partial_{\gamma} \\
& =\Omega_{1}\left(\left[Y, X_{\gamma}\right]\right) \partial_{\gamma},
\end{aligned}
$$

where we have used Lemma 6.7 at the last step.

For $Y \in V^{-}$, let us define

$$
\tilde{\Omega}_{3}(Y)=\sum_{\epsilon \in R(V+)} N_{\epsilon, \epsilon^{\prime}}^{-1} \Omega_{1}\left(X_{\epsilon^{\prime}}\right) \Omega_{2}\left(\left[X_{\epsilon}, Y\right]\right)
$$

and

$$
C_{3}(Y)=\Omega_{1}\left(\left[Y, X_{\gamma}\right]\right) \partial_{\gamma},
$$

so that the result of Proposition 6.3 may be written as

$$
\Omega_{3}^{\prime}(Y)=(1 / 3) \tilde{\Omega}_{3}(Y)-(1 / 12)\left(\operatorname{dim}\left(V^{+}\right)+1\right) C_{3}(Y) .
$$

A routine computation gives $C_{3}(\operatorname{Ad}(l) Y)=\chi(l)^{-2} l \cdot C_{3}(Y)$ for all $l \in L$ and $Y \in V^{-}$; that is, $C_{3}$ enjoys the same transformation law as $\Omega_{3}$. It follows that $\tilde{\Omega}_{3}$ also has the same transformation law. As usual, we extend $\tilde{\Omega}_{3}$ and $C_{3}$ to be left $\mathbb{C}[\mathfrak{n}]$-linear.

We shall evaluate the commutators of $\tilde{\Omega}_{3}(Y)$ and $C_{3}(Y)$ with $D_{-\gamma}$ separately. It will emerge that it is not $\Omega_{3}^{\prime}$ itself, but rather a different linear combination of $\tilde{\Omega}_{3}$ and $C_{3}$, that will yield a conformally invariant system for a suitable value of $s$. Because $\tilde{\Omega}_{3}$ is substantially more complicated than $C_{3}$, we regard the multiple of $C_{3}$ that must be added to $\Omega_{3}^{\prime}$ to obtain conformal invariance as a "correction term"; whence the notation chosen for it. It will emerge that there is a unique line in the plane spanned by $\tilde{\Omega}_{3}$ and $C_{3}$ on which conformal invariance holds (for a suitable value of $s$ ). In this sense at least, the construction is canonical.

In what follows, the notational conventions are those already employed in Section 5. In particular, we shall write $X=\sum_{\alpha \in R\left(V^{+}\right)} \xi_{\alpha} X_{\alpha}$ for the generic element of $V^{+}$.

Lemma 6.8. For all $W \in V^{+}$we have $\left[\Omega_{1}(W), \xi_{\gamma}\right]=-(1 / 2)\langle W, X\rangle$.

Proof. It suffices to verify this for $W=X_{\alpha}$. In this case, $\Omega_{1}(W)=\nabla_{\alpha}$ and the claim follows directly from the definition of $\nabla_{\alpha}$. 
Lemma 6.9. For all $Y \in V^{-}$we have

$$
\left[\Omega_{1}\left(\left[Y, X_{\gamma}\right]\right), \Omega_{1}(X)\right]=\lambda_{\chi}([Y, X]) \partial_{\gamma}+\Omega_{1}\left(\left[Y, X_{\gamma}\right]\right) .
$$

Proof. Well,

$$
\begin{aligned}
& {\left[\Omega_{1}\left(\left[Y, X_{\gamma}\right]\right), \Omega_{1}(X)\right]} \\
& =\sum_{\mu}\left[\Omega_{1}\left(\left[Y, X_{\gamma}\right]\right), \xi_{\mu} \Omega_{1}\left(X_{\mu}\right)\right] \\
& =\sum_{\mu} \xi_{\mu}\left[\Omega_{1}\left(\left[Y, X_{\gamma}\right]\right), \Omega_{1}\left(X_{\mu}\right)\right]+\sum_{\mu}\left[\Omega_{1}\left(\left[Y, X_{\gamma}\right]\right), \xi_{\mu}\right] \Omega_{1}\left(X_{\mu}\right) \\
& =\sum_{\mu} \xi_{\mu} \Omega_{1}\left(\left[\left[Y, X_{\gamma}\right], X_{\mu}\right]\right)+\sum_{\mu} \xi_{\mu}\left(\left[Y, X_{\gamma}\right]\right) \Omega_{1}\left(X_{\mu}\right) \\
& =\Omega_{1}\left(\left[\left[Y, X_{\gamma}\right], X\right]\right)+\Omega_{1}\left(\left[Y, X_{\gamma}\right]\right) \\
& =\Omega_{1}\left(\left[[Y, X], X_{\gamma}\right]\right)+\Omega_{1}\left(\left[Y, X_{\gamma}\right]\right) \\
& =\lambda_{\chi}([Y, X]) \partial_{\gamma}+\Omega_{1}\left(\left[Y, X_{\gamma}\right]\right) .
\end{aligned}
$$

In the fourth line, $\xi_{\mu}\left(\left[Y, X_{\gamma}\right]\right)$ represents evaluation of the functional $\xi_{\mu}$ on the vector $\left[Y, X_{\gamma}\right]$; we mention this because the notation has not previously occurred.

Proposition 6.4. For all $Y \in V^{-}$we have

$$
\begin{array}{r}
{\left[D_{-\gamma}, C_{3}(Y)\right]=-3 \xi_{\gamma} C_{3}(Y)+C_{3}\left(\left[\tau_{2}(X), Y\right]\right)+s \lambda_{\chi}([Y, X]) \partial_{\gamma}} \\
-(2 s+1) \Omega_{1}\left(\left[Y, X_{\gamma}\right]\right)-\Omega_{1}(X) \Omega_{1}\left(\left[Y, X_{\gamma}\right]\right) .
\end{array}
$$

Proof. Recall from Proposition 4.2 that

$$
D_{-\gamma}=-P \partial_{\gamma}+\xi_{\gamma} E+\{\Delta, \cdot\}+2 s \xi_{\gamma},
$$

where $E=2 \xi_{\gamma} \partial_{\gamma}+\sum_{\alpha} \xi_{\alpha} \partial_{\alpha}$. A computation using this formula gives that $\left[D_{-\gamma}, \partial_{\gamma}\right]=-(E+2 s)$. By substituting the identity

$$
\partial_{\alpha}=\Omega_{1}\left(X_{\alpha}\right)+(1 / 2) N_{\alpha, \alpha^{\prime}} \xi_{\alpha^{\prime}} \partial_{\gamma}
$$

into the definition of $E$ and simplifying, we obtain $E=\Omega_{1}(X)+2 \xi_{\gamma} \partial_{\gamma}$. Now

$$
\left[D_{-\gamma}, C_{3}(Y)\right]=\left[D_{-\gamma}, \Omega_{1}\left(\left[Y, X_{\gamma}\right]\right)\right] \partial_{\gamma}+\Omega_{1}\left(\left[Y, X_{\gamma}\right]\right)\left[D_{-\gamma}, \partial_{\gamma}\right]
$$


and, using the above observations and Lemmas 6.8 and 6.9,

$$
\begin{aligned}
\Omega_{1}\left(\left[Y, X_{\gamma}\right]\right)\left[D_{-\gamma}, \partial_{\gamma}\right]= & -\Omega_{1}\left(\left[Y, X_{\gamma}\right]\right)\left(2 \xi_{\gamma} \partial_{\gamma}+\Omega_{1}(X)+2 s\right) \\
= & -2 \xi_{\gamma} \Omega_{1}\left(\left[Y, X_{\gamma}\right]\right) \partial_{\gamma}-2\left[\Omega_{1}\left(\left[Y, X_{\gamma}\right]\right), \xi_{\gamma}\right] \partial_{\gamma} \\
& -2 s \Omega_{1}\left(\left[Y, X_{\gamma}\right]\right)-\Omega_{1}\left(\left[Y, X_{\gamma}\right]\right) \Omega_{1}(X) \\
= & -2 \xi_{\gamma} C_{3}(Y)+\left\langle\left[Y, X_{\gamma}\right], X\right\rangle \partial_{\gamma}-2 s \Omega_{1}\left(\left[Y, X_{\gamma}\right]\right) \\
& -\Omega_{1}(X) \Omega_{1}\left(\left[Y, X_{\gamma}\right]\right)-\lambda_{\chi}([Y, X]) \partial_{\gamma}-\Omega_{1}\left(\left[Y, X_{\gamma}\right]\right) \\
= & -2 \xi_{\gamma} C_{3}(Y)+\lambda_{\chi}([Y, X]) \partial_{\gamma}-2 s \Omega_{1}\left(\left[Y, X_{\gamma}\right]\right) \\
& -\Omega_{1}(X) \Omega_{1}([Y, X,])-\lambda_{\chi}([Y, X]) \partial_{\gamma}-\Omega_{1}\left(\left[Y, X_{\gamma}\right]\right) \\
= & -2 \xi_{\gamma} C_{3}(Y)-(2 s+1) \Omega_{1}\left(\left[Y, X_{\gamma}\right]\right)-\Omega_{1}(X) \Omega_{1}\left(\left[Y, X_{\gamma}\right]\right) .
\end{aligned}
$$

By Theorem 5.1,

$$
\begin{aligned}
{\left[D_{-\gamma}, \Omega_{1}\left(\left[Y, X_{\gamma}\right]\right)\right] } & =-\xi_{\gamma} \Omega_{1}\left(\left[Y, X_{\gamma}\right]\right)+\Omega_{1}\left(\left[\tau_{2}(X),\left[Y, X_{\gamma}\right]\right]\right)+s\left\langle\left[Y, X_{\gamma}\right], X\right\rangle \\
& =-\xi_{\gamma} \Omega_{1}\left(\left[Y, X_{\gamma}\right]\right)+\Omega_{1}\left(\left[\left[\tau_{2}(X), Y\right], X_{\gamma}\right]\right)+s \lambda_{\chi}([Y, X]),
\end{aligned}
$$

because $\left[\tau_{2}(X), X_{\gamma}\right]=0$. Thus

$$
\left[D_{-\gamma}, \Omega_{1}\left(\left[Y, X_{\gamma}\right]\right)\right] \partial_{\gamma}=-\xi_{\gamma} C_{3}(Y)+C_{3}\left(\left[\tau_{2}(X), Y\right]\right)+s \lambda_{\chi}([Y, X]) \partial_{\gamma} .
$$

Adding these evaluations completes the proof.

Lemma 6.10. If $Z \in \mathfrak{l}$ and $\lambda_{\chi}(Z)=0$ then

$$
\left[\Omega_{2}(Z), \xi_{\gamma}\right]=-(1 / 2) \Omega_{1}([Z, X])
$$

and

$$
\left[\Omega_{2}(Z), \xi_{\alpha}\right]=N_{\alpha, \alpha^{\prime}}^{-1} \Omega_{1}\left(\left[Z, X_{\alpha^{\prime}}\right]\right)
$$

Proof. We have

$$
\left[\Omega_{2}(Z), \xi_{\gamma}\right]=\frac{1}{2} \sum_{\beta} N_{\beta, \beta^{\prime}}^{-1}\left[\Omega_{1}\left(X_{\beta}\right) \Omega_{1}\left(\left[Z, X_{\beta^{\prime}}\right]\right), \xi_{\gamma}\right]
$$

and $\left[\Omega_{1}\left(X_{\alpha}\right), \xi_{\gamma}\right]=-(1 / 2) N_{\alpha, \alpha^{\prime}} \xi_{\alpha^{\prime}}$. From this point, a routine computation completes the proof. The second identity is similar.

Lemma 6.11. For all $Z \in \mathfrak{l}$ we have

$$
\sum_{\epsilon} N_{\epsilon, \epsilon^{\prime}}^{-1} \Omega_{1}\left(\left[Z, X_{\epsilon}\right]\right) \Omega_{1}\left(X_{\epsilon^{\prime}}\right)=-2 \Omega_{2}(Z)+(1 / 4) \lambda_{\chi}(Z) \operatorname{dim}\left(V^{+}\right) \partial_{\gamma} .
$$


Proof. Since $Z=\operatorname{pr}_{0}(Z)+(1 / 2) \lambda_{\chi}(Z) H_{\gamma}$, the left-hand side is

$$
\sum_{\epsilon} N_{\epsilon, \epsilon^{\prime}}^{-1} \Omega_{1}\left(\left[\operatorname{pr}_{0}(Z), X_{\epsilon}\right]\right) \Omega_{1}\left(X_{\epsilon^{\prime}}\right)+\frac{1}{2} \lambda_{\chi}(Z) \sum_{\epsilon} N_{\epsilon, \epsilon^{\prime}}^{-1} \Omega_{1}\left(X_{\epsilon}\right) \Omega_{1}\left(X_{\epsilon^{\prime}}\right) .
$$

We observed in Section 5 that the first term is equal to $-2 \Omega_{2}\left(\operatorname{pr}_{0}(Z)\right)=$ $-2 \Omega_{2}(Z)$. By Lemma 6.2, the second term is $(1 / 2) \lambda_{\chi}(Z) \cdot(1 / 2) \operatorname{dim}\left(V^{+}\right) \partial_{\gamma}$.

Recall the constants $c(\mathfrak{g}, \mathcal{C})$ and $p(\mathfrak{g}, \mathcal{C})$ that were introduced in Section 2 for each algebra $\mathfrak{g}$ and each component $\mathcal{C}$ of $\mathcal{D}_{\gamma}(\mathfrak{g}, \mathfrak{h})$. When $\mathcal{D}_{\gamma}(\mathfrak{g}, \mathfrak{h})$ has a single component, we abbreviate the names of these constants to $c(\mathfrak{g})$ and $p(\mathfrak{g})$. Note that, amongst the algebras considered in this section, $\mathcal{D}_{\gamma}(\mathfrak{g}, \mathfrak{h})$ has a single component for the exceptional algebras and for the algebras of type $\mathrm{A}_{r}$.

Theorem 6.1. Suppose that $\mathfrak{g}$ is an exceptional algebra and define

$$
\Omega_{3}(Y)=(1 / 3) \tilde{\Omega}_{3}(Y)+(1 / 12)(c(\mathfrak{g})-p(\mathfrak{g})) C_{3}(Y) .
$$

For all $Y \in V^{-}$we have

$$
\begin{aligned}
{\left[D_{-\gamma}, \Omega_{3}(Y)\right]=- } & 3 \xi_{\gamma} \Omega_{3}(Y)+\Omega_{3}\left(\left[\tau_{2}(X), Y\right]\right) \\
+ & \left(s-s_{3}\right)\left(\Omega_{2}([Y, X])+(1 / 4)(c(\mathfrak{g})-2) \lambda_{\chi}([Y, X]) \partial_{\gamma}\right. \\
& \left.-(1 / 2)(c(\mathfrak{g})-1) \Omega_{1}\left(\left[Y, X_{\gamma}\right]\right)-(1 / 2) \Omega_{1}(X) \Omega_{1}\left(\left[Y, X_{\gamma}\right]\right)\right),
\end{aligned}
$$

where $s_{3}=\left(\operatorname{dim}\left(V^{+}\right)-2\right) / 6$.

Proof. We begin by computing the commutator of $\tilde{\Omega}_{3}(Y)$ and $D_{-\gamma}$. As usual, we shall express this commutator as the sum of a number of terms, and evaluate each separately. Only some of these evaluations will require the assumption that $\mathfrak{g}$ is an exceptional algebra, and we shall point out this dependence as we go. We shall obtain the identity

$$
\begin{aligned}
{\left[D_{-\gamma}, \tilde{\Omega}_{3}(Y)\right]=} & -3 \xi_{\gamma} \tilde{\Omega}_{3}(Y)+\tilde{\Omega}_{3}\left(\left[\tau_{2}(X), Y\right]\right)+3\left(s-s_{3}\right) \Omega_{2}([Y, X]) \\
+ & \left(s-s_{2}\right)\left((1 / 4)(d-2) \lambda_{\chi}([Y, X]) \partial_{\gamma}-(1 / 2)(d+1) \Omega_{1}\left(\left[Y, X_{\gamma}\right]\right)\right. \\
& \left.-(3 / 2) \Omega_{1}(X) \Omega_{1}\left(\left[Y, X_{\gamma}\right]\right)\right)
\end{aligned}
$$

where $d=\operatorname{dim}\left(V^{+}\right), s_{3}$ is as in the statement, and $s_{2}=(1 / 2) c(\mathfrak{g})-1$, as in Theorem 5.2.

For all $l \in L$ we have

$$
\left[D_{-\gamma}, \tilde{\Omega}_{3}(\operatorname{Ad}(l) Y)\right]=\chi(l)^{-1} l \cdot\left[D_{-\gamma}, \tilde{\Omega}_{3}(Y)\right]
$$


and the right-hand side of the proposed identity satisfies the same transformation law under $L$. Now the prehomogeneous vector space $\left(L, \mathrm{Ad}, V^{-}\right)$is equivalent to $\left(L, \mathrm{Ad}, V^{+}\right)$via the map $\tau_{1}^{-1}$. By Proposition 3.3, $X_{0}=\sqrt{2}\left(X_{\delta}+X_{\delta^{\prime}}\right)$ is a generic point of $\left(L, \mathrm{Ad}, V^{+}\right)$, and it follows that $\tau_{1}\left(X_{0}\right)=\sqrt{2}\left(X_{-\delta}-X_{-\delta^{\prime}}\right)$ is a generic point of $\left(L, \mathrm{Ad}, V^{-}\right)$. Thus it suffices to establish the identity for $Y=\tau_{1}\left(X_{0}\right)$. But the identity is linear in $Y$, and so it suffices to prove it for $Y=X_{-\delta}$ and $Y=X_{-\delta^{\prime}}$ separately. We could, to begin with, have chosen $\delta^{\prime}$ to play the role of $\delta$, and so the identity for $Y=X_{-\delta^{\prime}}$ follows from the identity for $Y=X_{-\delta}$. Thus we are reduced to proving the identity for $Y=X_{-\delta}$. We shall assume this equality henceforth, and use $Y$ and $X_{-\delta}$ interchangeably. As in the proof of Theorem 5.2, the majority of the calculations are valid for any $Y$, and it would be distracting to replace $Y$ by $X_{-\delta}$ in these.

For $\epsilon \in R\left(V^{+}\right)$, we write $Z_{\epsilon}=\left[X_{\epsilon}, Y\right]$. From the definition, we have

$$
\begin{aligned}
{\left[D_{-\gamma}, \tilde{\Omega}_{3}(Y)\right]=} & \sum_{\epsilon} N_{\epsilon, \epsilon^{\prime}}^{-1}\left[D_{-\gamma}, \Omega_{1}\left(X_{\epsilon^{\prime}}\right)\right] \Omega_{2}\left(Z_{\epsilon}\right) \\
& +\sum_{\epsilon} N_{\epsilon, \epsilon^{\prime}}^{-1} \Omega_{1}\left(X_{\epsilon^{\prime}}\right)\left[D_{-\gamma}, \Omega_{2}\left(Z_{\epsilon}\right)\right] .
\end{aligned}
$$

Both $\left[D_{-\gamma}, \Omega_{1}\left(X_{\epsilon^{\prime}}\right)\right]$ and $\left[D_{-\gamma}, \Omega_{2}\left(Z_{\epsilon}\right)\right]$ may be expressed as a sum of three terms. We consider the contribution to (6.1) from these terms in turn. The contribution from the first terms is

$$
\begin{aligned}
T_{1} & =\sum_{\epsilon} N_{\epsilon, \epsilon^{\prime}}^{-1}\left(-\xi_{\gamma} \Omega_{1}\left(X_{\epsilon^{\prime}}\right)\right) \Omega_{2}\left(Z_{\epsilon}\right)+\sum_{\epsilon} N_{\epsilon, \epsilon^{\prime}}^{-1} \Omega_{1}\left(X_{\epsilon^{\prime}}\right)\left(-2 \xi_{\gamma} \Omega_{2}\left(Z_{\epsilon}\right)\right) \\
& =-3 \xi_{\gamma} \sum_{\epsilon} N_{\epsilon, \epsilon^{\prime}}^{-1} \Omega_{1}\left(X_{\epsilon^{\prime}}\right) \Omega_{2}\left(Z_{\epsilon}\right)-2 \sum_{\epsilon} N_{\epsilon, \epsilon^{\prime}}^{-1}\left[\Omega_{1}\left(X_{\epsilon^{\prime}}\right), \xi_{\gamma}\right] \Omega_{2}\left(Z_{\epsilon}\right) \\
& =-3 \xi_{\gamma} \tilde{\Omega}_{3}(Y)+\sum_{\epsilon} N_{\epsilon, \epsilon^{\prime}}^{-1}\left\langle X_{\epsilon^{\prime}}, X\right\rangle \Omega_{2}\left(Z_{\epsilon}\right) \\
& =-3 \xi_{\gamma} \tilde{\Omega}_{3}(Y)+\sum_{\epsilon} N_{\epsilon, \epsilon^{\prime}}^{-1} N_{\epsilon^{\prime}, \epsilon} \xi_{\epsilon} \Omega_{2}\left(\left[X_{\epsilon}, Y\right]\right) \\
& =-3 \xi_{\gamma} \tilde{\Omega}_{3}(Y)-\Omega_{2}([X, Y]) \\
& =-3 \xi_{\gamma} \tilde{\Omega}_{3}(Y)+\Omega_{2}([Y, X]) .
\end{aligned}
$$

Note that this evaluation is independent of any assumption on the type of $\mathfrak{g}$.

We now consider the contribution from the second terms. It is

$$
T_{2}=\sum_{\epsilon} N_{\epsilon, \epsilon^{\prime}}^{-1} \Omega_{1}\left(\left[\tau_{2}(X), X_{\epsilon^{\prime}}\right]\right) \Omega_{2}\left(Z_{\epsilon}\right)+\sum_{\epsilon} N_{\epsilon, \epsilon^{\prime}}^{-1} \Omega_{1}\left(X_{\epsilon^{\prime}}\right) \Omega_{2}\left(\left[\tau_{2}(X), Z_{\epsilon}\right]\right) .
$$

Note that we do not have to concern ourselves with the difference between $Z_{\epsilon}$ and $\operatorname{pr}_{0}\left(Z_{\epsilon}\right)$ here, because $Z_{\epsilon}-\operatorname{pr}_{0}\left(Z_{\epsilon}\right)$ lies in the center of $\mathfrak{l}$ and, in particular, 
commutes with $\tau_{2}(X)$. Throughout the proof, we shall use a hierarchical numbering scheme for terms. For example, the first term in the previous identity will be called $T_{21}$ and the second term $T_{22}$. With this notation,

$$
\begin{aligned}
& T_{22}=\sum_{\epsilon} N_{\epsilon, \epsilon^{\prime}}^{-1} \Omega_{1}\left(X_{\epsilon^{\prime}}\right) \Omega_{2}\left(\left[\tau_{2}(X),\left[X_{\epsilon}, Y\right]\right]\right) \\
& =\sum_{\epsilon} N_{\epsilon, \epsilon^{\prime}}^{-1} \Omega_{1}\left(X_{\epsilon^{\prime}}\right)\left(\Omega_{2}\left(\left[\left[\tau_{2}(X), X_{\epsilon}\right], Y\right]\right)+\Omega_{2}\left(\left[X_{\epsilon},\left[\tau_{2}(X), Y\right]\right]\right)\right) \\
& =\sum_{\epsilon, \mu} N_{\epsilon, \epsilon^{\prime}}^{-1} \Omega_{1}\left(X_{\epsilon^{\prime}}\right)\left(M_{\epsilon \mu}\left(\tau_{2}(X)\right) \Omega_{2}\left(\left[X_{\mu}, Y\right]\right)+M_{\delta \mu}^{-}\left(\tau_{2}(X)\right) \Omega_{2}\left(\left[X_{\epsilon}, X_{-\mu}\right]\right)\right) \\
& =\sum_{\epsilon, \mu} N_{\epsilon, \epsilon^{\prime}}^{-1} M_{\epsilon \mu}\left(\tau_{2}(X)\right) \Omega_{1}\left(X_{\epsilon^{\prime}}\right) \Omega_{2}\left(\left[X_{\mu}, Y\right]\right) \\
& +\sum_{\epsilon, \mu} N_{\epsilon, \epsilon^{\prime}}^{-1}\left[\Omega_{1}\left(X_{\epsilon^{\prime}}\right), M_{\epsilon \mu}\left(\tau_{2}(X)\right)\right] \Omega_{2}\left(\left[X_{\mu}, Y\right]\right) \\
& +\sum_{\epsilon, \mu} N_{\epsilon, \epsilon^{\prime}}^{-1} M_{\delta \mu}^{-}\left(\tau_{2}(X)\right) \Omega_{1}\left(X_{\epsilon^{\prime}}\right) \Omega_{2}\left(\left[X_{\epsilon}, X_{-\mu}\right]\right) \\
& +\sum_{\epsilon, \mu} N_{\epsilon, \epsilon^{\prime}}^{-1}\left[\Omega_{1}\left(X_{\epsilon^{\prime}}\right), M_{\delta \mu}^{-}\left(\tau_{2}(X)\right)\right] \Omega_{2}\left(\left[X_{\epsilon}, X_{-\mu}\right]\right) \\
& =-\sum_{\epsilon, \mu} N_{\epsilon, \epsilon^{\prime}}^{-1} M_{\epsilon^{\prime} \mu}\left(\tau_{2}(X)\right) \Omega_{1}\left(X_{\epsilon}\right) \Omega_{2}\left(\left[X_{\mu}, Y\right]\right) \\
& +\sum_{\epsilon, \mu} N_{\epsilon, \epsilon^{\prime}}^{-1} M_{\epsilon \mu}\left(\partial_{\epsilon^{\bullet}} \bullet \tau_{2}(X)\right) \Omega_{2}\left(\left[X_{\mu}, Y\right]\right)+\sum_{\mu} M_{\delta \mu}^{-}\left(\tau_{2}(X)\right) \tilde{\Omega}_{3}\left(X_{-\mu}\right) \\
& +\sum_{\epsilon, \mu} N_{\epsilon, \epsilon^{\prime}}^{-1} M_{\delta \mu}^{-}\left(\partial_{\epsilon^{\prime}} \bullet \tau_{2}(X)\right) \Omega_{2}\left(\left[X_{\epsilon}, X_{-\mu}\right]\right) \\
& =-\sum_{\epsilon, \mu} N_{\mu, \mu^{\prime}}^{-1} M_{\mu^{\prime} \epsilon}\left(\tau_{2}(X)\right) \Omega_{1}\left(X_{\epsilon}\right) \Omega_{2}\left(\left[X_{\mu}, Y\right]\right) \\
& +\sum_{\epsilon} N_{\epsilon, \epsilon^{\prime}}^{-1} \Omega_{2}\left(\left[\left[\partial_{\epsilon^{\bullet}} \bullet \tau_{2}(X), X_{\epsilon}\right], Y\right]\right)+\tilde{\Omega}_{3}\left(\left[\tau_{2}(X), Y\right]\right) \\
& +\sum_{\epsilon} N_{\epsilon, \epsilon^{\prime}}^{-1} \Omega_{2}\left(\left[X_{\epsilon},\left[\partial_{\epsilon^{\prime}} \bullet \tau_{2}(X), Y\right]\right]\right) \\
& =-\sum_{\mu} N_{\mu, \mu^{\prime}}^{-1} \Omega_{1}\left(\left[\tau_{2}(X), X_{\mu^{\prime}}\right]\right) \Omega_{2}\left(\left[X_{\mu}, Y\right]\right)+\tilde{\Omega}_{3}\left(\left[\tau_{2}(X), Y\right]\right) \\
& +\sum_{\epsilon} N_{\epsilon, \epsilon^{\prime}}^{-1} \Omega_{2}\left(\left[\partial_{\epsilon^{\prime}} \bullet \tau_{2}(X),\left[X_{\epsilon}, Y\right]\right]\right) .
\end{aligned}
$$

Note that the first term here is the negative of $T_{21}$, and so we arrive at

$$
T_{2}=\tilde{\Omega}_{3}\left(\left[\tau_{2}(X), Y\right]\right)+\sum_{\epsilon} N_{\epsilon, \epsilon^{\prime}}^{-1} \Omega_{2}\left(\left[\partial_{\epsilon^{\prime}} \bullet \tau_{2}(X),\left[X_{\epsilon}, Y\right]\right]\right) .
$$

By Lemma 3.6, $\partial_{\epsilon^{\prime}} \bullet \tau_{2}(X)=(1 / 2)\left\langle X_{\epsilon^{\prime}}, X\right\rangle H_{\gamma}+N_{\epsilon^{\prime},-\gamma}\left[X, X_{-\epsilon}\right]$. Note that 
$\left[X_{\epsilon}, Y\right] \in \mathfrak{l}$ and so $\left[H_{\gamma},\left[X_{\epsilon}, Y\right]\right]=0$. From these observations, we obtain

$$
T_{2}=\tilde{\Omega}_{3}\left(\left[\tau_{2}(X), Y\right]\right)+\sum_{\epsilon} N_{\epsilon, \epsilon^{\prime}}^{-1} N_{\epsilon^{\prime},-\gamma} \Omega_{2}\left(\left[\left[X, X_{-\epsilon}\right],\left[X_{\epsilon}, Y\right]\right]\right) .
$$

We have seen that $N_{\epsilon^{\prime},-\gamma}=-(1 / 2)\|\epsilon\|^{2} N_{\epsilon^{\prime}, \epsilon}$ and so $N_{\epsilon^{\prime},-\gamma} N_{\epsilon, \epsilon^{\prime}}^{-1}=(1 / 2)\|\epsilon\|^{2}$. Therefore

$$
\begin{aligned}
T_{2} & \left.=\tilde{\Omega}_{3}\left(\left[\tau_{2}(X), Y\right]\right)+\frac{1}{2} \Omega_{2}\left(\sum_{\epsilon}\|\epsilon\|^{2}\left[\left[X, X_{-\epsilon}\right],[X, Y Y]\right]\right)\right) \\
& =\tilde{\Omega}_{3}\left(\left[\tau_{2}(X), Y\right]\right)-\frac{1}{2} \sum_{\mathcal{C}} p(\mathfrak{g}, \mathcal{C}) \Omega_{2}\left(\operatorname{pr}_{\mathcal{C}}([Y, X])\right),
\end{aligned}
$$

by Proposition 2.2. Recall that the sum on the right-hand side is over all components $\mathcal{C}$ of $\mathcal{D}_{\gamma}(\mathfrak{g}, \mathfrak{h})$. Expressed in this form, the evaluation does not depend on any assumption about the type of $\mathfrak{g}$. When $\mathfrak{g}$ is exceptional, it may be simplified to

$$
T_{2}=\tilde{\Omega}_{3}\left(\left[\tau_{2}(X), Y\right]\right)-(1 / 2) p(\mathfrak{g}) \Omega_{2}([Y, X]) .
$$

It remains to consider the contribution to (6.1) made by the third terms in the formulas for $\left[D_{-\gamma}, \Omega_{1}\left(X_{\epsilon^{\prime}}\right)\right]$ and $\left[D_{-\gamma}, \Omega_{2}\left(Z_{\epsilon}\right)\right]$. We shall evaluate these separately. For the former, we obtain

$$
\begin{aligned}
T_{3} & =s \sum_{\epsilon} N_{\epsilon, \epsilon^{\prime}}^{-1}\left\langle X_{\epsilon^{\prime}}, X\right\rangle \Omega_{2}\left(\left[X_{\epsilon}, Y\right]\right) \\
& =-s \sum_{\epsilon} \xi_{\epsilon} \Omega_{2}\left(\left[X_{\epsilon}, Y\right]\right) \\
& =s \Omega_{2}([Y, X]) .
\end{aligned}
$$

Again, this evaluation is independent of the type of $\mathfrak{g}$.

The remainder of the evaluation does rely on the assumption that $\mathfrak{g}$ is exceptional, which implies that $\mathcal{D}_{\gamma}(\mathfrak{g}, \mathfrak{h})$ has a single component, $\mathcal{C}$, and that $\operatorname{pr}_{0}\left(Z_{\epsilon}\right) \in \mathfrak{l}(\mathcal{C})$. The contribution to (6.1) from the remaining term is thus $\left(s-s_{2}\right)$ times

$$
\begin{aligned}
T_{4} & =\sum_{\epsilon} N_{\epsilon, \epsilon^{\prime}}^{-1} \Omega_{1}\left(X_{\epsilon^{\prime}}\right) \Omega_{1}\left(\left[\operatorname{pr}_{0}\left(Z_{\epsilon}\right), X\right]\right) \\
& =\sum_{\epsilon} N_{\epsilon, \epsilon^{\prime}}^{-1} \Omega_{1}\left(\left[\operatorname{pr}_{0}\left(Z_{\epsilon}\right), X\right]\right) \Omega_{1}\left(X_{\epsilon^{\prime}}\right)+\sum_{\epsilon} N_{\epsilon, \epsilon^{\prime}}^{-1}\left[\Omega_{1}\left(X_{\epsilon^{\prime}}\right), \Omega_{1}\left(\left[\operatorname{pr}_{0}\left(Z_{\epsilon}\right), X\right]\right)\right] .
\end{aligned}
$$

Now

$$
T_{41}=\sum_{\epsilon} N_{\epsilon, \epsilon^{\prime}}^{-1} \Omega_{1}\left(\left[\operatorname{pr}_{0}\left(Z_{\epsilon}\right), X\right]\right) \Omega_{1}\left(X_{\epsilon^{\prime}}\right)
$$




$$
\begin{aligned}
= & \sum_{\epsilon} N_{\epsilon, \epsilon^{\prime}}^{-1} \Omega_{1}\left(\left[Z_{\epsilon}, X\right]\right) \Omega_{1}\left(X_{\epsilon^{\prime}}\right)-\frac{1}{2} \sum_{\epsilon} N_{\epsilon, \epsilon^{\prime}}^{-1} \lambda_{\chi}\left(Z_{\epsilon}\right) \Omega_{1}(X) \Omega_{1}\left(X_{\epsilon^{\prime}}\right) \\
= & \sum_{\epsilon} N_{\epsilon, \epsilon^{\prime}}^{-1} \Omega_{1}\left(\left[X_{\epsilon},[Y, X]\right]\right) \Omega_{1}\left(X_{\epsilon^{\prime}}\right)-\sum_{\epsilon} N_{\epsilon, \epsilon^{\prime}}^{-1} \Omega_{1}\left(\left[Y,\left[X_{\epsilon}, X\right]\right]\right) \Omega_{1}\left(X_{\epsilon^{\prime}}\right) \\
& \quad-\frac{1}{2} \sum_{\epsilon} N_{\epsilon, \epsilon^{\prime}}^{-1}\left\langle X_{\epsilon},\left[Y, X_{\gamma}\right]\right\rangle \Omega_{1}(X) \Omega_{1}\left(X_{\epsilon^{\prime}}\right) \\
= & 2 \Omega_{2}([Y, X])-(1 / 4) d \lambda_{\chi}([Y, X]) \partial_{\gamma}-\sum_{\epsilon} \xi_{\epsilon^{\prime}} \Omega_{1}\left(\left[Y, X_{\gamma}\right]\right) \Omega_{1}\left(X_{\epsilon^{\prime}}\right) \\
& \quad-(1 / 2) \Omega_{1}(X) \Omega_{1}\left(\left[Y, X_{\gamma}\right]\right) \\
= & 2 \Omega_{2}([Y, X])-(1 / 4) d \lambda_{\chi}([Y, X]) \partial_{\gamma}-\Omega_{1}(X) \Omega_{1}\left(\left[Y, X_{\gamma}\right]\right) \\
& \quad-\sum_{\epsilon} \xi_{\epsilon^{\prime}} \Omega_{1}\left(\left[\left[Y, X_{\gamma}\right], X_{\epsilon^{\prime}}\right]\right)-(1 / 2) \Omega_{1}(X) \Omega_{1}\left(\left[Y, X_{\gamma}\right]\right) \\
=2 \Omega_{2}([Y, X])-(1 / 4) d \lambda_{\chi}([Y, X]) \partial_{\gamma}-(3 / 2) \Omega_{1}(X) \Omega_{1}\left(\left[Y, X_{\gamma}\right]\right) & +\sum_{\epsilon} \xi_{\epsilon^{\prime}} \Omega_{1}\left(\left[X_{\gamma},\left[Y, X_{\epsilon^{\prime}}\right]\right]\right) \\
=2 \Omega_{2}([Y, X])-(1 / 4) d \lambda_{\chi}([Y, X]) \partial_{\gamma}-(3 / 2) \Omega_{1}(X) \Omega_{1}\left(\left[Y, X_{\gamma}\right]\right) & \quad-\Omega_{1}\left(\left[[Y, X], X_{\gamma}\right]\right) \\
= & 2 \Omega_{2}([Y, X])-(1 / 4) d \lambda_{\chi}([Y, X]) \partial_{\gamma}-(3 / 2) \Omega_{1}(X) \Omega_{1}\left(\left[Y, X_{\gamma}\right]\right) \\
& \quad-\lambda_{\chi}([Y, X]) \partial_{\gamma} \\
= & 2 \Omega_{2}([Y, X])-(1 / 4)(d+4) \lambda_{\chi}([Y, X]) \partial_{\gamma}-(3 / 2) \Omega_{1}(X) \Omega_{1}\left(\left[Y, X_{\gamma}\right]\right)
\end{aligned}
$$

where we used Lemma 6.11 from the third line to the fourth. Also,

$$
\begin{aligned}
T_{42}= & \sum_{\epsilon} N_{\epsilon, \epsilon^{\prime}}^{-1}\left[\Omega_{1}\left(X_{\epsilon^{\prime}}\right), \Omega_{1}\left(\left[\operatorname{pr}_{0}\left(Z_{\epsilon}\right), X\right]\right)\right] \\
= & \sum_{\epsilon, \mu} N_{\epsilon, \epsilon^{\prime}}^{-1}\left[\Omega_{1}\left(X_{\epsilon^{\prime}}\right), \xi_{\mu} \Omega_{1}\left(\left[\operatorname{pr}_{0}\left(Z_{\epsilon}\right), X_{\mu}\right]\right)\right] \\
= & \sum_{\epsilon, \mu} N_{\epsilon, \epsilon^{\prime}}^{-1} \xi_{\mu}\left[\Omega_{1}\left(X_{\epsilon^{\prime}}\right), \Omega_{1}\left(\left[\operatorname{pr}_{0}\left(Z_{\epsilon}\right), X_{\mu}\right]\right)\right] \\
& +\sum_{\epsilon, \mu} N_{\epsilon, \epsilon^{\prime}}^{-1}\left[\Omega_{1}\left(X_{\epsilon^{\prime}}\right), \xi_{\mu}\right] \Omega_{1}\left(\left[\operatorname{pr}_{0}\left(Z_{\epsilon}\right), X_{\mu}\right]\right) \\
= & \sum_{\epsilon, \mu} N_{\epsilon, \epsilon^{\prime}}^{-1} \xi_{\mu} \Omega_{1}\left(\left[X_{\epsilon^{\prime}},\left[\operatorname{pr}_{0}\left(Z_{\epsilon}\right), X_{\mu}\right]\right]\right)+\sum_{\epsilon} N_{\epsilon, \epsilon^{\prime}}^{-1} \Omega_{1}\left(\left[\operatorname{pr}_{0}\left(Z_{\epsilon}\right), X_{\epsilon^{\prime}}\right]\right) \\
= & \sum_{\epsilon} N_{\epsilon, \epsilon^{\prime}}^{-1} \Omega_{1}\left(\left[X_{\epsilon^{\prime}},\left[\operatorname{pr}_{0}\left(Z_{\epsilon}\right), X\right]\right]\right)+\sum_{\epsilon} N_{\epsilon, \epsilon^{\prime}}^{-1} \Omega_{1}\left(\left[\operatorname{pr}_{0}\left(Z_{\epsilon}\right), X_{\epsilon^{\prime}}\right]\right)
\end{aligned}
$$


We shall consider these terms separately. The first is

$$
\begin{aligned}
T_{421}= & \sum_{\epsilon} N_{\epsilon, \epsilon^{\prime}}^{-1} \Omega_{1}\left(\left[X_{\epsilon^{\prime}},\left[Z_{\epsilon}, X\right]\right]\right)-\frac{1}{2} \sum_{\epsilon} N_{\epsilon, \epsilon^{\prime}}^{-1} \lambda_{\chi}\left(Z_{\epsilon}\right) \Omega_{1}\left(\left[X_{\epsilon^{\prime}}, X\right]\right) \\
= & \sum_{\epsilon} N_{\epsilon, \epsilon^{\prime}}^{-1} \Omega_{1}\left(\left[X_{\epsilon^{\prime}},\left[X_{\epsilon},[Y, X]\right]\right]\right)-\sum_{\epsilon} N_{\epsilon, \epsilon^{\prime}}^{-1} \Omega_{1}\left(\left[X_{\epsilon^{\prime}},\left[Y,\left[X_{\epsilon}, X\right]\right]\right]\right) \\
& -\frac{1}{2} \sum_{\epsilon} N_{\epsilon, \epsilon^{\prime}}^{-1}\left\langle X_{\epsilon},\left[Y, X_{\gamma}\right]\right\rangle \Omega_{1}\left(\left[X_{\epsilon^{\prime}}, X\right]\right) \\
= & (1 / 2) d \lambda_{\chi}([Y, X]) \partial_{\gamma}-\sum_{\epsilon} \xi_{\epsilon^{\prime}} \Omega_{1}\left(\left[X_{\epsilon^{\prime}},\left[Y, X_{\gamma}\right]\right]\right) \\
& -(1 / 2) \Omega_{1}\left(\left[\left[Y, X_{\gamma}\right], X\right]\right) \\
= & (1 / 2) d \lambda_{\chi}([Y, X]) \partial_{\gamma}-\Omega_{1}\left(\left[X,\left[Y, X_{\gamma}\right]\right]\right)-(1 / 2) \Omega_{1}\left(\left[\left[Y, X_{\gamma}\right], X\right]\right) \\
= & (1 / 2) d \lambda_{\chi}([Y, X]) \partial_{\gamma}-(1 / 2) \Omega_{1}\left(\left[X,\left[Y, X_{\gamma}\right]\right]\right) \\
= & (1 / 2) d \lambda_{\chi}([Y, X]) \partial_{\gamma}-(1 / 2) \Omega_{1}\left(\left[[X, Y], X_{\gamma}\right]\right) \\
= & (1 / 2) d \lambda_{\chi}([Y, X]) \partial_{\gamma}+(1 / 2) \lambda_{\chi}([Y, X]) \partial_{\gamma} \\
= & (1 / 2)(d+1) \lambda_{\chi}([Y, X]) \partial_{\gamma},
\end{aligned}
$$

where we have used Lemma 6.6 from the second line to the third. This completes the evaluation of the first term in $T_{42}$. The second term is

$$
\begin{aligned}
T_{422} & =\sum_{\epsilon} N_{\epsilon, \epsilon^{\prime}}^{-1} \Omega_{1}\left(\left[\operatorname{pr}_{0}\left(Z_{\epsilon}\right), X_{\epsilon^{\prime}}\right]\right) \\
& =\sum_{\epsilon} N_{\epsilon, \epsilon^{\prime}}^{-1} \Omega_{1}\left(\left[\left[X_{\epsilon}, Y\right], X_{\epsilon^{\prime}}\right]\right)-\frac{1}{2} \sum_{\epsilon} N_{\epsilon, \epsilon^{\prime}}^{-1} \lambda_{\chi}\left(Z_{\epsilon}\right) \Omega_{1}\left(X_{\epsilon^{\prime}}\right) \\
& =-(1 / 2) d \Omega_{1}\left(\left[Y, X_{\gamma}\right]\right)-\frac{1}{2} \sum_{\epsilon} N_{\epsilon, \epsilon^{\prime}}^{-1}\left\langle X_{\epsilon},\left[Y, X_{\gamma}\right]\right\rangle \Omega_{1}\left(X_{\epsilon^{\prime}}\right) \\
& =-(1 / 2) d \Omega_{1}\left(\left[Y, X_{\gamma}\right]\right)-(1 / 2) \Omega_{1}\left(\left[Y, X_{\gamma}\right]\right) \\
& =-(1 / 2)(d+1) \Omega_{1}\left(\left[Y, X_{\gamma}\right]\right),
\end{aligned}
$$

where we used Lemma 6.5 from the second line to the third. We conclude that

$$
T_{42}=(1 / 2)(d+1)\left(\lambda_{\chi}([Y, X]) \partial_{\gamma}-\Omega_{1}\left(\left[Y, X_{\gamma}\right]\right)\right) .
$$

By adding the evaluations of $T_{41}$ and $T_{42}$ we obtain

$$
\begin{aligned}
T_{4}=2 \Omega_{2}([Y, X])+(1 / 4)(d-2) \lambda_{\chi}([Y, X]) \partial_{\gamma}-(1 / 2)(d+1) \Omega_{1}\left(\left[Y, X_{\gamma}\right]\right) \\
\\
-(3 / 2) \Omega_{1}(X) \Omega_{1}\left(\left[Y, X_{\gamma}\right]\right) .
\end{aligned}
$$

All that remains to obtain the proposed formula for $\left[D_{-\gamma}, \tilde{\Omega}_{3}(Y)\right]$ is to add $T_{1}$, $T_{2}, T_{3}$, and $s-s_{2}$ times $T_{4}$, and to simplify the coefficient of $\Omega_{2}([Y, X])$ that 
results. This coefficient is

$$
\begin{aligned}
& 1-(1 / 2) p(\mathfrak{g})+s+2\left(s-s_{2}\right) \\
& =3 s-(1 / 2) p(\mathfrak{g})-c(\mathfrak{g})+3 \\
& =3 s-(1 / 2)(d+4)+3 \\
& =3 s-(1 / 2)(d-2) \\
& =3\left(s-s_{3}\right) .
\end{aligned}
$$

In this computation, we used the fact that, by Proposition 3.1, $c(\mathfrak{g})+(1 / 2) p(\mathfrak{g})=$ $(1 / 2)(d+4)$, as well as the definition $s_{2}=(1 / 2) c(\mathfrak{g})-1$. This completes the proof of the formula for $\left[D_{-\gamma}, \tilde{\Omega}_{3}(Y)\right]$.

The last step in the proof is to use the result of Proposition 6.4 and the above evaluation to determine $\left[D_{-\gamma}, \Omega_{3}(Y)\right]$. Recall that

$$
\begin{array}{r}
{\left[D_{-\gamma}, C_{3}(Y)\right]=-3 \xi_{\gamma} C_{3}(Y)+C_{3}\left(\left[\tau_{2}(X), Y\right]\right)+s \lambda_{\chi}([Y, X]) \partial_{\gamma}} \\
-(2 s+1) \Omega_{1}\left(\left[Y, X_{\gamma}\right]\right)-\Omega_{1}(X) \Omega_{1}\left(\left[Y, X_{\gamma}\right]\right)
\end{array}
$$

so that only the coefficients of $\lambda_{\chi}([Y, X]) \partial_{\gamma}, \Omega_{1}\left(\left[Y, X_{\gamma}\right]\right)$, and $\Omega_{1}(X) \Omega_{1}\left(\left[Y, X_{\gamma}\right]\right)$ in $\left[D_{-\gamma}, \Omega_{3}(Y)\right]$ require further attention. We first note that $2 c(\mathfrak{g})+p(\mathfrak{g})=d+4$, so that $c(\mathfrak{g})-p(\mathfrak{g})=3 c(\mathfrak{g})-d-4$. Thus we may write

$$
\Omega_{3}(Y)=(1 / 3) \tilde{\Omega}_{3}(Y)+(1 / 12)(3 c(\mathfrak{g})-d-4) C_{3}(Y) .
$$

The coefficient of $\lambda_{\chi}([Y, X]) \partial_{\gamma}$ in $\left[D_{-\gamma}, \Omega_{3}(Y)\right]$ is therefore

$$
\begin{aligned}
& (1 / 12)\left(s-s_{2}\right)(d-2)+(1 / 12)(3 c(\mathfrak{g})-d-4) s \\
& =(1 / 12)\left((3 c(\mathfrak{g})-6) s-s_{2}(d-2)\right) \\
& =(1 / 12)(3(c(\mathfrak{g})-2) s-(1 / 2)(c(\mathfrak{g})-2)(d-2)) \\
& =(1 / 12)(c(\mathfrak{g})-2)(3 s-(1 / 2)(d-2)) \\
& =(1 / 4)(c(\mathfrak{g})-2)\left(s-s_{3}\right) .
\end{aligned}
$$

The coefficient of $\Omega_{1}\left(\left[Y, X_{\gamma}\right]\right)$ in the same commutator is

$$
\begin{aligned}
& -(1 / 6)\left(s-s_{2}\right)(d+1)-(1 / 12)(3 c(\mathfrak{g})-d-4)(2 s+1) \\
& =-(1 / 12)\left(2(d+1)\left(s-s_{2}\right)+(3 c(\mathfrak{g})-d-4)(2 s+1)\right) \\
& =-(1 / 12)((6 c(\mathfrak{g})-6) s-(d+1)(c(\mathfrak{g})-2)+3 c(\mathfrak{g})-d-4) \\
& =-(1 / 12)(6(c(\mathfrak{g})-1) s-c(\mathfrak{g}) d+2 c(\mathfrak{g})+d-2) \\
& =-(1 / 12)(6(c(\mathfrak{g})-1) s-(c(\mathfrak{g})-1)(d-2)) \\
& =-(1 / 2)(c(\mathfrak{g})-1)\left(s-s_{3}\right) .
\end{aligned}
$$


Finally, the coefficient of $\Omega_{1}(X) \Omega_{1}\left(\left[Y, X_{\gamma}\right]\right)$ in the commutator is

$$
\begin{aligned}
& -(1 / 2)\left(s-s_{2}\right)-(1 / 12)(3 c(\mathfrak{g})-d-4) \\
& =-(1 / 12)\left(6 s-6 s_{2}+3 c(\mathfrak{g})-d-4\right) \\
& =-(1 / 12)(6 s-3 c(\mathfrak{g})+6+3 c(\mathfrak{g})-d-4) \\
& =-(1 / 12)(6 s-(d-2)) \\
& =-(1 / 2)\left(s-s_{3}\right) .
\end{aligned}
$$

This completes the proof.

We now begin the construction of $\Omega_{4}$. Let $\mathfrak{l}_{0}=[\mathfrak{l}, \mathfrak{l}]$ and recall that there is a decomposition $\mathfrak{l}_{0}=\oplus_{\mathfrak{e}} \mathfrak{l}(\mathfrak{C})$, where the sum is over the components of $\mathcal{D}_{\gamma}(\mathfrak{g}, \mathfrak{h})$. Each $\mathfrak{l}(\mathcal{C})$ is a simple Lie algebra and also an ideal of $\mathfrak{l}$. Moreover, the restriction of $\mathbf{B}_{\mathfrak{g}}$ to $\mathfrak{l}_{0}$ is non-degenerate and the various $\mathfrak{l}(\mathcal{C})$ are orthogonal to one another with respect to this form. We choose ordered bases of each $\mathfrak{l}(\mathcal{C})$ and concatenate them to obtain an ordered basis $Z_{1}, \ldots, Z_{d}$ of $\mathfrak{l}_{0}$. Let $I(\mathcal{C})=\left\{i \mid Z_{i} \in \mathfrak{l}(\mathcal{C})\right\}$ for each C. Define $b_{i j}=\mathbf{B}_{\mathfrak{g}}\left(Z_{i}, Z_{j}\right)$. The matrix $\left[b_{i j}\right]$ is non-singular and blockdiagonal with blocks indexed by the $\mathcal{C}$. We let its inverse be $\left[b^{i j}\right]$ and define $Z^{i}=\sum_{j=1}^{d} b^{i j} Z_{j}$, so that $Z^{1}, \ldots, Z^{d}$ is dual to $Z_{1}, \ldots, Z_{d}$ with respect to $\mathbf{B}_{\mathfrak{g}}$. Note that if $i \in I(\mathcal{C})$ then $Z^{i} \in \mathfrak{l}(\mathcal{C})$.

Suppose that $F(\cdot, \cdot)$ is a bilinear function on $\mathfrak{l}_{0}$ with values in any $\mathbb{C}$-vector space. Then we may consider the sum

$$
\sum_{i \in I(\mathcal{C})} F\left(Z_{i}, Z^{i}\right)
$$

If we replace $Z_{i}, i \in I(\mathcal{C})$, by a different ordered basis of $\mathfrak{l}(\mathrm{C})$ then there is a compensating change in the dual basis which leaves the sum unchanged. In particular, since duality between bases is a symmetric relation, we have

$$
\sum_{i \in I(\mathcal{C})} F\left(Z_{i}, Z^{i}\right)=\sum_{i \in I(\mathcal{C})} F\left(Z^{i}, Z_{i}\right)
$$

We shall have recourse to this observation on several occasions below.

For $Z \in \mathfrak{l}$ we introduce structure constants $c_{i}^{j}(Z)$ by requiring that

$$
\left[Z, Z_{i}\right]=\sum_{j=1}^{d} c_{i}^{j}(Z) Z_{j}
$$

Observe that if $i \in I(\mathcal{C})$ then $c_{i}^{j}(Z)=0$ unless $j \in I(\mathcal{C})$ also; this is another expression of the fact that $\mathfrak{l}(\mathcal{C})$ is an ideal of $\mathfrak{l}$. Thus the definition may also be 
written as

$$
\left[Z, Z_{i}\right]=\sum_{j \in I\left(\mathcal{C}_{i}\right)} c_{i}^{j}(Z) Z_{j}
$$

where $\mathcal{C}_{i}$ denotes the component such that $Z_{i} \in \mathfrak{l}\left(\mathcal{C}_{i}\right)$. The invariance of $\mathbf{B}_{\mathfrak{g}}$ implies the relation

$$
\left[Z, Z^{i}\right]=-\sum_{j \in I\left(\mathfrak{C}_{i}\right)} c_{j}^{i}(Z) Z^{j}
$$

for all $Z \in \mathfrak{l}$.

Lemma 6.12. If $\mathcal{C}$ is a component of $\mathcal{D}_{\gamma}(\mathfrak{g}, \mathfrak{h})$ then

$$
\sum_{i \in I(\mathcal{C})}\left[Z_{i}, Z^{i}\right]=0 .
$$

Proof. It follows from the discussion of bilinear sums that

$$
\sum_{i \in I(\mathcal{C})}\left[Z_{i}, Z^{i}\right]=\sum_{i \in I(\mathfrak{C})}\left[Z^{i}, Z_{i}\right]=-\sum_{i \in I(\mathfrak{C})}\left[Z_{i}, Z^{i}\right]
$$

This implies the claim.

Proposition 6.5. Let $\mathcal{C}$ be a component of $\mathcal{D}_{\gamma}(\mathfrak{g}, \mathfrak{h})$. Then there is a constant $\Lambda(\mathcal{C})$ such that

$$
\sum_{i \in I(\mathcal{C})}\left[Z_{i},\left[Z^{i}, Y\right]\right]=\Lambda(\mathcal{C}) Y
$$

for all $Y \in V^{+}$.

Proof. Suppose first that $\mathfrak{g}$ is not of type $\mathrm{A}_{r}$. Then $V^{+}$is an irreducible representation of $\mathfrak{l}$ and hence of $\mathfrak{l}_{0}$. From the decomposition $\mathfrak{l}_{0}=\oplus_{\mathfrak{e}} \mathfrak{l}(\mathcal{C})$ of $\mathfrak{l}_{0}$ into ideals, we conclude that the restriction of $V^{+}$to $\mathfrak{l}(\mathcal{C})$ is isotypic. The constant $\Lambda(\mathcal{C})$ is simply the value of the infinitesimal character of this type on the Casimir element $\sum_{i \in I(\mathcal{C})} Z_{i} Z^{i} \in \mathcal{U}(\mathfrak{l}(\mathcal{C}))$.

Now suppose that $\mathfrak{g}$ is of type $\mathrm{A}_{r}$. The representation $V^{+}$is the direct sum of an irreducible representation of $\mathfrak{l}_{0}$ and the dual of that representation. By repeating the argument of the previous paragraph we conclude that the restriction of $V^{+}$to $\mathfrak{l}(\mathcal{C})$ is the sum of two isotypic representations, with dual types. Since the Casimir element in question is of even degree, the infinitesimal characters of an irreducible representation and of its dual take the same value on this element. That common value is $\Lambda(\mathcal{C})$ in this case. 
Corollary 6.1. Let $\mathcal{C}$ be a component of $\mathcal{D}_{\gamma}(\mathfrak{g}, \mathfrak{h})$. Then for all $\epsilon, \tau \in$ $R\left(V^{+}\right)$we have

$$
\sum_{i \in I(\mathcal{C})} \sum_{\mu \in R\left(V^{+}\right)} M_{\epsilon \mu}\left(Z^{i}\right) M_{\mu \tau}\left(Z_{i}\right)=\Lambda(\mathcal{C}) \kappa_{\epsilon \tau} .
$$

Proof. This follows immediately by taking $Y=X_{\epsilon}$ in Proposition 6.5, expanding the resulting brackets using the matrix coefficients $M$, and then comparing the members of the resulting identity.

For the next result, recall that we are assuming that $\mathfrak{g}$ is not of type $\mathrm{C}_{r}$, so that a simple root $\delta \in R\left(V^{+}\right)$having the property described in Section 3 exists. We use the notation from that section in the proof.

Proposition 6.6. Let $\mathcal{C}$ be a component of $\mathcal{D}_{\gamma}(\mathfrak{g}, \mathfrak{h})$ and $\langle\mathcal{C}\rangle \subset \mathfrak{h}^{*}(\mathbb{R})$ denote the $\mathbb{R}$-span of the simple roots whose nodes in the Dynkin diagram lie in $\mathrm{C}$. Then

$$
\Lambda(\mathcal{C})=\left\|\operatorname{pr}_{\langle\mathcal{C}\rangle}(\delta)\right\|^{2}+(1 / 2)\left(\operatorname{dim}\left(V^{+}\right)-2\right),
$$

where $\operatorname{pr}_{\langle\mathrm{e}\rangle}$ denotes the orthogonal projection onto the subspace $\langle\mathrm{C}\rangle$.

Proof. It will be convenient to think of $\mathcal{C}$ as being a set of simple roots, by identifying simple roots with their nodes in the Dynkin diagram. Recall that $R(\mathfrak{l}, \mathcal{C})$ denotes the set of roots in $R(\mathfrak{l})$ that are combinations of simple roots in $\mathrm{C}$. Let $R^{+}(\mathfrak{l}, \mathcal{C})=R^{+} \cap R(\mathfrak{l}, \mathcal{C})$. The set

$$
\left\{H_{\alpha} \mid \alpha \in \mathcal{C}\right\} \cup\left\{X_{\alpha}, X_{-\alpha} \mid \alpha \in R^{+}(\mathfrak{l}, \mathfrak{C})\right\}
$$

is a Chevalley basis for the algebra $\mathfrak{l}(\mathcal{C})$. The form $\mathbf{B}_{\mathfrak{g}}$ is normalized in such a way that $\mathbf{B}_{\mathfrak{g}}\left(X_{\alpha}, X_{-\alpha}\right)=2 /\|\alpha\|^{2}$ and

$$
g_{\alpha \beta}=\mathbf{B}_{\mathfrak{g}}\left(H_{\alpha}, H_{\beta}\right)=\frac{4(\alpha, \beta)}{\|\alpha\|^{2}\|\beta\|^{2}}
$$

for $\alpha, \beta \in \mathcal{C}$. Let $\left[g^{\alpha \beta}\right]$ be the inverse of $\left[g_{\alpha \beta}\right]$. The element dual to $H_{\alpha}$ is then

$$
H^{\alpha}=\sum_{\beta \in \mathcal{C}} g^{\alpha \beta} H_{\beta},
$$

the element dual to $X_{\alpha}$ is $X^{\alpha}=\left(\|\alpha\|^{2} / 2\right) X_{-\alpha}$, and the element dual to $X_{-\alpha}$ is $X^{-\alpha}=\left(\|\alpha\|^{2} / 2\right) X_{\alpha}$. 
In order to evaluate $\Lambda(\mathcal{C})$, we take $Y=X_{\delta}$ in Proposition 6.5 and consider the contribution to the sum from the three parts of the basis separately. From $\left\{H_{\alpha}\right\}$, we obtain

$$
\begin{aligned}
\sum_{\alpha \in \mathcal{C}}\left[H_{\alpha},\left[H^{\alpha}, X_{\delta}\right]\right] & =\sum_{\alpha, \beta \in \mathcal{C}} g^{\alpha \beta}\left[H_{\alpha},\left[H_{\beta}, X_{\delta}\right]\right] \\
& =\sum_{\alpha, \beta \in \mathcal{C}} g^{\alpha \beta} \delta\left(H_{\alpha}\right) \delta\left(H_{\beta}\right) X_{\delta} \\
& =\sum_{\alpha, \beta \in \mathcal{C}} g^{\alpha \beta} \frac{4(\alpha, \delta)(\delta, \beta)}{\|\alpha\|^{2}\|\beta\|^{2}} X_{\delta} .
\end{aligned}
$$

Let us write $\check{\alpha}=2 \alpha /\|\alpha\|^{2}$. Then $g_{\alpha \beta}=(\check{\alpha}, \check{\beta})$ and the coefficient of $X_{\delta}$ in the above is

$$
\sum_{\alpha, \beta \in \mathrm{C}} g^{\alpha \beta}(\check{\alpha}, \delta)(\delta, \check{\beta})
$$

which is precisely $\left\|\operatorname{pr}_{\langle\mathcal{C}\rangle}(\delta)\right\|^{2}$.

Now let $\alpha \in R^{+}(\mathfrak{l}, \mathfrak{C})$ and suppose that $\tau=\delta \pm \alpha$ is a root. We have $(\gamma, \tau)=(\gamma, \delta)=1$ and so $\tau \in R\left(V^{+}\right)$. In particular, $\tau$ is a positive root. Since $\tau-\delta= \pm \alpha$ is a root, $\tau \in S(\delta)$ and so $(\tau, \delta)=1$. It follows from this that $s_{\delta}(\tau)=\tau-\delta= \pm \alpha$. Now $\tau \neq \delta$ and $\delta$ is a simple root. Thus $s_{\delta}(\tau)$ is a positive root. It follows from this discussion that $\delta-\alpha$ is never a root and $\tau=\delta+\alpha$ is a root precisely when $\tau \in S(\delta)$, in which case $\alpha=\tau-\delta$. Thus

$$
\sum_{\alpha \in R^{+}(\mathfrak{r}, \mathcal{C})}\left[X_{\alpha},\left[X^{\alpha}, X_{\delta}\right]\right]=0
$$

and

$$
\begin{aligned}
\sum_{\alpha \in R^{+}(\mathfrak{l}, \mathcal{C})}\left[X_{-\alpha},\left[X^{-\alpha}, X_{\delta}\right]\right] & =\sum_{\alpha \in R^{+}(\mathfrak{l}, \mathcal{C})}\left(\|\alpha\|^{2} / 2\right)\left[X_{-\alpha},\left[X_{\alpha}, X_{\delta}\right]\right] \\
& =\sum_{\tau \in S(\delta)}\left(\|\tau-\delta\|^{2} / 2\right)\left[X_{\delta-\tau},\left[X_{\tau-\delta}, X_{\delta}\right]\right] \\
& =\sum_{\tau \in S(\delta)}\left(\|\tau\|^{2} / 2\right) N_{\tau-\delta, \delta} N_{\delta-\tau, \tau} X_{\delta} .
\end{aligned}
$$

Now $(\delta-\tau)+\tau+(-\delta)=0$ and applying (C8) and (C9) to this gives

$$
\frac{N_{\delta-\tau, \tau}}{2}=\frac{N_{-\delta, \delta-\tau}}{\|\tau\|^{2}}=\frac{N_{\tau-\delta, \delta}}{\|\tau\|^{2}} .
$$

It follows that the coefficient of $X_{\delta}$ above is

$$
\sum_{\tau \in S(\delta)} N_{\tau-\delta, \delta}^{2}=\sum_{\tau \in S(\delta)}\left(p_{\tau-\delta, \delta}+1\right)^{2}
$$




$$
\begin{aligned}
& =\sum_{\tau \in S(\delta)} 1 \\
& =|S(\delta)| .
\end{aligned}
$$

We have $|S(\delta)|=(1 / 2)\left(\operatorname{dim}\left(V^{+}\right)-2\right)$, and this completes the evaluation of $\Lambda(\mathcal{C})$.

Lemma 6.13. Let $\mathcal{C}$ be a component of $\mathcal{D}_{\gamma}(\mathfrak{g}, \mathfrak{h})$. Then we have

$$
\sum_{i \in I(\mathcal{C})} \sum_{\alpha \in R\left(V^{+}\right)} N_{\alpha, \alpha^{\prime}}^{-1} \Omega_{1}\left(\left[Z_{i}, X_{\alpha^{\prime}}\right]\right) \Omega_{1}\left(\left[Z^{i}, X_{\alpha}\right]\right)=(1 / 2) \operatorname{dim}\left(V^{+}\right) \Lambda(\mathcal{C}) \partial_{\gamma} .
$$

Proof. Let $S$ denote the left-hand side of the proposed identity. This expression depends linearly on the $Z_{i}$ and the $Z^{i}$ and consequently

$$
S=\sum_{i \in I(\mathcal{C})} \sum_{\alpha \in R\left(V^{+}\right)} N_{\alpha, \alpha^{\prime}}^{-1} \Omega_{1}\left(\left[Z^{i}, X_{\alpha^{\prime}}\right]\right) \Omega_{1}\left(\left[Z_{i}, X_{\alpha}\right]\right) .
$$

For compactness, we suppress the domains of summation from now on. By expanding the brackets in the previous expression for $S$ we obtain

$$
\begin{aligned}
S= & \sum_{i, \alpha, \epsilon, \tau} N_{\alpha, \alpha^{\prime}}^{-1} M_{\alpha^{\prime} \epsilon}\left(Z^{i}\right) M_{\alpha \tau}\left(Z_{i}\right) \Omega_{1}\left(X_{\epsilon}\right) \Omega_{1}\left(X_{\tau}\right) \\
= & -\sum_{i, \alpha, \epsilon, \tau} N_{\alpha, \alpha^{\prime}}^{-1} M_{\alpha \epsilon}\left(Z^{i}\right) M_{\alpha^{\prime} \tau}\left(Z_{i}\right) \nabla_{\epsilon} \nabla_{\tau} \\
= & -\sum_{i, \alpha, \epsilon, \tau} N_{\alpha, \alpha^{\prime}}^{-1} M_{\alpha \tau}\left(Z^{i}\right) M_{\alpha^{\prime} \epsilon}\left(Z_{i}\right) \nabla_{\tau} \nabla_{\epsilon} \\
= & -\sum_{i, \alpha, \epsilon, \tau} N_{\alpha, \alpha^{\prime}}^{-1} M_{\alpha \tau}\left(Z^{i}\right) M_{\alpha^{\prime} \epsilon}\left(Z_{i}\right) \nabla_{\epsilon} \nabla_{\tau} \\
& -\sum_{i, \alpha, \epsilon, \tau} N_{\alpha, \alpha^{\prime}}^{-1} M_{\alpha \tau}\left(Z^{i}\right) M_{\alpha^{\prime} \epsilon}\left(Z_{i}\right)\left[\nabla_{\tau}, \nabla_{\epsilon}\right] \\
= & -\sum_{i, \alpha} N_{\alpha, \alpha^{\prime}}^{-1} \Omega_{1}\left(\left[Z_{i}, X_{\alpha^{\prime}}\right]\right) \Omega_{1}\left(\left[Z^{i}, X_{\alpha}\right]\right) \\
& -\sum_{i, \alpha, \epsilon, \tau} N_{\alpha, \alpha^{\prime}}^{-1} M_{\alpha \tau}\left(Z^{i}\right) M_{\alpha^{\prime} \epsilon}\left(Z_{i}\right) N_{\tau, \tau^{\prime}} \kappa_{\tau \epsilon^{\prime}} \partial_{\gamma} \\
= & -S-\sum_{i, \alpha, \tau} N_{\alpha, \alpha^{\prime}}^{-1} N_{\tau, \tau^{\prime}} M_{\alpha \tau}\left(Z^{i}\right) M_{\alpha^{\prime} \tau^{\prime}}\left(Z_{i}\right) \partial_{\gamma} .
\end{aligned}
$$

In this computation, we replaced $\alpha$ by $\alpha^{\prime}$, and then interchanged $\epsilon$ and $\tau$. The rest of the computation should be self-explanatory. Thus

$$
S=-\frac{1}{2} \sum_{i, \alpha, \tau} N_{\alpha, \alpha^{\prime}}^{-1} N_{\tau, \tau^{\prime}} M_{\alpha \tau}\left(Z^{i}\right) M_{\alpha^{\prime} \tau^{\prime}}\left(Z_{i}\right) \partial_{\gamma}
$$




$$
\begin{aligned}
& =-\frac{1}{2} \sum_{i, \alpha, \tau} N_{\tau^{\prime}, \tau}^{-1} N_{\tau, \tau^{\prime}} M_{\alpha \tau}\left(Z^{i}\right) M_{\tau \alpha}\left(Z_{i}\right) \partial_{\gamma} \\
& =\frac{1}{2} \sum_{i, \alpha, \tau} M_{\alpha \tau}\left(Z^{i}\right) M_{\tau \alpha}\left(Z_{i}\right) \partial_{\gamma} \\
& =\frac{1}{2} \sum_{\alpha} \kappa_{\alpha \alpha} \Lambda(\mathcal{C}) \partial_{\gamma} \\
& =(1 / 2) \operatorname{dim}\left(V^{+}\right) \Lambda(\mathcal{C}) \partial_{\gamma} .
\end{aligned}
$$

In this computation, we used the symmetry $N_{\alpha, \alpha^{\prime}}^{-1} M_{\alpha^{\prime} \tau^{\prime}}\left(Z_{i}\right)=N_{\tau^{\prime}, \tau}^{-1} M_{\tau \alpha}\left(Z_{i}\right)$ and then appealed to Corollary 6.1.

Let $\mathcal{C}$ be a component of $\mathcal{D}_{\gamma}(\mathfrak{g}, \mathfrak{h})$. The polynomial

$$
Y \mapsto \sum_{i \in I(\mathcal{C})} \mathbf{B}_{\mathfrak{g}}\left(\tau_{2}(Y), Z_{i}\right) \mathbf{B}_{\mathfrak{g}}\left(\tau_{2}(Y), Z^{i}\right)
$$

on $V^{+}$is a relative invariant associated to the character $\chi^{2}$ and is hence proportional to $\Delta$. Recall that the operator $\Omega_{2}(Z)$ is modeled on the polynomial $Y \mapsto \mathbf{B}_{\mathfrak{g}}\left(\tau_{2}(Y), Z\right)$. This suggests that we define $\Omega_{4}^{\prime}(\mathcal{C}) \in \mathbb{D}[\mathfrak{n}]$ by

$$
\Omega_{4}^{\prime}(\mathcal{C})=\sum_{i \in I(\mathcal{C})}\left\ulcorner\Omega_{2}\left(Z_{i}\right) \Omega_{2}\left(Z^{i}\right)\right\urcorner .
$$

By the discussion of bilinear sums, we have

$$
\sum_{i \in I(\mathcal{C})} \Omega_{2}\left(Z_{i}\right) \Omega_{2}\left(Z^{i}\right)=\sum_{i \in I(\mathcal{C})} \Omega_{2}\left(Z^{i}\right) \Omega_{2}\left(Z_{i}\right) .
$$

It follows that the symmetrization in (6.2) is unnecessary.

Recall that $l \cdot \Omega_{2}(Z)=\chi(l) \Omega_{2}(\operatorname{Ad}(l) Z)$ for $l \in L$ and $Z \in \mathfrak{l}$. Therefore, for $l \in L$,

$$
\begin{aligned}
l \cdot \Omega_{4}^{\prime}(\mathcal{C}) & =\sum_{i \in I(\mathcal{C})}\left(l \cdot \Omega_{2}\left(Z_{i}\right)\right)\left(l \cdot \Omega_{2}\left(Z^{i}\right)\right) \\
& =\chi(l)^{2} \sum_{i \in I(\mathcal{C})} \Omega_{2}\left(\operatorname{Ad}(l) Z_{i}\right) \Omega_{2}\left(\operatorname{Ad}(l) Z^{i}\right) \\
& =\chi(l)^{2} \Omega_{4}^{\prime}(\mathcal{C}),
\end{aligned}
$$

since the ordered bases $\operatorname{Ad}(l) Z_{i}$ and $\operatorname{Ad}(l) Z^{i}, i \in I(\mathcal{C})$, are dual to one another.

Lemma 6.14. We have

$$
\left[D_{-\gamma}, \partial_{\gamma}^{2}\right]=-4 \xi_{\gamma} \partial_{\gamma}^{2}-2 \Omega_{1}(X) \partial_{\gamma}-(4 s+2) \partial_{\gamma}
$$


Proof. We have

$$
\left[D_{-\gamma}, \partial_{\gamma}\right]=-\left[\Pi_{s}\left(X_{-\gamma}\right), \Pi_{s}\left(X_{\gamma}\right)\right]=\Pi_{s}\left(H_{\gamma}\right)
$$

From Lemma $4.2, \Pi_{s}\left(H_{\gamma}\right)=-\partial_{\left[H_{\gamma}, \cdot\right]}-2 s$. Since $H_{\gamma}$ acts as 1 on $V^{+}$and as 2 on $\mathfrak{g}_{\gamma}$, this gives

$$
\left[D_{-\gamma}, \partial_{\gamma}\right]=-2 \xi_{\gamma} \partial_{\gamma}-\Omega_{1}(X)-2 s
$$

The stated value of $\left[D_{-\gamma}, \partial_{\gamma}^{2}\right]$ follows easily from this.

We define

$$
\Omega_{4}(\mathcal{C})=\Omega_{4}^{\prime}(\mathcal{C})+(1 / 2)\left(s-s_{2}(\mathcal{C})\right) \Lambda(\mathcal{C}) \partial_{\gamma}^{2},
$$

where $s_{2}(\mathcal{C})=(1 / 2) c(\mathfrak{g}, \mathcal{C})-1$. Note that $l \cdot \partial_{\gamma}^{2}=\chi(l)^{2} \partial_{\gamma}^{2}$ for all $l \in L$, and so $\Omega_{4}(\mathcal{C})$ enjoys the same transformation law under $L$ that $\Omega_{4}^{\prime}(\mathcal{C})$ does.

Theorem 6.2. $\quad$ Let $\mathcal{C}$ be a component of $\mathcal{D}_{\gamma}(\mathfrak{g}, \mathfrak{h})$. Then we have

$$
\begin{gathered}
{\left[D_{-\gamma}, \Omega_{4}(\mathcal{C})\right]=-4 \xi_{\gamma} \Omega_{4}(\mathcal{C})+2\left(s-s_{4}\right) \sum_{i \in I(\mathcal{C})} \Omega_{1}\left(\left[Z_{i}, X\right]\right) \Omega_{2}\left(Z^{i}\right)} \\
-2 \Lambda(\mathcal{C})\left(s-s_{2}(\mathcal{C})\right)\left(s-s_{4}\right) \partial_{\gamma}
\end{gathered}
$$

where $s_{2}(\mathcal{C})=(1 / 2) c(\mathfrak{g}, \mathcal{C})-1$ and $s_{4}=\left(\operatorname{dim}\left(V^{+}\right)-2\right) / 4$.

Proof. We begin by considering the commutator of $D_{-\gamma}$ and $\Omega_{4}^{\prime}(\mathcal{C})$. This commutator is given by

$$
\left[D_{-\gamma}, \Omega_{4}^{\prime}(\mathcal{C})\right]=\sum_{i \in I(\mathcal{C})}\left[D_{-\gamma}, \Omega_{2}\left(Z_{i}\right)\right] \Omega_{2}\left(Z^{i}\right)+\sum_{i \in I(\mathcal{C})} \Omega_{2}\left(Z_{i}\right)\left[D_{-\gamma}, \Omega_{2}\left(Z^{i}\right)\right]
$$

By Theorem 5.2,

$$
\left[D_{-\gamma}, \Omega_{2}(Z)\right]=-2 \xi_{\gamma} \Omega_{2}(Z)+\Omega_{2}\left(\left[\tau_{2}(X), Z\right]\right)+\left(s-s_{2}(\mathcal{C})\right) \Omega_{1}([Z, X])
$$

for all $Z \in \mathfrak{l}(\mathcal{C})$. We shall consider in turn the contributions of each of the three terms in this formula to $(6.4)$.

The contribution to (6.4) of the first terms in the commutator formulas for $\Omega_{2}$ is

$$
T_{1}=-2 \xi_{\gamma} \Omega_{4}^{\prime}(\mathcal{C})-2 \sum_{i \in I(\mathcal{C})} \Omega_{2}\left(Z_{i}\right) \xi_{\gamma} \Omega_{2}\left(Z^{i}\right)
$$




$$
\begin{aligned}
& =-4 \xi_{\gamma} \Omega_{4}^{\prime}(\mathcal{C})-2 \sum_{i \in I(\mathcal{C})}\left[\Omega_{2}\left(Z_{i}\right), \xi_{\gamma}\right] \Omega_{2}\left(Z^{i}\right) \\
& =-4 \xi_{\gamma} \Omega_{4}^{\prime}(\mathcal{C})+\sum_{i \in I(\mathcal{C})} \Omega_{1}\left(\left[Z_{i}, X\right]\right) \Omega_{2}\left(Z^{i}\right),
\end{aligned}
$$

by Lemma 6.10 .

The contribution from the second terms in the commutator formulas for $\Omega_{2}$ is

$$
\begin{aligned}
T_{2} & =\sum_{i \in I(\mathcal{C})} \Omega_{2}\left(\left[\tau_{2}(X), Z_{i}\right]\right) \Omega_{2}\left(Z^{i}\right)+\sum_{i \in I(\mathcal{C})} \Omega_{2}\left(Z_{i}\right) \Omega_{2}\left(\left[\tau_{2}(X), Z^{i}\right]\right) \\
& =\sum_{i, j \in I(\mathcal{C})} c_{i}^{j}\left(\tau_{2}(X)\right) \Omega_{2}\left(Z_{j}\right) \Omega_{2}\left(Z^{i}\right)-\sum_{i, j \in I(\mathcal{C})} \Omega_{2}\left(Z_{i}\right) c_{j}^{i}\left(\tau_{2}(X)\right) \Omega_{2}\left(Z^{j}\right) \\
& =\sum_{i, j \in I(\mathcal{C})} c_{j}^{i}\left(\tau_{2}(X)\right) \Omega_{2}\left(Z_{i}\right) \Omega_{2}\left(Z^{j}\right)-\sum_{i, j \in I(\mathcal{C})} \Omega_{2}\left(Z_{i}\right) c_{j}^{i}\left(\tau_{2}(X)\right) \Omega_{2}\left(Z^{j}\right) \\
& =-\sum_{i, j \in I(\mathcal{C})}\left[\Omega_{2}\left(Z_{i}\right), c_{j}^{i}\left(\tau_{2}(X)\right)\right] \Omega_{2}\left(Z^{j}\right) .
\end{aligned}
$$

In order to simplify this, we consider the coefficient of $\Omega_{2}\left(Z^{j}\right)$ separately. By using the expression

$$
\Omega_{2}\left(Z_{i}\right)=\frac{1}{2} \sum_{\alpha, \beta \in R\left(V^{+}\right)} N_{\beta, \beta^{\prime}}^{-1} M_{\beta^{\prime} \alpha}\left(Z_{i}\right) \Omega_{1}\left(X_{\beta}\right) \Omega_{1}\left(X_{\alpha}\right)
$$

we get

$$
\begin{aligned}
{\left[\Omega_{2}\left(Z_{i}\right), c_{j}^{i}\left(\tau_{2}(X)\right)\right]=} & \frac{1}{2} \sum_{\alpha, \beta} N_{\beta, \beta^{\prime}}^{-1} M_{\beta^{\prime} \alpha}\left(Z_{i}\right)\left[\Omega_{1}\left(X_{\beta}\right) \Omega_{1}\left(X_{\alpha}\right), c_{j}^{i}\left(\tau_{2}(X)\right)\right] \\
= & \frac{1}{2} \sum_{\alpha, \beta} N_{\beta, \beta^{\prime}}^{-1} M_{\beta^{\prime} \alpha}\left(Z_{i}\right) \Omega_{1}\left(X_{\beta}\right) c_{j}^{i}\left(\partial_{\alpha} \bullet \tau_{2}(X)\right) \\
& +\frac{1}{2} \sum_{\alpha, \beta} N_{\beta, \beta^{\prime}}^{-1} M_{\beta^{\prime} \alpha}\left(Z_{i}\right) c_{j}^{i}\left(\partial_{\beta} \bullet \tau_{2}(X)\right) \Omega_{1}\left(X_{\alpha}\right) \\
= & \frac{1}{2} \sum_{\alpha, \beta} N_{\alpha, \alpha^{\prime}}^{-1} M_{\alpha^{\prime} \beta}\left(Z_{i}\right) c_{j}^{i}\left(\partial_{\alpha} \bullet \tau_{2}(X)\right) \Omega_{1}\left(X_{\beta}\right) \\
& +\frac{1}{2} \sum_{\alpha, \beta} N_{\beta, \beta^{\prime}}^{-1} M_{\beta^{\prime} \alpha}\left(Z_{i}\right) c_{j}^{i}\left(\partial_{\beta} \bullet \tau_{2}(X)\right) \Omega_{1}\left(X_{\alpha}\right) \\
& +\frac{1}{2} \sum_{\alpha, \beta} N_{\alpha, \alpha^{\prime}}^{-1} M_{\alpha^{\prime} \beta}\left(Z_{i}\right) c_{j}^{i}\left(\partial_{\alpha \beta} \bullet \tau_{2}(X)\right) \\
= & \sum_{\alpha, \beta} N_{\alpha, \alpha^{\prime}}^{-1} M_{\alpha^{\prime} \beta}\left(Z_{i}\right) c_{j}^{i}\left(\partial_{\alpha} \bullet \tau_{2}(X)\right) \Omega_{1}\left(X_{\beta}\right)
\end{aligned}
$$




$$
+\frac{1}{2} \sum_{\alpha, \beta} N_{\alpha, \alpha^{\prime}}^{-1} M_{\alpha^{\prime} \beta}\left(Z_{i}\right) c_{j}^{i}\left(\partial_{\alpha \beta} \bullet \tau_{2}(X)\right),
$$

where we have used the symmetry $N_{\beta, \beta^{\prime}}^{-1} M_{\beta^{\prime} \alpha}\left(Z_{i}\right)=N_{\alpha, \alpha^{\prime}}^{-1} M_{\alpha^{\prime} \beta}\left(Z_{i}\right)$ from the second equality to the third, and then interchanged $\alpha$ and $\beta$ in one term from the third to the fourth. By Lemma 3.6 we have

$$
\partial_{\alpha} \bullet \tau_{2}(X)=(1 / 2)\left\langle X_{\alpha}, X\right\rangle H_{\gamma}+N_{\alpha,-\gamma}\left[X, X_{-\alpha^{\prime}}\right]
$$

and

$$
\partial_{\alpha \beta} \bullet \tau_{2}(X)=(1 / 2)\left\langle X_{\alpha}, X_{\beta}\right\rangle H_{\gamma}+N_{\alpha,-\gamma}\left[X_{\beta}, X_{-\alpha^{\prime}}\right] .
$$

Since $c_{j}^{i}$ vanishes on the center of $\mathfrak{l}$, this gives

$$
\begin{aligned}
{\left[\Omega_{2}\left(Z_{i}\right), c_{j}^{i}\left(\tau_{2}(X)\right)\right]=} & \sum_{\alpha, \beta} N_{\alpha, \alpha^{\prime}}^{-1} M_{\alpha^{\prime} \beta}\left(Z_{i}\right) c_{j}^{i}\left(N_{\alpha,-\gamma}\left[X, X_{-\alpha^{\prime}}\right]\right) \Omega_{1}\left(X_{\beta}\right) \\
& +\frac{1}{2} \sum_{\alpha, \beta} N_{\alpha, \alpha^{\prime}}^{-1} M_{\alpha^{\prime} \beta}\left(Z_{i}\right) c_{j}^{i}\left(N_{\alpha,-\gamma}\left[X_{\beta}, X_{-\alpha^{\prime}}\right]\right) \\
= & -\frac{1}{2} \sum_{\alpha, \beta}\|\alpha\|^{2} M_{\alpha^{\prime} \beta}\left(Z_{i}\right) c_{j}^{i}\left(\left[X, X_{-\alpha^{\prime}}\right]\right) \Omega_{1}\left(X_{\beta}\right) \\
& -\frac{1}{4} \sum_{\alpha, \beta}\|\alpha\|^{2} M_{\alpha^{\prime} \beta}\left(Z_{i}\right) c_{j}^{i}\left(\left[X_{\beta}, X_{-\alpha^{\prime}}\right]\right) \\
= & -\frac{1}{2} \sum_{\alpha}\|\alpha\|^{2} c_{j}^{i}\left(\left[X, X_{-\alpha^{\prime}}\right]\right) \Omega_{1}\left(\left[Z_{i}, X_{\alpha^{\prime}}\right]\right) \\
& -\frac{1}{4} \sum_{\alpha}\|\alpha\|^{2} c_{j}^{i}\left(\left[\left[Z_{i}, X_{\alpha^{\prime}}\right], X_{-\alpha^{\prime}}\right]\right) \\
= & -\frac{1}{2} \sum_{\alpha}\|\alpha\|^{2} c_{j}^{i}\left(\left[X, X_{-\alpha}\right]\right) \Omega_{1}\left(\left[Z_{i}, X_{\alpha}\right]\right) \\
& -\frac{1}{4} \sum_{\alpha}\|\alpha\|^{2} c_{j}^{i}\left(\left[\left[Z_{i}, X_{\alpha}\right], X_{-\alpha}\right]\right),
\end{aligned}
$$

where we have used the identity $N_{\alpha,-\gamma} N_{\alpha, \alpha^{\prime}}^{-1}=-(1 / 2)\|\alpha\|^{2}$ from the first equality to the second.

These equalities imply that $T_{2}=T_{21}+T_{22}$ with

$$
T_{21}=\frac{1}{2} \sum_{i, j \in I(\mathcal{C})} \sum_{\alpha}\|\alpha\|^{2} c_{j}^{i}\left(\left[X, X_{-\alpha}\right]\right) \Omega_{1}\left(\left[Z_{i}, X_{\alpha}\right]\right) \Omega_{2}\left(Z^{j}\right)
$$

and

$$
T_{22}=\frac{1}{4} \sum_{i, j \in I(\mathcal{C})} \sum_{\alpha}\|\alpha\|^{2} c_{j}^{i}\left(\left[\left[Z_{i}, X_{\alpha}\right], X_{-\alpha}\right]\right) \Omega_{2}\left(Z^{j}\right) .
$$


By employing Proposition 2.3, we get

$$
\begin{aligned}
T_{22} & =(1 / 2) c(\mathfrak{g}, \mathcal{C}) \sum_{i, j \in I(\mathcal{C})} c_{j}^{i}\left(Z_{i}\right) \Omega_{2}\left(Z^{j}\right) \\
& =-(1 / 2) c(\mathfrak{g}, \mathcal{C}) \sum_{i \in I(\mathcal{C})} \Omega_{2}\left(\left[Z_{i}, Z^{i}\right]\right) \\
& =0
\end{aligned}
$$

from Lemma 6.12. Now observe that

$$
\sum_{i \in I(\mathcal{C})} c_{j}^{i}\left(\left[X, X_{-\alpha}\right]\right) Z_{i}=\left[\left[X, X_{-\alpha}\right], Z_{j}\right]
$$

and so

$$
\begin{aligned}
T_{2} & =T_{21} \\
& =\frac{1}{2} \sum_{j \in I(\mathcal{C})} \sum_{\alpha}\|\alpha\|^{2} \Omega_{1}\left(\left[\left[\left[X, X_{-\alpha}\right], Z_{j}\right], X_{\alpha}\right]\right) \Omega_{2}\left(Z^{j}\right) \\
& =-\frac{1}{2} \sum_{j \in I(\mathcal{C})} \sum_{\alpha}\|\alpha\|^{2} \Omega_{1}\left(\left[\left[Z_{j},\left[X, X_{-\alpha}\right]\right], X_{\alpha}\right]\right) \Omega_{2}\left(Z^{j}\right) \\
& =-(1 / 2) p(\mathfrak{g}, \mathcal{C}) \sum_{j \in I(\mathcal{C})} \Omega_{1}\left(\left[Z_{j}, X\right]\right) \Omega_{2}\left(Z^{j}\right),
\end{aligned}
$$

where the last equality follows from Proposition 2.4.

We now consider the contribution of the third terms in the commutator formula for $\Omega_{2}$. This contribution is $s-s_{2}(\mathcal{C})$ multiplied by

$$
T_{3}=\sum_{i \in I(\mathcal{C})} \Omega_{1}\left(\left[Z_{i}, X\right]\right) \Omega_{2}\left(Z^{i}\right)+\sum_{i \in I(\mathcal{C})} \Omega_{2}\left(Z_{i}\right) \Omega_{1}\left(\left[Z^{i}, X\right]\right) .
$$

The first term, $T_{31}$, is already in a suitable form, and so we consider the second term, $T_{32}$. It is

$$
\begin{aligned}
T_{32} & =\sum_{i \in I(\mathcal{C})} \Omega_{2}\left(Z_{i}\right) \Omega_{1}\left(\left[Z^{i}, X\right]\right) \\
& =\sum_{i \in I(\mathcal{C})} \Omega_{2}\left(Z^{i}\right) \Omega_{1}\left(\left[Z_{i}, X\right]\right) \\
& =\sum_{i \in I(\mathcal{C})} \Omega_{1}\left(\left[Z_{i}, X\right]\right) \Omega_{2}\left(Z^{i}\right)+\sum_{i \in I(\mathcal{C})}\left[\Omega_{2}\left(Z^{i}\right), \Omega_{1}\left(\left[Z_{i}, X\right]\right)\right] .
\end{aligned}
$$


In this expression, the first term, $T_{321}$, is in a suitable form, and so we consider the second term, $T_{322}$. It is

$$
\begin{aligned}
T_{322}= & \sum_{i \in I(\mathcal{C})} \sum_{\alpha \in R\left(V^{+}\right)}\left[\Omega_{2}\left(Z^{i}\right), \xi_{\alpha} \Omega_{1}\left(\left[Z_{i}, X_{\alpha}\right]\right)\right] \\
= & \sum_{i \in I(\mathcal{C})} \sum_{\alpha \in R\left(V^{+}\right)}\left[\Omega_{2}\left(Z^{i}\right), \xi_{\alpha}\right] \Omega_{1}\left(\left[Z_{i}, X_{\alpha}\right]\right) \\
& +\sum_{i \in I(\mathcal{C})} \sum_{\alpha \in R\left(V^{+}\right)} \xi_{\alpha}\left[\Omega_{2}\left(Z^{i}\right), \Omega_{1}\left(\left[Z_{i}, X_{\alpha}\right]\right)\right] \\
= & \sum_{i \in I(\mathcal{C})} \sum_{\alpha \in R\left(V^{+}\right)} N_{\alpha, \alpha^{\prime}}^{-1} \Omega_{1}\left(\left[Z^{i}, X_{\alpha^{\prime}}\right]\right) \Omega_{1}\left(\left[Z_{i}, X_{\alpha}\right]\right) \\
& +\sum_{i \in I(\mathcal{C})} \sum_{\alpha \in R\left(V^{+}\right)} \xi_{\alpha} \Omega_{1}\left(\left[Z^{i},\left[Z_{i}, X_{\alpha}\right]\right]\right) \partial_{\gamma} \\
= & \sum_{i \in I(\mathcal{C})} \sum_{\alpha \in R\left(V^{+}\right)} N_{\alpha, \alpha^{\prime}}^{-1} \Omega_{1}\left(\left[Z_{i}, X_{\alpha^{\prime}}\right]\right) \Omega_{1}\left(\left[Z^{i}, X_{\alpha}\right]\right) \\
& +\sum_{i \in I(\mathcal{C})} \sum_{\alpha \in R\left(V^{+}\right)} \xi_{\alpha} \Omega_{1}\left(\left[Z_{i},\left[Z^{i}, X_{\alpha}\right]\right]\right) \partial_{\gamma} \\
= & (1 / 2) \operatorname{dim}\left(V^{+}\right) \Lambda(\mathcal{C}) \partial_{\gamma}+\Lambda(\mathcal{C}) \sum_{\alpha \in R\left(V^{+}\right)} \xi_{\alpha} \Omega_{1}\left(X_{\alpha}\right) \partial_{\gamma} \\
= & (1 / 2) \operatorname{dim}\left(V^{+}\right) \Lambda(\mathcal{C}) \partial_{\gamma}+\Lambda(\mathcal{C}) \Omega_{1}(X) \partial_{\gamma} .
\end{aligned}
$$

In this computation, we have used Lemma 6.10 to evaluate $\left[\Omega_{2}\left(Z^{i}\right), \xi_{\alpha}\right]$, Lemma 6.4 to evaluate $\left[\Omega_{2}\left(Z^{i}\right), \Omega_{1}\left(\left[Z_{i}, X_{\alpha}\right]\right)\right]$, the principle about expressions depending linearly on $Z_{i}$ and $Z^{i}$, Lemma 6.13 to evaluate the first sum in the third-last equality, and Proposition 6.5 to evaluate part of the second sum. From these computations, we obtain the evaluation

$$
T_{3}=2 \sum_{i \in I(\mathcal{C})} \Omega_{1}\left(\left[Z_{i}, X\right]\right) \Omega_{2}\left(Z^{i}\right)+(1 / 2) \operatorname{dim}\left(V^{+}\right) \Lambda(\mathcal{C}) \partial_{\gamma}+\Lambda(\mathcal{C}) \Omega_{1}(X) \partial_{\gamma} .
$$

By adding $T_{1}, T_{2}$, and $s-s_{2}(\mathcal{C})$ times $T_{3}$, we obtain the evaluation

$$
\begin{aligned}
& {\left[D_{-\gamma}, \Omega_{4}^{\prime}(\mathcal{C})\right]=} \\
& -4 \xi_{\gamma} \Omega_{4}^{\prime}(\mathcal{C})+\left(2 s+1-(1 / 2) p(\mathfrak{g}, \mathcal{C})-2 s_{2}(\mathcal{C})\right) \sum_{i \in I(\mathcal{C})} \Omega_{1}\left(\left[Z_{i}, X\right]\right) \Omega_{2}\left(Z^{i}\right) \\
& \quad+(1 / 2)\left(s-s_{2}(\mathcal{C})\right) \operatorname{dim}\left(V^{+}\right) \Lambda(\mathcal{C}) \partial_{\gamma}+\left(s-s_{2}(\mathcal{C})\right) \Lambda(\mathcal{C}) \Omega_{1}(X) \partial_{\gamma} .
\end{aligned}
$$

Now

$$
1-(1 / 2) p(\mathfrak{g}, \mathcal{C})-2 s_{2}(\mathcal{C})=1-(1 / 2) p(\mathfrak{g}, \mathcal{C})-c(\mathfrak{g}, \mathcal{C})+2
$$




$$
\begin{aligned}
& =3-((1 / 2) p(\mathfrak{g}, \mathcal{C})+c(\mathfrak{g}, \mathcal{C})) \\
& =3-(1 / 2)\left(\operatorname{dim}\left(V^{+}\right)+4\right) \\
& =-(1 / 2)\left(\operatorname{dim}\left(V^{+}\right)-2\right)
\end{aligned}
$$

by Proposition 3.1 and so

$$
\begin{aligned}
& {\left[D_{-\gamma}, \Omega_{4}^{\prime}(\mathrm{C})\right]=-4 \xi_{\gamma} \Omega_{4}^{\prime}(\mathrm{C})+2\left(s-s_{4}\right) \sum_{i \in I(\mathcal{C})} \Omega_{1}\left(\left[Z_{i}, X\right]\right) \Omega_{2}\left(Z^{i}\right)} \\
& \quad+(1 / 2)\left(s-s_{2}(\mathcal{C})\right) \operatorname{dim}\left(V^{+}\right) \Lambda(\mathcal{C}) \partial_{\gamma}+\left(s-s_{2}(\mathcal{C})\right) \Lambda(\mathcal{C}) \Omega_{1}(X) \partial_{\gamma} .
\end{aligned}
$$

By combining this evaluation with (6.3) and Lemma 6.14, and simplifying the result, we finally obtain the formula in the statement. We remark that the coefficient of $\partial_{\gamma}^{2}$ in the definition of $\Omega_{4}(\mathcal{C})$ is fixed by the requirement that the $\Omega_{1}(X) \partial_{\gamma}$ term in the above formula for $\left[D_{-\gamma}, \Omega_{4}^{\prime}(\mathcal{C})\right]$ be canceled by the corresponding term in $\left[D_{-\gamma}, \partial_{\gamma}^{2}\right]$.

The construction of $\Omega_{4}$ that has just been given yields zero for the algebra of type $\mathrm{A}_{2}$, since in this case the diagram $\mathcal{D}_{\gamma}(\mathfrak{g}, \mathfrak{h})$ is empty. For the algebra of type $A_{r}$ there is an alternate construction of $\Omega_{4}$ that makes use of the additional central element $Z_{0}$ that was defined at the end of Section 5. As well as defining a non-zero operator $\Omega_{4}$ for the algebra $A_{2}$, it gives a simpler expression than the uniform construction. We finish this section by describing the alternate construction.

Theorem 6.3. $\quad$ Let $\mathfrak{g}$ be the algebra of type $\mathrm{A}_{r}$ and define

$$
\Omega_{4}^{0}=\Omega_{2}\left(Z_{0}\right)^{2}+(1 / 2)\left(s-s_{2}\right) \partial_{\gamma}^{2},
$$

where $s_{2}=(r-1) / 2$. Then

$$
\left[D_{-\gamma}, \Omega_{4}^{0}\right]=-4 \xi_{\gamma} \Omega_{4}^{0}+2\left(s-s_{4}\right) \Omega_{1}\left(\left[Z_{0}, X\right]\right) \Omega_{2}\left(Z_{0}\right)-2\left(s-s_{4}\right)\left(s-s_{2}\right) \partial_{\gamma},
$$

where $s_{4}=(r-2) / 2$.

Proof. We begin by considering $\left[D_{-\gamma}, \Omega_{2}\left(Z_{0}\right)^{2}\right]$. From Theorem 5.3 we have

$$
\left[D_{-\gamma}, \Omega_{2}\left(Z_{0}\right)\right]=-2 \xi_{\gamma} \Omega_{2}\left(Z_{0}\right)+\left(s-s_{2}\right) \Omega_{1}\left(\left[Z_{0}, X\right]\right) .
$$

By using this, we obtain

$$
\begin{aligned}
{\left[D_{-\gamma}, \Omega_{2}\left(Z_{0}\right)^{2}\right]=-4 \xi_{\gamma} \Omega_{2}\left(Z_{0}\right)^{2}+\left(s-s_{2}\right) \Omega_{1}\left(\left[Z_{0}, X\right]\right) \Omega_{2}\left(Z_{0}\right) } \\
+\left(s-s_{2}\right) \Omega_{2}\left(Z_{0}\right) \Omega_{1}\left(\left[Z_{0}, X\right]\right)-2\left[\Omega_{2}\left(Z_{0}\right), \xi_{\gamma}\right] \Omega_{2}\left(Z_{0}\right) .
\end{aligned}
$$


Now

$$
\begin{aligned}
\Omega_{2}\left(Z_{0}\right) \Omega_{1}\left(\left[Z_{0}, X\right]\right)= & \sum_{\alpha} \Omega_{2}\left(Z_{0}\right) \xi_{\alpha} \Omega_{1}\left(\left[Z_{0}, X_{\alpha}\right]\right) \\
= & \sum_{\alpha} \xi_{\alpha} \Omega_{2}\left(Z_{0}\right) \Omega_{1}\left(\left[Z_{0}, X_{\alpha}\right]\right)+\sum_{\alpha}\left[\Omega_{2}\left(Z_{0}\right), \xi_{\alpha}\right] \Omega_{1}\left(\left[Z_{0}, X_{\alpha}\right]\right) \\
= & \Omega_{1}\left(\left[Z_{0}, X\right]\right) \Omega_{2}\left(Z_{0}\right)+\sum_{\alpha} \xi_{\alpha}\left[\Omega_{2}\left(Z_{0}\right), \Omega_{1}\left(\left[Z_{0}, X_{\alpha}\right]\right)\right] \\
& \quad+\sum_{\alpha} N_{\alpha, \alpha^{\prime}}^{-1} \Omega_{1}\left(\left[Z_{0}, X_{\alpha^{\prime}}\right]\right) \Omega_{1}\left(\left[Z_{0}, X_{\alpha}\right]\right) \\
& =\Omega_{1}\left(\left[Z_{0}, X\right]\right) \Omega_{2}\left(Z_{0}\right)+\sum_{\alpha} \xi_{\alpha} \Omega_{1}\left(\left[Z_{0},\left[Z_{0}, X_{\alpha}\right]\right]\right) \partial_{\gamma}+S \\
& =\Omega_{1}\left(\left[Z_{0}, X\right]\right) \Omega_{2}\left(Z_{0}\right)+\Omega_{1}(X) \partial_{\gamma}+S
\end{aligned}
$$

where

$$
S=\sum_{\alpha} N_{\alpha, \alpha^{\prime}}^{-1} \Omega_{1}\left(\left[Z_{0}, X_{\alpha^{\prime}}\right]\right) \Omega_{1}\left(\left[Z_{0}, X_{\alpha}\right]\right)
$$

In this computation, we have used Lemma 6.10 from the second line to the third, Lemma 6.4 from the third line to the fourth, and the identity $\left[Z_{0},\left[Z_{0}, Y\right]\right]=Y$ for all $Y \in V^{+}$from the fourth line to the fifth. The next task is to evaluate $S$, which we do indirectly as follows:

$$
\begin{aligned}
S & =-\sum_{\alpha} N_{\alpha, \alpha^{\prime}}^{-1} \Omega_{1}\left(\left[Z_{0}, X_{\alpha}\right]\right) \Omega_{1}\left(\left[Z_{0}, X_{\alpha^{\prime}}\right]\right) \\
& =-S-\sum_{\alpha} N_{\alpha, \alpha^{\prime}}^{-1}\left[\Omega_{1}\left(\left[Z_{0}, X_{\alpha}\right]\right), \Omega_{1}\left(\left[Z_{0}, X_{\alpha^{\prime}}\right]\right)\right] \\
& =-S-\sum_{\alpha} N_{\alpha, \alpha^{\prime}}^{-1}\left\langle\left[Z_{0}, X_{\alpha}\right],\left[Z_{0}, X_{\alpha^{\prime}}\right]\right\rangle \partial_{\gamma} \\
& =-S+\sum_{\alpha} N_{\alpha, \alpha^{\prime}}^{-1}\left\langle X_{\alpha},\left[Z_{0},\left[Z_{0}, X_{\alpha^{\prime}}\right]\right]\right\rangle \partial_{\gamma} \\
& =-S+\sum_{\alpha} N_{\alpha, \alpha^{\prime}}^{-1}\left\langle X_{\alpha}, X_{\alpha^{\prime}}\right\rangle \partial_{\gamma} \\
& =-S+\sum_{\alpha} \partial_{\gamma} \\
& =-S+\operatorname{dim}\left(V^{+}\right) \partial_{\gamma} .
\end{aligned}
$$

Thus $S=(1 / 2) \operatorname{dim}\left(V^{+}\right) \partial_{\gamma}$. By Lemma 6.10, $\left[\Omega_{2}\left(Z_{0}\right), \xi_{\gamma}\right]=-(1 / 2) \Omega_{1}\left(\left[Z_{0}, X\right]\right)$. These evaluations combine to give

$$
\left[D_{-\gamma}, \Omega_{2}\left(Z_{0}\right)^{2}\right]=-4 \xi_{\gamma} \Omega_{2}\left(Z_{0}\right)^{2}+2\left(s-s_{4}\right) \Omega_{1}\left(\left[Z_{0}, X\right]\right) \Omega_{2}\left(Z_{0}\right)
$$




$$
+\left(s-s_{2}\right) \Omega_{1}(X) \partial_{\gamma}+(1 / 2)\left(s-s_{2}\right) \operatorname{dim}\left(V^{+}\right) \partial_{\gamma} .
$$

According to Lemma 6.14, we have

$$
\left[D_{-\gamma}, \partial_{\gamma}^{2}\right]=-4 \xi_{\gamma} \partial_{\gamma}^{2}-2 \Omega_{1}(X) \partial_{\gamma}-(4 s+2) \partial_{\gamma} .
$$

These two expressions combine to give the required evaluation of $\left[D_{-\gamma}, \Omega_{4}^{0}\right]$. To check this, it is useful to recall that $\operatorname{dim}\left(V^{+}\right)=2(r-1)$.

It appears that we have obtained several operators $\Omega_{4}(\mathcal{C})$ in the cases where $\mathcal{D}_{\gamma}(\mathfrak{g}, \mathfrak{h})$ is not connected, and also when $\mathfrak{g}$ is of type $\mathrm{A}_{r}$ with $r \geq 3$. In fact, the apparently different operators are proportional and so we have obtained essentially only one operator in each case, although we do not prove this here. The proportionality is most easily obtained in the context of the theory of generalized Verma modules and will be considered elsewhere.

\section{$\S 7$. The $b$-Function of the Quasi-Invariant}

In this section we assume that the algebra $\mathfrak{g}$ is not of type $\mathrm{C}_{r}$, so that the relative invariant $\Delta$ on $V^{+}$does not vanish identically. We wish to determine the $b$-function of the quasi-invariant $P \in \mathbb{C}[\mathfrak{n}]$. A first step is to find a polynomial $b(s) \in \mathbb{C}[s]$ such that there is an identity of the form $D P^{s+1}=b(s) P^{s}$ for some differential operator $D$ on $\mathfrak{n}$ whose coefficients are in $\mathbb{C}[s] \otimes_{\mathbb{C}} \mathbb{C}[\mathfrak{n}]$. An identity of this form implies that the $b$-function of $P$ divides $b(s)$. The complete determination of the $b$-function requires additional arguments.

In [8], Gyoja explains how to find a polynomial $b(s)$ having the required property by using a sophisticated algorithm, and carries out the necessary calculations in some cases. We shall instead use a completely elementary approach that expresses a suitable polynomial $b(s)$ directly in terms of the $b$-function of the relative invariant. Since the latter has been computed in all our cases, this will also solve the problem. As well as being elementary, our approach has two advantages. First, it reveals that there is a simple relationship between the roots of the $b$-functions of the relative invariant and the quasi-invariant. Secondly, it yields an explicit differential operator $D$ to accompany the polynomial $b(s)$.

It may happen that there is a relative invariant $\Delta_{0} \in \mathbb{C}\left[V^{+}\right]$associated to the character $\chi$. If so, and $\Delta_{0}$ is normalized so that $\Delta_{0}\left(X_{0}\right)=1$, then $\Delta=\Delta_{0}^{2}$. By inspection of the list of possible prehomogeneous vector spaces $\left(L, \mathrm{Ad}, V^{+}\right)$, one finds that this happens if and only if the algebra $\mathfrak{g}$ is of type $\mathrm{A}_{r}$. For all other types that we are considering, $\Delta$ is irreducible. Our discussion will apply just as well to either case. 
Let $\Delta^{*}$ be the constant coefficient differential operator on $V^{+}$corresponding in the usual way to a relatively invariant polynomial on the dual space of $\left(L, \mathrm{Ad}, V^{+}\right.$) associated to the character $\chi^{-2}$ (see, for example, Proposition 2.22 of [14] and the discussion preceding it). Then there is a quartic polynomial $a(s) \in \mathbb{C}[s]$ such that

$$
\Delta^{*} \Delta^{s+1}=a(s) \Delta^{s} .
$$

As with all similar equations, this equation may be understood to hold on the universal cover of the complement of the singular set in $V^{+}$with $s$ a complex number, in a suitable $\mathcal{D}$-module with $s$ a formal parameter, or on $V^{+}$with $s$ an integer. Note that we do not require the polynomial $a(s)$ to be monic, although this could be arranged by a suitable normalization of $\Delta^{*}$ if desired. The defining equation makes it clear that $a(-1)=0$ and so we may write

$$
a(s)=\left(a_{3} s^{3}+a_{2} s^{2}+a_{1} s+a_{0}\right)(s+1) .
$$

Lemma 7.1. For all $s \in \mathbb{Z}$ we have

$$
\begin{aligned}
& \Delta^{*}\left(y^{2}-\Delta(X)\right)^{s+1}= \\
& \left(A_{0}(s) \Delta^{3}(X)+A_{1}(s) \Delta^{2}(X) y^{2}+A_{2}(s) \Delta(X) y^{4}+A_{3}(s) y^{6}\right)\left(y^{2}-\Delta(X)\right)^{s-3},
\end{aligned}
$$

where

$$
\begin{aligned}
& A_{0}(s)=\left(a_{3} s^{3}+a_{2} s^{2}+a_{1} s+a_{0}\right)(s+1), \\
& A_{1}(s)=-\left(\left(a_{2}+3 a_{3}\right) s^{2}+\left(2 a_{1}+a_{2}-a_{3}\right) s+3 a_{0}\right)(s+1), \\
& A_{2}(s)=\left(\left(a_{1}+a_{2}+a_{3}\right) s+3 a_{0}\right)(s+1), \\
& A_{3}(s)=-a_{0}(s+1) .
\end{aligned}
$$

Proof. We begin by introducing a new variable $z$, which will eventually be identified with $y^{2}$, and letting $L$ act by automorphisms on $\mathbb{C}[z]$ in such a way that $l \cdot z=\chi(l)^{2} z$. Now $\Delta^{*}$ is a constant coefficient differential operator of order four such that $\Delta^{*} z=0$. From this and the product rule it follows that there are polynomials $F_{0}, \ldots, F_{3} \in \mathbb{C}\left[V^{+}\right]$and $A_{0}, \ldots, A_{3} \in \mathbb{C}[s]$ such that

$$
\Delta^{*}(z-\Delta(X))^{s+1}=F(s, X, z)(z-\Delta(X))^{s-3}
$$

with

$$
F(s, X, z)=\left(A_{0}(s) F_{0}(X)+A_{1}(s) F_{1}(X) z+A_{2}(s) F_{2}(X) z^{2}+A_{3}(s) F_{3}(X) z^{3}\right) .
$$

The transformation laws of $\Delta^{*}$ and $(z-\Delta(X))$ under $L$ imply that

$$
F(s, \operatorname{Ad}(l) X, l \cdot z)=\chi(l)^{6} F(s, X, z) .
$$


By setting $z=0$ in this equation, we conclude that $F_{0}$ is a relatively invariant polynomial on $V^{+}$associated to the character $\chi^{6}$ and so $F_{0}$ is proportional to $\Delta^{3}$. We may assume that $F_{0}=\Delta^{3}$ by absorbing the constant of proportionality into $A_{0}$. By considering $\left(F-A_{0} F_{0}\right) / z$, we similarly conclude that $F_{1}$ is proportional to $\Delta^{2}$, and we may assume them equal after changing $A_{1}$ if necessary. Similarly, we may assume that $F_{2}=\Delta$ and $F_{3}=1$. This gives us an identity of the form

$$
\begin{aligned}
& \Delta^{*}(z-\Delta(X))^{s+1}= \\
& \left(A_{0}(s) \Delta^{3}(X)+A_{1}(s) \Delta^{2}(X) z+A_{2}(s) \Delta(X) z^{2}+A_{3}(s) z^{3}\right)(z-\Delta(X))^{s-3} .
\end{aligned}
$$

It remains to identify the polynomials $A_{0}, \ldots, A_{3}$.

By setting $z=0$ on both sides we obtain

$$
(-1)^{s+1} \Delta^{*} \Delta^{s+1}=(-1)^{s-3} A_{0}(s) \Delta^{s}
$$

and, since $s+1$ and $s-3$ are of the same parity, it follows that $A_{0}(s)=a(s)$. Let $\partial_{z}$ denote the partial derivative with respect to $z$ and write $P=z-\Delta(X)$. Then

$$
\partial_{z} P^{s+1}=(s+1) P^{s}
$$

and the operator $\partial_{z}$ commutes with $\Delta^{*}$. We have

$$
\begin{aligned}
\partial_{z} \Delta^{*} P^{s+1} & =\Delta^{*} \partial_{z} P^{s+1} \\
& =(s+1) \Delta^{*} P^{s} \\
& =(s+1)\left(A_{0}(s-1) \Delta^{3}(X)+\cdots+A_{3}(s-1) z^{3}\right) P^{s-4} .
\end{aligned}
$$

On the other hand,

$$
\begin{aligned}
\partial_{z} \Delta^{*} & P^{s+1} \\
= & \partial_{z}\left(\left(A_{0}(s) \Delta^{3}(X)+\cdots+A_{3}(s) z^{3}\right) P^{s-3}\right) \\
= & \left(A_{1}(s) \Delta^{2}(X)+2 A_{2}(s) \Delta(X) z+3 A_{3}(s) z^{2}\right) P^{s-3} \\
& +\left(A_{0}(s) \Delta^{3}(X)+\cdots+A_{3}(s) z^{3}\right)(s-3) P^{s-4} \\
= & \left((s-3) A_{0}(s)-A_{1}(s)\right) \Delta^{3}(X)+\left((s-2) A_{1}(s)-2 A_{2}(s)\right) \Delta^{2}(X) z \\
& \left.+\left((s-1) A_{2}(s)-3 A_{3}(s)\right) \Delta(X) z^{2}+s A_{3}(s) z^{3}\right) P^{s-4} .
\end{aligned}
$$

By comparing coefficients in these two expressions, we obtain the recurrence relations

$$
\begin{aligned}
& A_{1}(s)=(s-3) A_{0}(s)-(s+1) A_{0}(s-1), \\
& 2 A_{2}(s)=(s-2) A_{1}(s)-(s+1) A_{1}(s-1), \\
& 3 A_{3}(s)=(s-1) A_{2}(s)-(s+1) A_{2}(s-1) .
\end{aligned}
$$


A direct calculation using the known value of $A_{0}(s)$ now yields $A_{1}(s), A_{2}(s)$, and $A_{3}(s)$ as in the statement. The proof is completed by replacing $z$ by $y^{2}$.

We are now ready to describe the polynomial $b(s)$. We may factor $a(s)$ as

$$
a(s)=a_{3}(s+1)\left(s-\rho_{1}\right)\left(s-\rho_{2}\right)\left(s-\rho_{3}\right),
$$

and we define $b(s) \in \mathbb{C}[s]$ by

$$
b(s)=a_{3}(s+1)\left(s-\left(\rho_{1}-1 / 2\right)\right)\left(s-\left(\rho_{2}-1 / 2\right)\right)\left(s-\left(\rho_{3}-1 / 2\right)\right) .
$$

A calculation using Newton's identities shows that $b(s)$ may also be expressed in terms of the coefficients $a_{0}, \ldots, a_{3}$ as

$$
\begin{aligned}
& b(s)= \\
& (s+1)\left(a_{3} s^{3}+\left(a_{2}+3 a_{3} / 2\right) s^{2}+\left(a_{1}+a_{2}+3 a_{3} / 4\right) s+\left(a_{0}+a_{1} / 2+a_{2} / 4+a_{3} / 8\right)\right) .
\end{aligned}
$$

In the following, we let $\partial_{y}$ denote the partial derivative with respect to $y$.

Theorem 7.1. With notation as above, define a differential operator on $\mathfrak{n}$ by

$$
D=\Delta^{*}-c(s) \partial_{y}^{2}+r(s) y \partial_{y}^{3}-\left(a_{3} / 16\right) \Delta(X) \partial_{y}^{4}
$$

with

$$
r(s)=\left(a_{3} / 4\right) s+\left(a_{2} / 8+a_{3} / 4\right)
$$

and

$$
c(s)=\left(3 a_{3} / 4\right) s^{2}+\left(3 a_{3} / 4+a_{2} / 2\right) s+\left(a_{1} / 4+a_{2} / 8+a_{3} / 16\right) .
$$

Then we have

$$
D\left(y^{2}-\Delta(X)\right)^{s+1}=-b(s)\left(y^{2}-\Delta(X)\right)^{s} .
$$

Proof. For notational compactness, let us write $\Delta$ for $\Delta(X)$ and $P$ for $y^{2}-\Delta(X)$ throughout the proof. By Lemma 7.1, we have

$$
\Delta^{*} P^{s+1}=\left(A_{0}(s) \Delta^{3}+A_{1}(s) \Delta^{2} y^{2}+A_{2}(s) \Delta y^{4}+A_{3}(s) y^{6}\right) P^{s-3}
$$

with explicit polynomials $A_{0}(s), \ldots, A_{3}(s)$ as given in the lemma. By direct calculation, we also have the identities

$$
\begin{aligned}
& \partial_{y}^{2} P^{s+1} \\
& =-2(s+1)\left(\Delta^{3}-(2 s+3) \Delta^{2} y^{2}+(4 s+3) \Delta y^{4}-(2 s+1) y^{6}\right) P^{s-3} \\
& \partial_{y}^{3} P^{s+1} \\
& =4 s(s+1)\left(3 \Delta^{2} y-2(s+2) \Delta y^{3}+(2 s+1) y^{5}\right) P^{s-3} \\
& \partial_{y}^{4} P^{s+1} \\
& =4 s(s+1)\left(3 \Delta^{2}-6(2 s-1) \Delta y^{2}+\left(4 s^{2}-1\right) y^{4}\right) P^{s-3}
\end{aligned}
$$


Since

$$
-\left(y^{2}-\Delta\right)^{3}=\Delta^{3}-3 \Delta^{2} y^{2}+3 \Delta y^{4}-y^{6},
$$

the identity $D P^{s+1}=-b(s) P^{s}$ amounts to the four polynomial identities

$$
\begin{aligned}
b(s)= & A_{0}(s)+2(s+1) c(s)-\left(3 a_{3} / 4\right) s(s+1), \\
-3 b(s)= & A_{1}(s)-2(s+1)(2 s+3) c(s)+12 s(s+1) r(s) \\
& \quad+\left(3 a_{3} / 2\right) s(s+1)(2 s-1), \\
3 b(s)= & A_{2}(s)+2(s+1)(4 s+3) c(s)-8 s(s+1)(s+2) r(s) \\
& \quad-\left(a_{3} / 4\right) s(s+1)\left(4 s^{2}-1\right), \\
-b(s)= & A_{3}(s)-2(s+1)(2 s+1) c(s)+4 s(s+1)(2 s+1) r(s) .
\end{aligned}
$$

Every quantity appearing in these proposed identities is known explicitly in terms of $a_{0}, \ldots, a_{3}$ and one now verifies by substitution that they do indeed hold. Thus $D P^{s+1}=-b(s) P^{s}$.

Let $D_{0}$ be the differential operator on $\mathfrak{n}$ defined by

$$
D_{0}=6 s \partial_{y}^{2}+2(s-2) y \partial_{y}^{3}-\left(y^{2}-\Delta(X)\right) \partial_{y}^{4} .
$$

One verifies by direct computation that $D_{0}\left(y^{2}-\Delta(X)\right)^{s+1}=0$ for all $s \in \mathbb{Z}$. Thus, for any constant $\lambda \in \mathbb{C}$, the differential operator $D+\lambda D_{0}$ also satisfies $\left(D+\lambda D_{0}\right) P^{s+1}=-b(s) P^{s}$. A particularly convenient choice is $\lambda=a_{3} / 16$, for then the operator $D+\lambda D_{0}$ is the sum of $\Delta^{*}$ and an operator of the form $p_{1}(s) \partial_{y}^{2}+p_{2}(s) y \partial_{y}^{3}-\lambda y^{2} \partial_{y}^{4}$ for certain polynomials $p_{1}(s)$ and $p_{2}(s)$.

As was explained in the introduction to this section, Theorem 7.1 implies that the Bernstein-Sato polynomial $b_{P}(s)$ of the quasi-invariant $P$ divides the polynomial $b(s)$. In fact, it appears to be the case that $b_{P}(s)$ is a constant multiple of $b(s)$ for all the algebras under consideration, but we shall not prove this here. The polynomial $b(s)$ will be referred to as the conjectured BernsteinSato polynomial below.

\section{$\S 8 . \quad$ A Compendium of Cases}

In this section we present a case-by-case summary of data concerning the Heisenberg parabolic in each of the simple Lie algebras. When applicable, we give the extended Dynkin diagram; the highest root $\gamma$; half the sum of the roots in $\mathfrak{n}$, which we denote by $\rho(\mathfrak{n})$; a choice of $\delta \in R\left(V^{+}\right)$; the constants $c(\mathfrak{g}, \mathcal{C})$ and $p(\mathfrak{g}, \mathcal{C})$ that were defined in Section 2 ; the constant $\Lambda(\mathcal{C})$ that was defined in Section 6; a prehomogeneous vector space equivalent to $\left(L, \mathrm{Ad}, V^{+}\right)$; the 
Bernstein-Sato polynomial $b_{\Delta}(s)$ of its relative invariant; and the conjectured Bernstein-Sato polynomial $b(s)$ of the associated quasi-invariant.

Some remarks on notation and sources might be in order. We follow the conventions of [3] for numbering the simple roots. We write $c\left(\mathrm{~A}_{r}\right), c\left(\mathrm{C}_{r}\right)$, and so on for the constants $c(\mathfrak{g}, \mathcal{C})$ in the case where the graph $\mathcal{D}_{\gamma}(\mathfrak{g}, \mathfrak{h})$ is connected. In the cases where it is not, we let $\mathcal{C}_{1}$ be the component containing $\alpha_{1}$ and $\mathcal{C}_{2}$ be the component containing $\alpha_{r}$. For $\mathrm{D}_{4}$, there is a third component $\mathcal{C}_{3}$ consisting of $\alpha_{3}$ alone. Except for types $\mathrm{A}_{r}, \mathrm{~B}_{3}$ and $\mathrm{C}_{r}$, the prehomogeneous vector space $\left(L, \mathrm{Ad}, V^{+}\right)$is irreducible, reduced and regular, and so the $b$ function can be found in the invaluable reference [13]. The remaining data is easily verified. We say that prehomogeneous vector spaces $\left(L_{1}, \pi_{1}, V_{1}\right)$ and $\left(L_{2}, \pi_{2}, V_{2}\right)$ are equivalent if there is a linear isomorphism $\theta: V_{1} \rightarrow V_{2}$ such that $\pi_{2}\left(L_{2}\right)=\theta \circ \pi_{1}\left(L_{1}\right) \circ \theta^{-1}$. Equivalent prehomogeneous vector spaces have the same $b$-function.

§8.1. $\quad \mathrm{A}_{r}, r \geq 2$

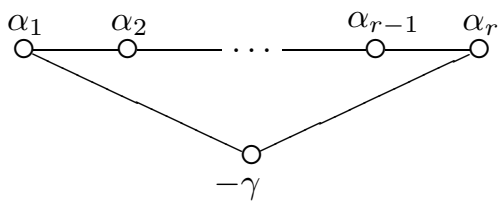

The highest root is $\gamma=\alpha_{1}+\cdots+\alpha_{r}, \rho(\mathfrak{n})=(r / 2) \gamma$ and we may take $\delta=\alpha_{1}$. We have $c\left(\mathrm{~A}_{r}\right)=2$ and $p\left(\mathrm{~A}_{r}\right)=2(r-1)$ provided that $r \geq 3$; if $r=2$ then $\mathcal{D}_{\gamma}(\mathfrak{g}, \mathfrak{h})$ is empty and so $c\left(\mathrm{~A}_{2}\right)$ and $p\left(\mathrm{~A}_{2}\right)$ are undefined. A space equivalent to $\left(L, \mathrm{Ad}, V^{+}\right)$is given by $\mathrm{GL}(1) \times \mathrm{GL}(r-1)$ acting on $\mathbb{C}^{r-1} \oplus \mathbb{C}^{r-1}$ via

$$
(\lambda, g)\left(v_{1}, v_{2}\right)=\left(\lambda \operatorname{det}(g) g v_{1}, \lambda^{t} g^{-1} v_{2}\right) .
$$

The relative invariant $\Delta_{0}\left(v_{1}, v_{2}\right)={ }^{t} v_{1} v_{2}$ is associated to the character $\chi_{0}(\lambda, g)=$ $\lambda^{2} \operatorname{det}(g)$. Its $b$-function is $a_{0}(s)=(s+1)(s+(r-1))$. The $b$-function of $\Delta=\Delta_{0}^{2}$ is

$$
b_{\Delta}(s)=(s+1)(s+1 / 2)(s+(r-1) / 2)(s+r / 2)
$$

and the conjectured Bernstein-Sato polynomial of the quasi-invariant is

$$
b(s)=(s+1)^{2}(s+r / 2)(s+(r+1) / 2) .
$$


§8.2. $\quad \mathrm{B}_{r}, r \geq 3$

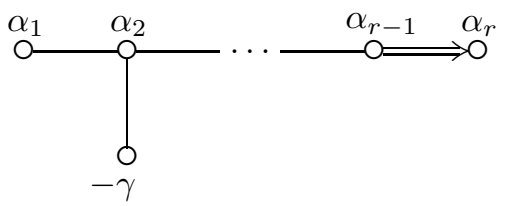

The highest root is $\gamma=\alpha_{1}+2 \alpha_{2}+\cdots+2 \alpha_{r}, \rho(\mathfrak{n})=(r-1) \gamma$ and we may take $\delta=\alpha_{2}$. We have $c\left(\mathrm{~B}_{r}, \mathfrak{C}_{1}\right)=2 r-3, p\left(\mathrm{~B}_{r}, \mathfrak{C}_{1}\right)=4, \Lambda\left(\mathfrak{C}_{1}\right)=2 r-7 / 2$, $c\left(\mathrm{~B}_{r}, \mathrm{C}_{2}\right)=4, p\left(\mathrm{~B}_{r}, \mathrm{C}_{2}\right)=2(2 r-5)$, and $\Lambda\left(\mathrm{C}_{2}\right)=2 r-3$. A space equivalent to $\left(L, \mathrm{Ad}, V^{+}\right)$is given by $\mathrm{GL}(2) \times \mathrm{SO}(2 r-3)$ acting naturally on the tensor product $\mathbb{C}^{2} \otimes \mathbb{C}^{2 r-3}$, which we may identify with the space of ordered pairs of vectors in $\mathbb{C}^{m}$ with $m=2 r-3$. The $b$-function of $\Delta$ is

$$
b_{\Delta}(s)=(s+1)(s+3 / 2)(s+(r-2))(s+(r-3 / 2))
$$

and the conjectured Bernstein-Sato polynomial of the quasi-invariant is

$$
b(s)=(s+1)(s+2)(s+(r-3 / 2))(s+(r-1)) .
$$

$\S 8.3 . \quad \mathrm{C}_{r}, r \geq 2$

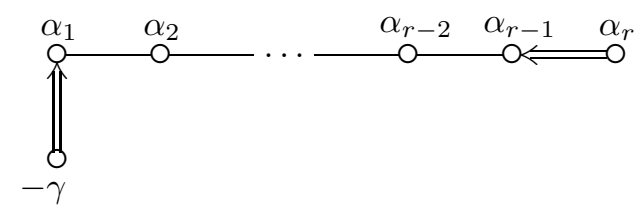

The highest root is $\gamma=2 \alpha_{1}+\cdots+2 \alpha_{r-1}+\alpha_{r}, \rho(\mathfrak{n})=(r / 2) \gamma$ and no choice of $\delta$ is possible. We have $c\left(\mathrm{C}_{r}\right)=1$. A space equivalent to $\left(L, \mathrm{Ad}, V^{+}\right)$ is given by $\mathrm{GL}(1) \times \mathrm{Sp}(2 r-2)$ acting naturally on $\mathbb{C}^{2 r-2}$. There is no relative invariant. The Bernstein-Sato polynomial of the quasi-invariant is

$$
b_{P}(s)=(s+1)(s+1 / 2) \text {. }
$$

§8.4. $\mathrm{D}_{r}, r \geq 4$

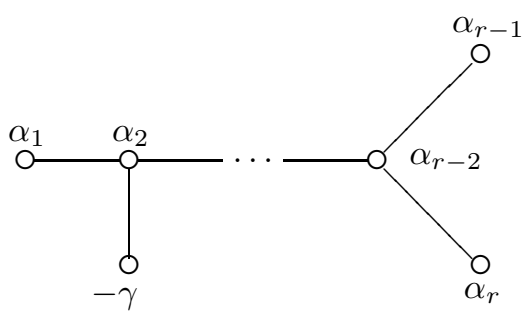


The highest root is $\gamma=\alpha_{1}+2 \alpha_{2}+\cdots+2 \alpha_{r-2}+\alpha_{r-1}+\alpha_{r}, \rho(\mathfrak{n})=$ $(r-3 / 2) \gamma$ and we may take $\delta=\alpha_{2}$. When $r \geq 5$, we have $c\left(\mathrm{D}_{r}, \mathrm{C}_{1}\right)=2 r-4$, $p\left(\mathrm{D}_{r}, \mathrm{C}_{1}\right)=4, \Lambda\left(\mathrm{C}_{1}\right)=2 r-9 / 2, c\left(\mathrm{D}_{r}, \mathrm{C}_{2}\right)=4, p\left(\mathrm{D}_{r}, \mathrm{C}_{2}\right)=4(r-3)$, and $\Lambda\left(\mathrm{C}_{2}\right)=2 r-4$. When $r=4$, we have $c\left(\mathrm{D}_{4}, \mathrm{C}_{i}\right)=p\left(\mathrm{D}_{4}, \mathrm{C}_{i}\right)=4$ and $\Lambda\left(\mathrm{C}_{i}\right)=7 / 2$ for $1 \leq i \leq 3$. A space equivalent to $\left(L, \mathrm{Ad}, V^{+}\right)$is given by $\mathrm{GL}(2) \times \mathrm{SO}(2 r-4)$ acting naturally on the tensor product $\mathbb{C}^{2} \otimes \mathbb{C}^{2 r-4}$, which we may identify with the space of ordered pairs of vectors in $\mathbb{C}^{m}$ with $m=2 r-4$. The $b$-function of $\Delta$ is

$$
b_{\Delta}(s)=(s+1)(s+3 / 2)(s+(r-5 / 2))(s+(r-2))
$$

and the conjectured Bernstein-Sato polynomial of the quasi-invariant is

$$
b(s)=(s+1)(s+2)(s+(r-2))(s+(r-3 / 2)) .
$$

$\S 8.5 . \quad \mathrm{E}_{6}$

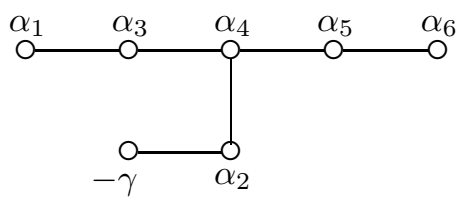

The highest root is $\gamma=\alpha_{1}+2 \alpha_{2}+2 \alpha_{3}+3 \alpha_{4}+2 \alpha_{5}+\alpha_{6}, \rho(\mathfrak{n})=(11 / 2) \gamma$ and we may take $\delta=\alpha_{2}$. We have $c\left(\mathrm{E}_{6}\right)=6, p\left(\mathrm{E}_{6}\right)=12$, and $\Lambda\left(\mathrm{E}_{6}\right)=21 / 2$. A space equivalent to $\left(L, \mathrm{Ad}, V^{+}\right)$is given by $\mathrm{GL}(6)$ acting naturally on $\wedge^{3} \mathbb{C}^{6}$. The $b$-function of $\Delta$ is

$$
b_{\Delta}(s)=(s+1)(s+5 / 2)(s+7 / 2)(s+5)
$$

and the conjectured Bernstein-Sato polynomial of the quasi-invariant is

$$
b(s)=(s+1)(s+3)(s+4)(s+11 / 2) \text {. }
$$

§8.6. $\mathrm{E}_{7}$

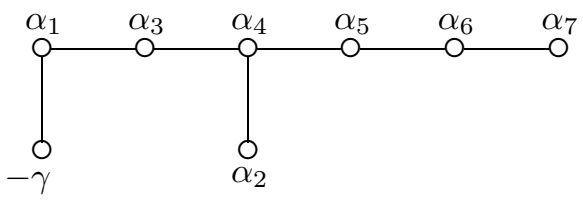


The highest root is $\gamma=2 \alpha_{1}+2 \alpha_{2}+3 \alpha_{3}+4 \alpha_{4}+3 \alpha_{5}+2 \alpha_{6}+\alpha_{7}, \rho(\mathfrak{n})=$ $(17 / 2) \gamma$ and we may take $\delta=\alpha_{1}$. We have $c\left(\mathrm{E}_{7}\right)=8, p\left(\mathrm{E}_{7}\right)=20$, and $\Lambda\left(\mathrm{E}_{7}\right)=33 / 2$. A space equivalent to $\left(L, \mathrm{Ad}, V^{+}\right)$is obtained by taking $\mathrm{GL}(1) \times$ Spin(12) acting on either of its half-spin representations (which are equivalent as prehomogeneous vector spaces, although not, of course, as representations). The $b$-function of $\Delta$ is

$$
b_{\Delta}(s)=(s+1)(s+7 / 2)(s+11 / 2)(s+8)
$$

and the conjectured Bernstein-Sato polynomial of the quasi-invariant is

$$
b(s)=(s+1)(s+4)(s+6)(s+17 / 2) .
$$

$\S 8.7 . \quad \mathrm{E}_{8}$

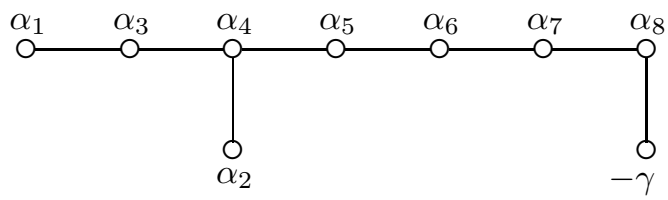

The highest root is $\gamma=2 \alpha_{1}+3 \alpha_{2}+4 \alpha_{3}+6 \alpha_{4}+5 \alpha_{5}+4 \alpha_{6}+3 \alpha_{7}+2 \alpha_{8}$, $\rho(\mathfrak{n})=(29 / 2) \gamma$ and we may take $\delta=\alpha_{8}$. We have $c\left(\mathrm{E}_{8}\right)=12, p\left(\mathrm{E}_{8}\right)=36$, and $\Lambda\left(\mathrm{E}_{8}\right)=57 / 2$. A space equivalent to $\left(L, \mathrm{Ad}, V^{+}\right)$is obtained by taking $\mathrm{GL}(1) \times \mathrm{E}_{7}$ acting on its 56-dimensional irreducible representation. The $b$ function of $\Delta$ is

$$
b_{\Delta}(s)=(s+1)(s+11 / 2)(s+19 / 2)(s+14)
$$

and the conjectured Bernstein-Sato polynomial of the quasi-invariant is

$$
b(s)=(s+1)(s+6)(s+10)(s+29 / 2) .
$$

§8.8. $\mathrm{F}_{4}$

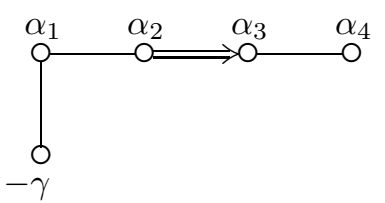

The highest root is $\gamma=2 \alpha_{1}+3 \alpha_{2}+4 \alpha_{3}+2 \alpha_{4}, \rho(\mathfrak{n})=4 \gamma$ and we may take $\delta=\alpha_{1}$. We have $c\left(\mathrm{~F}_{4}\right)=5, p\left(\mathrm{~F}_{4}\right)=8$, and $\Lambda\left(\mathrm{F}_{4}\right)=15 / 2$. A space equivalent 
to $\left(L, \mathrm{Ad}, V^{+}\right)$is given by $\mathrm{GL}(1) \times \mathrm{Sp}(6)$ acting naturally on the 14 -dimensional subspace of $\wedge^{3} \mathbb{C}^{6}$ consisting of primitive forms. The $b$-function of $\Delta$ is

$$
b_{\Delta}(s)=(s+1)(s+2)(s+5 / 2)(s+7 / 2)
$$

and the conjectured Bernstein-Sato polynomial of the quasi-invariant is

$$
b(s)=(s+1)(s+5 / 2)(s+3)(s+4) .
$$

$\S 8.9 . \quad \mathrm{G}_{2}$

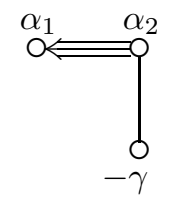

The highest root is $\gamma=3 \alpha_{1}+2 \alpha_{2}, \rho(\mathfrak{n})=(3 / 2) \gamma$ and we may take $\delta=\alpha_{2}$. We have $c\left(\mathrm{G}_{2}\right)=10 / 3, p\left(\mathrm{G}_{2}\right)=4 / 3$, and $\Lambda\left(\mathrm{G}_{2}\right)=5 / 2$. A space equivalent to $\left(L, \mathrm{Ad}, V^{+}\right)$is given by GL(2) acting naturally on $\vee^{3} \mathbb{C}^{2}$. The $b$-function of $\Delta$ is

$$
b_{\Delta}(s)=(s+1)^{2}(s+5 / 6)(s+7 / 6)
$$

and the Bernstein-Sato polynomial of the quasi-invariant is

$$
b_{P}(s)=(s+1)(s+3 / 2)(s+4 / 3)(s+5 / 3) .
$$

\section{$\S 8.10$. The Special Values of $s$}

In the following table, we display the special values of the parameter $s$ for each of the conformally invariant systems that we have constructed. For types other than $\mathrm{A}_{r}$, we use $\Omega_{2}^{\text {big }}$ to stand for the $\Omega_{2}$ system associated to the larger component of $\mathcal{D}_{\gamma}(\mathfrak{g}, \mathfrak{h})$, and $\Omega_{2}^{\text {small }}$ to stand for that associated to the singleton component. For type $\mathrm{A}_{r}$, we use $\Omega_{2}^{\mathrm{big}}$ to stand for the $\Omega_{2}$ system constructed by the normal means, and $\Omega_{2}^{\text {small }}$ to stand for $\Omega_{2}\left(Z_{0}\right)$. A dash indicates that no such system exists in that type. A question mark indicates that the existence is presently unknown; the authors hope to resolve this uncertainty in future work. 


\begin{tabular}{cccccc} 
Type & $\Omega_{1}$ & $\Omega_{2}^{\text {big }}$ & $\Omega_{2}^{\text {small }}$ & $\Omega_{3}$ & $\Omega_{4}$ \\
\hline $\mathrm{A}_{r}(r \geq 2)$ & 0 & 0 & $\frac{r-1}{2}$ & $?$ & $\frac{r-2}{2}$ \\
$\mathrm{~B}_{r}(r \geq 3)$ & 0 & $r-\frac{5}{2}$ & 1 & $?$ & $r-2$ \\
$\mathrm{C}_{r}(r \geq 2)$ & 0 & $-\frac{1}{2}$ & - & - & - \\
$\mathrm{D}_{r}(r \geq 5)$ & 0 & $r-3$ & 1 & $?$ & $r-\frac{5}{2}$ \\
$\mathrm{E}_{6}$ & 0 & 2 & - & 3 & $\frac{9}{2}$ \\
$\mathrm{E}_{7}$ & 0 & 3 & - & 5 & $\frac{15}{2}$ \\
$\mathrm{E}_{8}$ & 0 & 5 & - & 9 & $\frac{27}{2}$ \\
$\mathrm{~F}_{4}$ & 0 & $\frac{3}{2}$ & - & 2 & 3 \\
$\mathrm{G}_{2}$ & 0 & $\frac{2}{3}$ & - & $\frac{1}{3}$ & $\frac{1}{2}$ \\
\hline
\end{tabular}

In type $\mathrm{A}_{2}$, the system $\Omega_{2}^{\text {big }}$ is zero. Type $\mathrm{D}_{4}$ is excluded from the above table, since in this case $\mathcal{D}_{\gamma}(\mathfrak{g}, \mathfrak{h})$ has three singleton components, each with an associated $\Omega_{2}$ system. The special $s$ values for $\mathrm{D}_{4}$ are as follows.

\begin{tabular}{ccccccc} 
Type & $\Omega_{1}$ & $\Omega_{2(1)}$ & $\Omega_{2(2)}$ & $\Omega_{2(3)}$ & $\Omega_{3}$ & $\Omega_{4}$ \\
\hline $\mathrm{D}_{4}$ & 0 & 1 & 1 & 1 & $?$ & $\frac{3}{2}$ \\
\hline
\end{tabular}

\section{References}

[1] A. Astashkevich and R. Brylinski, Exotic differential operators on complex minimal nilpotent orbits, in Advances in geometry, 19-51, Progr. Math., 172, Birkhäuser, Boston, Boston, MA, 1999.

[2] L. Barchini, A. C. Kable and R. Zierau, Conformally invariant systems of differential operators, Preprint 2007.

[3] N. Bourbaki, Lie groups and Lie algebras. Chapters 4-6, Translated from the 1968 French original by Andrew Pressley, Springer, Berlin, 2002.

[4] Lie groups and Lie algebras. Chapters 7-9, Translated from the 1975 and 1982 French originals by Andrew Pressley, Springer, Berlin, 2005.

[5] R. W. Carter, Simple groups of Lie type, Reprint of the 1972 original, Wiley, New York, 1989.

[6] D. H. Collingwood and B. Shelton, A duality theorem for extensions of induced highest weight modules, Pacific J. Math. 146 (1990), no. 2, 227-237.

[7] M. G. Davidson, T. J. Enright and R. J. Stanke, Differential operators and highest weight representations, Mem. Amer. Math. Soc. 94 (1991), no. 455, iv+102 pp.

[8] A. Gyoja, Highest weight modules and $b$-functions of semi-invariants, Publ. Res. Inst. Math. Sci. 30 (1994), no. 3, 353-400. 
[9] R. Howe, Perspectives on invariant theory: Schur duality, multiplicity-free actions and beyond, in The Schur lectures (1992) (Tel Aviv), 1-182, Bar-Ilan Univ., Ramat Gan, 1995.

[10] J.-S. Huang, Intertwining differential operators and reducibility of generalized Verma modules, Math. Ann. 297 (1993), no. 2, 309-324.

[11] H. P. Jakobsen, Basic covariant differential operators on Hermitian symmetric spaces, Ann. Sci. École Norm. Sup. (4) 18 (1985), no. 3, 421-436.

[12] J. C. Jantzen, Kontravariante Formen auf induzierten Darstellungen halbeinfacher LieAlgebren, Math. Ann. 226 (1977), no. 1, 53-65.

[13] T. Kimura, The $b$-functions and holonomy diagrams of irreducible regular prehomogeneous vector spaces, Nagoya Math. J. 85 (1982), 1-80.

[14] _ Introduction to prehomogeneous vector spaces, Translated from the 1998 Japanese original by Makoto Nagura and Tsuyoshi Niitani and revised by the author, Amer. Math. Soc., Providence, RI, 2003.

[15] A. W. Knapp, Lie groups beyond an introduction, Progr. Math., 140, Birkhäuser Boston, Boston, MA, 1996.

[16] B. Kostant, Verma modules and the existence of quasi-invariant differential operators, in Non-commutative harmonic analysis (Actes Colloq., Marseille-Luminy, 1974), 101-128. Lecture Notes in Math., 466, Springer, Berlin.

[17] H. Rubenthaler, Espaces vectoriels préhomogènes, sous-groupes paraboliques et $\mathfrak{s l}_{2}$ triplets, C. R. Acad. Sci. Paris Sér. A-B 290 (1980), no. 3, A127-A129.

[18] _ Algèbres de Lie et Espaces Préhomogènes, Travaux en Cours, 44, Hermann, Paris, 1992. 